\title{
The Dawes Review 3: The Atmospheres of Extrasolar Planets and Brown Dwarfs
}

\author{
Jeremy Bailey ${ }^{1,2}$ \\ ${ }^{1}$ School of Physics, University of New South Wales, NSW, 2052, Australia \\ ${ }^{2}$ Email: j.bailey@unsw.edu.au
}

(ReCEIVED January 10, 2014; AcCePTED September 20, 2014)

\begin{abstract}
The last few years has seen a dramatic increase in the number of exoplanets known and in the range of methods for characterising their atmospheric properties. At the same time, new discoveries of increasingly cooler brown dwarfs have pushed down their temperature range which now extends down to Y-dwarfs of $<300 \mathrm{~K}$. Modelling of these atmospheres has required the development of new techniques to deal with the molecular chemistry and clouds in these objects. The atmospheres of brown dwarfs are relatively well understood, but some problems remain, in particular the behavior of clouds at the L/T transition. Observational data for exoplanet atmosphere characterisation is largely limited to giant exoplanets that are hot because they are near to their star (hot Jupiters) or because they are young and still cooling. For these planets there is good evidence for the presence of $\mathrm{CO}$ and $\mathrm{H}_{2} \mathrm{O}$ absorptions in the IR. Sodium absorption is observed in a number of objects. Reflected light measurements show that some giant exoplanets are very dark, indicating a cloud free atmosphere. However, there is also good evidence for clouds and haze in some other planets. It is also well established that some highly irradiated planets have inflated radii, though the mechanism for this inflation is not yet clear. Some other issues in the composition and structure of giant exoplanet atmospheres such as the occurrence of inverted temperature structures, the presence or absence of $\mathrm{CO}_{2}$ and $\mathrm{CH}_{4}$, and the occurrence of high $\mathrm{C} / \mathrm{O}$ ratios are still the subject of investigation and debate.
\end{abstract}

Keywords: planets and satellites: atmospheres - brown dwarfs - planetary systems - techniques: spectroscopic

The Dawes Reviews are substantial reviews of topical areas in astronomy, published by authors of international standing at the invitation of the PASA Editorial Board. The reviews recognise William Dawes (1762-1836), second lieutenant in the Royal Marines and the astronomer on the First Fleet. Dawes was not only an accomplished astronomer, but spoke five languages, had a keen interest in botany, mineralogy, engineering, cartography and music, compiled the first Aboriginal-English dictionary, and was an outspoken opponent of slavery.

\section{INTRODUCTION}

It is appropriate to consider the properties of extrasolar planet and brown dwarf atmospheres together because they have many similarities. Planets and brown dwarfs cover similar temperature ranges and have similar radii. Planets extend up from very low temperatures (such as those of the ice giants Uranus and Neptune in our Solar system) to effective temperatures of $\sim 3000 \mathrm{~K}$ in hot Jupiters, while new discoveries are continually pushing down the temperature of the coolest known brown dwarfs. The recently discovered Y dwarf class have temperatures as low as $\sim 300 \mathrm{~K}$ (Cushing et al. 2011).

The important processes that occur in these atmospheres are also similar as these are determined primarily by effective temperature. Molecules, chemistry and clouds are important in determining the opacities and hence structure of all these objects. At any temperature below about $\sim 2000 \mathrm{~K}$, solid and liquid condensates can start to form, resulting in considerable complications compared with higher temperatures where only gas phase processes need to be considered. More complex molecules such as methane $\left(\mathrm{CH}_{4}\right)$ become important and the excitation of high vibrational and rotational levels mean that vast numbers of spectral lines are needed to model the opacity. The modelling of these atmospheres thus presents new challenges compared with those encountered in conventional stellar atmosphere models, and these challenges are largely common to the modeling of both exoplanets and brown dwarfs. The differences between giant exoplanets and brown dwarfs include the generally lower mass (and hence gravity) in exoplanets, and the difference in environment. An exoplanet orbits a star, and the stellar illumination can have a 
significant influence on its structure and properties, particularly for close in planets such as hot Jupiters. The presence of the host star also impacts on our ability to observe the planet. While some observations can be easier for planets than brown dwarfs (e.g. determining mass and radius), spectroscopy to characterise the atmospheres is usually extremely challenging for exoplanets while relatively straightforward for brown dwarfs.

The structure of this review will be to begin with looking at brown dwarf atmospheres. This reflects the fact that these are better observed and understood objects, without the complications that are introduced by the presence of the host star in exoplanet systems, but nevertheless illustrate many of the processes that are also important in giant exoplanets. Exoplanet atmospheres will be considered next, with a brief look at the giant planets in our own solar system as a guide. Observations relevant to atmospheric structure and composition are now being obtained by a number of methods primarily for giant exoplanets. These will be outlined and the results of these methods discussed.

The next section will look at the modelling of brown dwarf and exoplanet atmospheres. The techniques are very similar for both classes of objects. A final section will look at the possibilities of detecting extrasolar habitable planets and searching for signatures of life on such planets.

\section{BROWN DWARFS}

\subsection{History and properties}

The existence of brown dwarfs was predicted long before they were recognised observationally. Kumar (1963) and Hayashi \& Nakano (1963) showed that there was a lower limit to the mass of a main-sequence star below which hydrogen burning could not occur. Kumar referred to the objects below this limit as 'black' dwarfs, but the name 'brown dwarf' proposed by Tarter (1975) is the one that has been adopted. More recent models set the hydrogen burning mass limit at 0.072 $0.075 \mathrm{M}_{\odot}$ for a solar composition and somewhat higher for lower metallicities (Chabrier \& Baraffe 1997; Burrows et al. 2001).

Because brown dwarfs do not have a continuing nuclear energy source, their evolution is a process of cooling and decreasing luminosity with age. Unlike stars on the main sequence whose properties are determined primarily by their mass, the temperature and luminosity of a brown dwarf are determined by both mass and age. This is illustrated in Figure 1 where the effective temperatures of low mass objects with masses between 0.0005 and $0.1 \mathrm{M}_{\odot}$ are plotted as a function of age. The evolutionary models used here are those of Baraffe et al. (2003) but similar general trends would be obtained with other recent models (e.g Chabrier et al. 2000a; Burrows et al. 2001; Baraffe et al. 2002; Saumon \& Marley 2008).

The four tracks at the top of Figure 1 are for objects massive enough to be stars, so their effective temperature even-

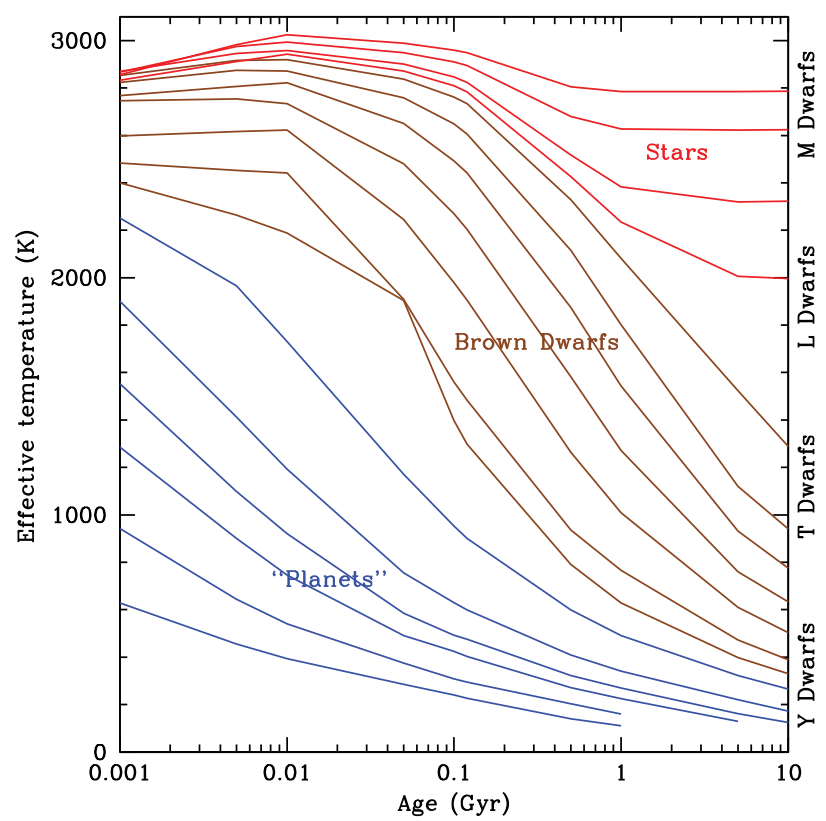

Figure 1. Evolution of effective temperature for objects from 0.0005 to $0.1 \mathrm{M}_{\odot}$ based on the models of Baraffe et al. (2003). The red tracks are for stars with masses above the hydrogen burning limit. The magenta tracks are for brown dwarfs, and the blue tracks are for objects below the deuterium burning limit (planets or sub brown dwarfs) The tracks plotted from top to bottom are masses of (Stars: 0.1, 0.09, 0.08, 0.075 $\mathrm{M}_{\odot}$ ) (Brown Dwarfs: $0.07,0.06,0.05,0.04,0.03,0.02,0.015 \mathrm{M}_{\odot}$ ), (Planets: 0.01, 0.005, 0.003, $0.002,0.001,0.0005 \mathrm{M}_{\odot}$ ).

tually stabilises to a near constant value. However, for brown dwarfs the effective temperature continues to decrease with increasing age. It can be seen from this diagram that a determination of effective temperature alone is not sufficient to determine whether an object is a star or a brown dwarf. An object with $\mathrm{T}_{e f f}=2200 \mathrm{~K}$, for example, can be a young brown dwarf or an older star.

This age-mass degeneracy complicated the early observational search for brown dwarfs, and while several candidates were found (e.g. GD165b Becklin \& Zuckerman 1988) it was not possible to confirm them as brown dwarfs. That changed in 1995 with the discovery of Gl 229b (Nakajima et al. 1995; Oppenheimer et al. 1995), an object sufficiently cool to be unambiguosly a brown dwarf, and with the use of the lithium test to confirm the brown dwarf nature of several objects in the Pleiades cluster (Rebolo, Zapatero-Osorio, \& Martin 1995; Rebolo et al. 1996; Basri, Marcy, \& Graham 1996). The lithium test (Rebolo, Martín, \& Magazzu 1992) relies on the fact that lithium is destroyed by nuclear reactions down to masses just below the hydrogen burning limit. Since cool dwarfs are fully convective, lithium would be removed from the photosphere if these reactions occurred. Hence the presence of lithium in the spectrum can be used to confirm that an object is a brown dwarf.

The deuterium burning mass limit which is at about $13 \mathrm{M}_{J}$ or $0.0125 \mathrm{M}_{\odot}$ (Saumon et al. 1996; Burrows et al. 1997; Chabrier et al. 2000b) is usually considered to be the lower mass limit for brown dwarfs. Objects below this mass limit 


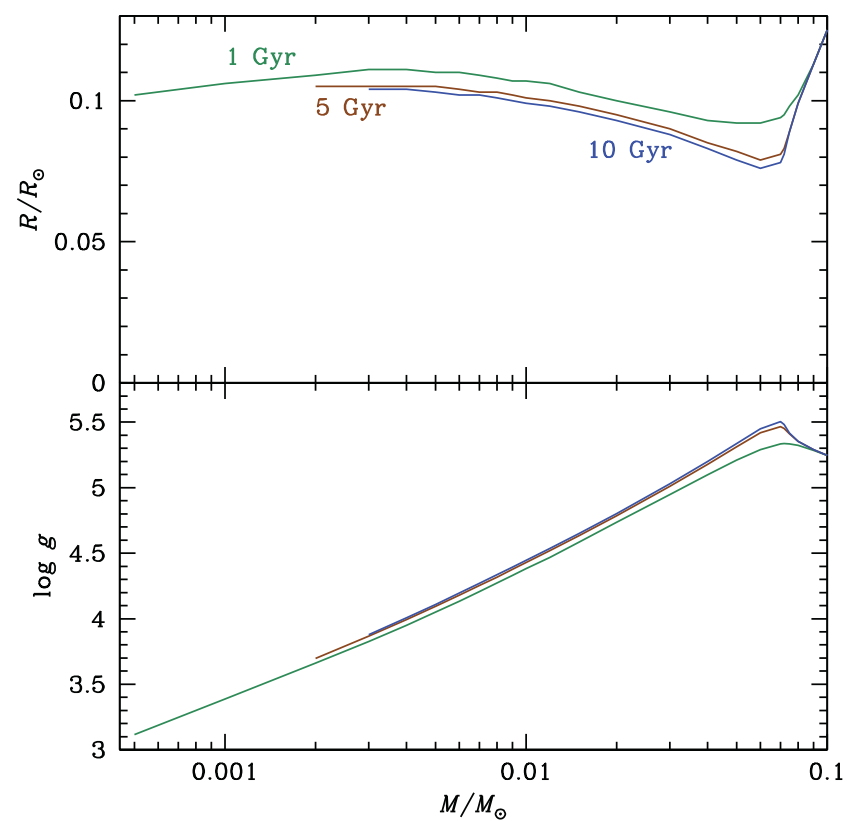

Figure 2. Radius and surface gravity (log g in cgs units) as function of mass for the models of Baraffe et al. (2003) at ages of $1 \mathrm{Gyr}, 5 \mathrm{GYr}$ and $10 \mathrm{Gyr}$.

that orbit stars are generally agreed to be designated as planets. There is less consensus on how to refer to object below this mass limit that do not orbit a star. While these are sometimes referred to as 'free-floating planets' (Lucas \& Roche 2000; Delorme et al. 2012) it has also been argued that such objects should not be referred to as planets but as 'sub-brown dwarfs' or some other designation (see Boss et al. 2003; Basri \& Brown 2006, for a discussion of the issues involved in this controversy).

The electron degeneracy in the cores of brown dwarfs results in their radius varying little with mass as can be seen in Figure 2. All brown dwarfs (except at very young ages) have radii not far from $0.1 \mathrm{R}_{\odot}$ or about 1 Jupiter radius. A consequence of this is that surface gravity $\left(g=G M / R^{2}\right)$ varies with mass from more than $1000 \mathrm{~m} \mathrm{~s}^{-2}(\log g=5$ in cgs units) to around $30 \mathrm{~m} \mathrm{~s}^{-2}$ for Jupiter mass objects as shown in the lower panel of Figure 2.

Brown dwarfs are objects whose atmospheric composition is dominated by molecular gas, as opposed to atoms and ions in the case of hotter stars. This is apparent from Figure 3 which shows the chemical equilibrium composition of a solar composition gas (using the abundances of Grevesse, Asplund $\&$ Sauval 2007). It shows the division of the material by mass fraction into ions, atoms, gas-phase molecules and solid or liquid condensates as calculated by the chemical model of Bailey \& Kedziora-Chudczer (2012). It can be seen that molecules become dominant over atoms for temperatures below about $3500 \mathrm{~K}$. Helium and other noble gases persist as atoms at all temperatures, but other elements are mostly in the form of molecules. Below about $2000 \mathrm{~K}$ condensed phases start to appear, and become a significant fraction of the material. At lower pressures, as shown in the lower panel, the pattern is similar but shifted to lower temperatures.

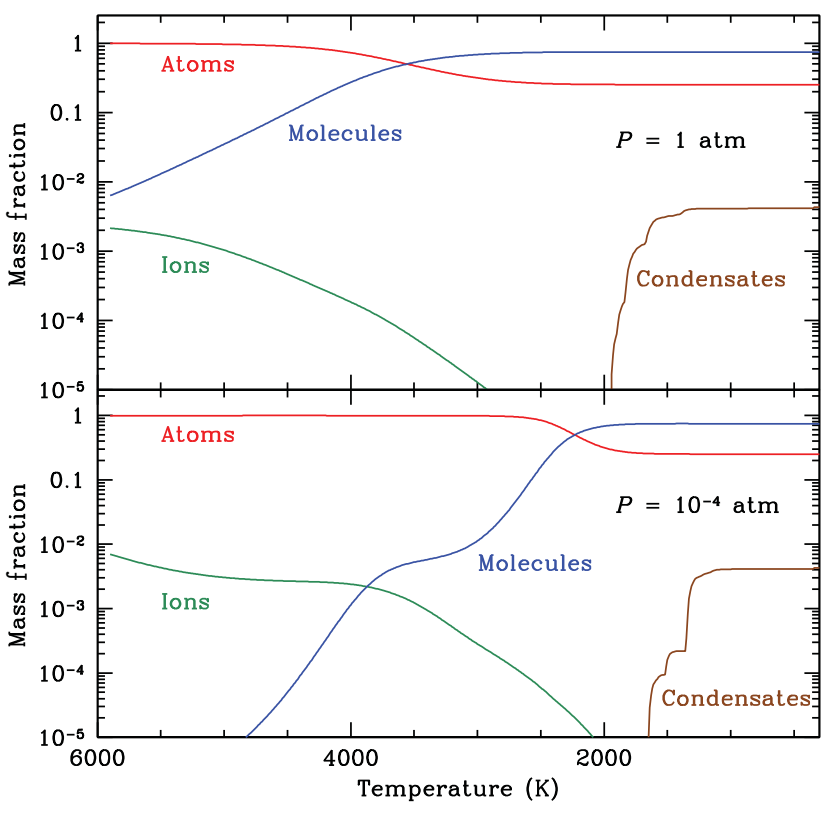

Figure 3. Equilibrium composition of a gas with solar elemental abundances as a function of temperature at two different pressures using the chemical model of Bailey \& Kedziora-Chudczer (2012).

The number of ultracool dwarfs ${ }^{1}$ has increased rapidly over the years since the recognition of the first brown dwarfs in 1995. Most of the objects have come from deep surveys such as the Sloan Digital Sky Survey (SDSS - Fan et al 2000; Hawley et al. 2002) and the Canada-France Brown Dwarfs Survey (CFBDS - Delorme et al. 2008a; Albert et al. 2011) and particularly from infrared surveys such as the Deep Near-Infrared Sky Survey (DENIS - Delfosse et al. 1997; Martín, Delfosse, \& Guieu 2004), the 2 Micron All Sky Survey (2MASS - Kirkpatrick et al. 2000; Burgasser et al. 2002, 2004), and the UKIRT Infrared Deep Sky Survey (UKIDSS - Pinfield et al. 2008; Burningham et al. 2010, 2013).

The most recent additions have come from the Wide-field Infrared Survey Explorer (WISE - Wright et al. 2010). This Earth orbiting NASA mission surveyed the entire sky at four wavelengths $(3.4,4.6,12$ and $22 \mu \mathrm{m})$. The first of these wavelengths probes a deep $\mathrm{CH}_{4}$ absorption band in brown dwarfs. WISE has proved effective in identifying the coolest brown dwarfs. It has led to the discovery of many $\mathrm{T}$ dwarfs (Kirkpatrick et al. 2011; Mace et al. 2013) and to the first Y dwarfs (Cushing et al. 2011; Kirkpatrick et al. 2012; Tinney et al. 2012).

Other recent discoveries from WISE are that of a binary brown dwarf (Luhman 2013) and an extremely cool brown dwarf (Luhman 2014) both at distances of around 2pc. WISE J104915.57-531906.1 (also known as Luhman 16) consists of an L7.5-L8 primary and T0.5-T1.5 secondary (Burgasser,

\footnotetext{
${ }^{1}$ Ultracool dwarf is a name normally used for objects with spectral type later than about M7, which could potentially be brown dwarfs, but could also be stars
} 
Sheppard, \& Luhman 2013; Kniazev et al. 2013). Its brightness and proximity are likely to make it an important object for future detailed studies. WISE J088510.83-071442.5 (Luhman 2014) appears to be the coldest brown dwarf known based on its absolute magnitude and colours. These two systems are the closest brown dwarf systems, and the third and fourth closest systems to the Earth (after the $\alpha$ Centauri system and Barnard's star).

\subsection{Brown dwarf spectral sequence}

The study of brown dwarfs has led to a significant extension of the traditional spectral sequence from O-M that was adopted more than 100 years ago (Cannon \& Pickering 1901). Objects such as GD 165B and Gl 229B clearly had quite different spectra and were cooler objects than any M dwarfs. This was recognised by the adoption of the new spectral classes $\mathrm{L}$ and $\mathrm{T}$. The motivation for this and the reasons for the choice of those letters are described by Kirkpatrick et al. (1999). The sequence has been further extended by the recent recognition of even cooler objects that have been assigned to the new spectral class Y (Cushing et al. 2011).

Figure 4 shows the main features of the spectral sequence from M9 to T7.5 with the main absorbing species indicated.

\subsubsection{M dwarfs}

The $\mathrm{M}$ spectral classification has been recognised from the early days of astronomical spectroscopy. While most M dwarfs are stars, young objects of late $M$ spectral types can be brown dwarfs (as shown in Figure 1). The modern classification scheme for M-dwarfs is based on that of Boeshaar (1976) extended by Boeshaar \& Tyson (1985) and Kirkpatrick, Henry \& McCarthy (1991) to spectral type M9.5. The Kirkpatrick et al. (1991) spectral classification is based on the spectral region from $630-900 \mathrm{~nm}$. The spectral standards chosen for late $\mathrm{M}$ types are listed in Table 1.

The $\mathrm{M}$ spectral class is characterised by the presence of bands of $\mathrm{TiO}$ and $\mathrm{VO}$. TiO bands increase in strength up to spectral type M6, and VO becomes strong in the latest types.

In the near infrared (near-IR) $\mathrm{M}$ dwarfs show broad absorptions due to $\mathrm{H}_{2} \mathrm{O}$ centred around 1.4 and $1.9 \mu \mathrm{m}$ increasing in strength with later spectral types. Late $\mathrm{M}$ dwarfs also show Na I and K I absorptions in the 1.15-1.25 $\mu \mathrm{m}$ region. $\mathrm{FeH}$ absorption is present in the Wing-Ford band at $1 \mu \mathrm{m}$ as well as the E-A band in the $1.6 \mu \mathrm{m}$ region (Hargreaves et al. 2010). CO absorption is present at $2.3 \mu \mathrm{m}$.

\subsubsection{L dwarfs}

The $\mathrm{L}$ dwarf class is disinguished by the weakening and disappearance of the $\mathrm{TiO}$ and $\mathrm{VO}$ bands that are distinctive of M dwarfs. TiO has disappeared by L6 and VO by L4. A classification scheme for $L$ dwarfs based on the optical spectral region $(630-1000 \mathrm{~nm})$ is described by Kirkpatrick et al. (1999). It lists spectral standards for classes L0 to L8 (see Table 1) and classification is based on the weakening
$\mathrm{TiO}$ and $\mathrm{VO}$ bands, changes in $\mathrm{CrH}$ and $\mathrm{FeH}$ bands $\mathrm{CrH}$ is strongest at L5) and the alkali metals, with $\mathrm{Cs} \mathrm{I}$ and $\mathrm{Rb}$ I lines increasing in strength to later types.

Spectral classification of $\mathrm{L}$ dwarfs in the near-IR is discussed by Reid et al. (2001), Geballe et al. (2002) and Nakajima, Tsuji, \& Yanagisawa (2004). Kirkpatrick et al. (2010) defined a set of spectral standards for the near-IR for spectral types M0 - L9. The near-IR region shows broad absorption bands of $\mathrm{H}_{2} \mathrm{O}$ increasing in strength towards later spectral types.

While methane in the $1-2.5 \mu \mathrm{m}$ region is not seen until spectral type $\mathrm{T}$, the stronger methane $\nu_{3}$ band in the $3.3 \mu \mathrm{m}$ region is observable in late $\mathrm{L}$ dwarfs (Noll et al. 2000; Schweitzer et al. 2002; Stephens et al. 2009).

The physical basis for the M-L transition is thought to be the formation of condensates. At temperatures just below $2000 \mathrm{~K}$ the condensation of Ti bearing species such as $\mathrm{CaTiO}_{3}$ (perskovite) and $\mathrm{Ti}_{2} \mathrm{O}_{3}$ removes $\mathrm{TiO}$ from the gas phase, and at slightly lower temperatures VO condenses as solid VO (Burrows \& Sharp 1999; Lodders 2002). Species such as enstatite $\left(\mathrm{MgSiO}_{3}\right)$, forsterite $\left(\mathrm{Mg}_{2} \mathrm{SiO}_{4}\right)$, spinel $\left(\mathrm{MgAl}_{2} \mathrm{O}_{4}\right)$ and solid iron also condense and these produce the dust clouds that are necessary to explain the spectra and colours of L dwarfs (Allard et al. 2001; Marley et al. 2002; Tsuji 2002)

\subsubsection{T dwarfs}

The $\mathrm{T}$ dwarf class is characterised by the appearance of methane $\left(\mathrm{CH}_{4}\right)$ absorption features in the near-IR region (1$2.5 \mu \mathrm{m})$. Methane first becomes apparent in early $\mathrm{T}$ dwarfs due to features at 1.67 and $2.2 \mu \mathrm{m}$ which represent the Qbranches of the strongest methane bands $2 v_{3}$ at $1.67 \mu \mathrm{m}$ and $v_{2}+v_{3}$ at $2.2 \mu \mathrm{m}$. This is accompanied by weakening of the $\mathrm{CO}$ absorption at $2.3 \mu \mathrm{m}$.

At later types broad methane absorptions develop due to the complex methane band systems, the octad (8 ground-state bands in the 2.1-2.4 $\mu \mathrm{m}$ region; Hilico et al. 2001) and the tetradecad (14 ground-state bands in the 1.6-2.0 $\mu \mathrm{m}$ region; Nikitin et al. 2013a). These ground-state bands are associated with large numbers of hot bands. Methane absorption is also present at around $1.4 \mu \mathrm{m}$ (the icosad - 20 groundstate bands) and $1.15 \mu \mathrm{m}$ (the triacontad - 30 ground-state bands). Bailey, Ahlsved, \& Meadows (2011) provides a more detailed description of the methane spectrum.

In late $\mathrm{T}$ dwarfs the broad $\mathrm{CH}_{4}$ and $\mathrm{H}_{2} \mathrm{O}$ absorptions deepen and combine to leave a spectrum defined by approximately triangular peaks at $1.08 \mu \mathrm{m}, 1.27 \mu \mathrm{m}$ and $1.58 \mu$ (the 'windows' between the deep absorptions), as well as a weaker peak at about $2.1 \mu \mathrm{m}$. T dwarf spectra are also shaped by the collision-induced absorption due to $\mathrm{H}_{2}-\mathrm{H}_{2}$ pairs (Borysow 2002; Abel et al. 2011) which depresses the $2 \mu \mathrm{m}$ peak, and by the far wings of very strong $\mathrm{Na} \mathrm{I}$ and $\mathrm{K} \mathrm{I}$ lines in the optical (Burrows \& Volobuyev 2003; Allard et al. 2003) which absorb at wavelengths up to $\sim 1 \mu \mathrm{m}$.

Classification schemes for $\mathrm{T}$ dwarfs based on near-IR spectra, were developed by Burgasser et al. (2002) and Geballe 


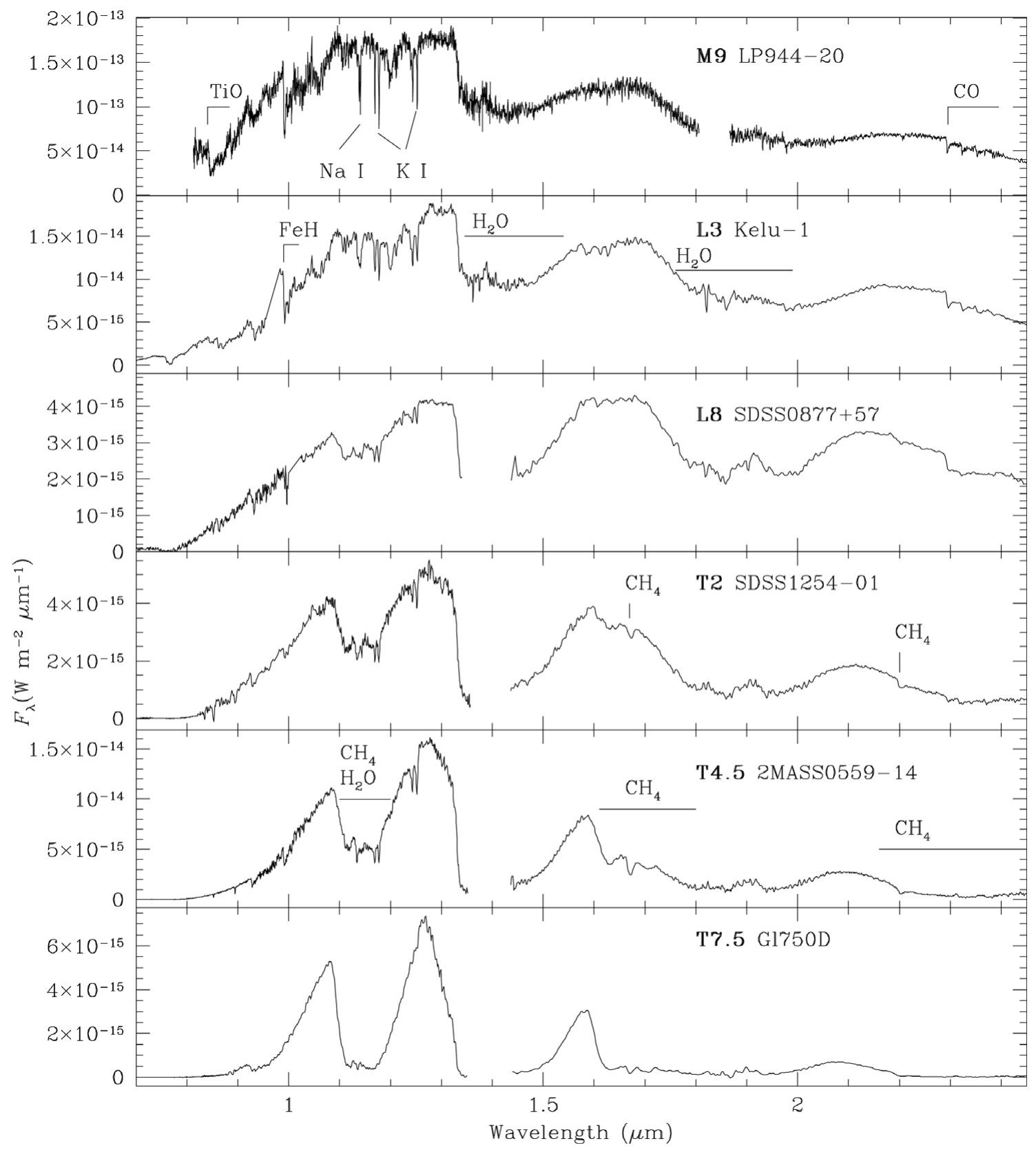

Figure 4. Spectra of ultracool dwarfs from M9 to T7.5. The species responsible for the main absorption features are indicated. Spectral data is from Burgasser et al. (2003), Cushing, Rayner, \& Vacca (2005), Geballe et al. (2001), Geballe et al. (2002), Leggett et al. (2000), Leggett et al. (2001), Leggett et al. (2002), Rayner, Cushing, \& Vacca (2009), Ruiz, Leggett, \& Allard (1997)

et al. (2002) and the two schemes were unified in Burgasser et al. (2006a). That work gives a set of spectral standards for T0-T8 (see Table 1). The main features used for classification are the increasing depths of the $\mathrm{H}_{2} \mathrm{O}$ and $\mathrm{CH}_{4}$ bands towards later classes. A parallel optical classification scheme based on the 630-1010 $\mathrm{nm}$ region is described by Burgasser et al. (2003) and is based on some of the same spectral standards used in the near-IR.

The transition from $\mathrm{L}$ to $\mathrm{T}$ is associated with the switch in chemical equlibirum between $\mathrm{CO}$ and $\mathrm{CH}_{4}$ (Lodders 2002; Burrows \& Sharp 1999) that occurs at about $1400 \mathrm{~K}$ at 1 bar pressure, with $\mathrm{CO}$ being more stable above this temperature, and $\mathrm{CH}_{4}$ being favoured at lower temperatures. However, the transition is also associated with a clearing of the dust clouds that are important in L dwarfs (Allard et al. 2001; Burgasser et al. 2002).

\subsubsection{Y dwarfs}

The possible existence of objects even cooler than the $\mathrm{T}$ dwarfs was investigated in models by Burrows, Sudarsky, $\&$ Lunine (2003). Among the features suggested as marking the transition to a new spectral class, were the appearance 
Table 1. Mean properties and spectral standards for late M to Y dwarfs

\begin{tabular}{|c|c|c|c|c|c|c|}
\hline & $\mathrm{T}_{\text {eff }}$ & $\mathrm{M}_{J}$ & $\mathrm{M}_{H}$ & $\mathrm{M}_{K}$ & Spectral standard & Refs \\
\hline M7 & 2633 & 10.31 & 9.94 & 9.50 & 2MASS J16 553 529-0 823401 (Gl 644C, vB8) & 1,7 \\
\hline M8 & 2520 & 10.99 & 10.40 & 9.88 & 2MASS J19 $165762+0509021$ (G1 752B, vB10) & 1,7 \\
\hline M9 & 2465 & 11.80 & 11.15 & 10.62 & 2MASS J14 284 323+3 310391 (LHS 2924) & 1,7 \\
\hline L0 & 2438 & 11.69 & 11.04 & 10.46 & 2MASS J03 $454316+2540233$ & 2,7 \\
\hline $\mathrm{L} 1$ & 2329 & 11.87 & 11.26 & 10.66 & 2MASS J07 464 256+2 000321 & 2,7 \\
\hline $\mathrm{L} 2$ & & 12.18 & 11.45 & 10.82 & 2MASS J13 054 019-2 541059 (Kelu-1) & 2,7 \\
\hline L3 & 1948 & 12.81 & 11.97 & 11.26 & 2MASS J1 $1463449+2230527$ & 2,7 \\
\hline L4 & 1801 & 12.83 & 12.14 & 11.25 & 2MASS J11 $550087+2307058$ & 2,7 \\
\hline L5 & 1686 & 13.44 & 12.61 & 11.96 & 2MASS J12 $281523-1547342$ & 2,7 \\
\hline L6 & 1501 & 14.12 & 13.05 & 12.00 & 2MASS J08 $503593+1057156$ & 2,7 \\
\hline L7 & 1446 & 14.67 & 13.70 & 12.89 & 2MASS J02 $059240-1159296$ & 2,7 \\
\hline L8 & 1445 & 14.68 & 13.77 & 13.05 & 2MASS J16 322 911-1904 407 & 2,7 \\
\hline L9 & & 14.33 & 13.48 & 12.73 & 2MASS J02 $550327-4700509$ & 3,7 \\
\hline T0 & 1370 & 14.24 & 13.52 & 13.17 & SDSS J120 747.17+024 424.8 & 4,7 \\
\hline $\mathrm{T} 1$ & & 14.37 & 13.81 & 13.62 & SDSS J083 717.21-000 018.0 & 4,7 \\
\hline $\mathrm{T} 2$ & 1328 & 14.43 & 13.88 & 13.58 & SDSS J125 453.90-012 247.4 & 4,7 \\
\hline $\mathrm{T} 3$ & & & & & 2MASS J12 095 613-1004008 & 4,7 \\
\hline $\mathrm{T} 4$ & 1251 & 15.04 & 14.41 & 14.13 & 2MASS J22 $541892+3123498$ & 4,7 \\
\hline T5 & 1185 & 14.43 & 14.66 & 14.81 & 2MASS J15 $031961+2525196$ & 4,7 \\
\hline T6 & 1001 & 15.22 & 15.56 & 15.77 & SDSS J1642 414.37+002 915.6 & 4,7 \\
\hline $\mathrm{T} 7$ & 820 & 15.54 & 15.97 & 16.01 & 2MASS J07 $271824+1710012$ & 4,7 \\
\hline $\mathrm{T} 8$ & 638 & 16.43 & 16.82 & 16.93 & 2MASS J04 $151954-0935066$ & 4,8 \\
\hline T9 & 565 & 18.39 & 18.77 & 18.89 & UGPS J072 227.51-054 031.2 & 5,8 \\
\hline Y0 & 371 & 20.09 & 20.60 & 20.70 & WISE J173 835.52+273 258.9 & 5,8 \\
\hline $\mathrm{Y} 1$ & & & & & WISE J035 000.32-565 830.2 & 6 \\
\hline
\end{tabular}

References. First reference is to adoption of the spectral standard, and the second reference is to the source of the mean absolute magitudes.

1. Kirkpatrick et al. (1991), 2. Kirkpatrick et al. (1999), 3. Kirkpatrick et al. (2010) 4. Burgasser et al. (2006a), 5. Cushing et al. (2011), 6. Kirkpatrick et al. (2012), 7. Dupuy \& Liu (2012), 8. Dupuy \& Kraus (2013)

Notes.

Mean effective temperatures are from the data of Figure 6. Spectral standards are those adopted for optical classification up to spectral class L8, and for near-IR classification for L9 and later. Near-IR spectral standards for earlier types can be found in Kirkpatrick et al. (2010). Spectral data is available for download for most of these objects (and other late-type dwarfs) at:

SpeX Prism Spectral Libraries (A. Burgasser)

— http://pono.ucsd.edu/ adam/browndwarfs/spexprism/

IRTF Spectral library (M.R. Cushing)

— http://irtfweb.ifa.hawaii.edu/ $\sim$ spex/IRTF_Spectral_Library/

$\mathrm{L}$ and $\mathrm{T}$ dwarf data archive (S.K. Leggett)

— http://staff.gemini.edu/ sleggett/LTdata.html

NIRSPEC Brown Dwarf Spectroscopic Survey (I.S. McLean)

— http://www.astro.ucla.edu/ mclean/BDSSarchive

Keck LRIS specra of late-M, L and T dwarfs (I.N. Reid)

— http://www.stsci.edu/ inr/ultracool.html

of $\mathrm{NH}_{3}$ absorption, the condensation of $\mathrm{H}_{2} \mathrm{O}$ clouds, and the development of redder near-IR colours reversing the trend in T dwarfs. A number of of very cool dwarfs were found in the CFBDS and UKIDSS surveys (Warren et al. 2007; Delorme et al. 2008b; Burningham et al. 2008). Lucas et al. (2010) reported the discovery of an even cooler object UGPS 0722-05 which they suggested should be classified as T10, and could in the future be regarded as the first example of a new spectral type.

In 2011, Cushing et al. (2011) reported the 'Discovery of Y-dwarfs'. Several objects identified using the WISE satellite were found to be of later spectral types than UGPS 0722-05. They reclassified UGPS $0722-05$ as the T9 spectral standard, and classified six new objects as Y dwarfs with WISE $1738+27$ as the Y0 standard. Kirkpatrick et al. (2012) report several more $\mathrm{Y}$ dwarfs and added a spectral standard for the Y1 class (see Table 1). Other reported Y dwarfs are WISE J1639-68 (Tinney et al. 2012) and the white dwarf companion WD 0806-661 B (Luhman, Burgasser, \& Bochanski 2011; Luhman et al. 2012). The high proper motion object WISE J085 510.83-071 442.5 (Luhman 2014) has absolute magnitude and colours suggesting it is the coolest known Y dwarf with an effective temperature of 225 $260 \mathrm{~K}$.

All Y dwarfs are very faint objects ( $\mathrm{J}$ mag of 19 or fainter) and so the quality of available spectra are limited. They resemble the late T dwarfs, but the 'window' features (particularly that at $1.27 \mu \mathrm{m}$ ) become increasingly narrow with later spectral types. The $\mathrm{NH}_{3}$ absorptions expected at $\sim 1.53$ and $\sim 1.03 \mu \mathrm{m}$ are not seen at 
the levels predicted by equilibrium chemistry (Leggett et al. 2013).

\subsection{Photometry}

Photometric data on ultracool dwarfs in the near-IR is available for a large number of objects. The database of $\mathrm{L}, \mathrm{T}$ and Y dwarfs at DwarfArchives.org, for example, lists 1281 objects most of which have JHK magnitudes. When interpreting photometric data at JHK it is important to note that there are several different JHK systems in use. In particular the 2MASS (Carpenter 2001) and MKO (Simons \& Tokunaga 2002) systems are both widely used in brown dwarf research. The 2MASS system uses a significantly shorter wavelength and narrower $\mathrm{K}_{s}$ band compared to the $\mathrm{K}$ band of the MKO system. Transformations between the systems derived from data on stars (Carpenter 2001) are unlikely to be valid for the unusual energy distributions seen particularly in the $T$ dwarfs. Stephens \& Leggett (2004) provide a set of transformations between photometric systems specifically for $\mathrm{L}$ and $\mathrm{T}$ dwarfs that can be used if the spectral type is known.

Much of the energy in ultracool dwarfs is in the mid infrared, and photometry for these wavelengths has become increasingly available from Spitzer/IRAC (Patten et al. 2006; Leggett et al. 2007, 2010) and the WISE all sky catalog (Wright et al. 2010).

These objects are relatively nearby and so parallax measurements of good quality are generally feasible allowing absolute magnitudes to be derived. Conventional CCD parallax methods can be used for the earlier type objects (e.g. Dahn et al. 2002; Vrba et al. 2004; Andrei et al. 2011). Infrared parallaxes can be measured for the latest type objects (Tinney, Burgasser, \& Kirkpatrick 2003; Dupuy \& Liu 2012; Marsh et al. 2013). The recent compilation by Dupuy \& Liu (2012) includes absolute magnitudes in the near and mid infrared for 314 objects with known parallaxes. Mean absolute magnitudes from this compilation in the MKO JHK systems are given in Table 1 supplemented by those of Dupuy \& Kraus (2013) for the latest spectral types.

Figure 5 shows the $\mathrm{J}-\mathrm{K}$ against $\mathrm{M}_{K}$ colour magnitude diagram for $\mathrm{M}$ to $\mathrm{Y}$ dwarfs. A distinctive feature of the diagram is the behaviour at the L/T transition. Generally the $\mathrm{J}-\mathrm{K}$ colour becomes slowly redder with later spectral types through $\mathrm{M}$ and $\mathrm{L}$, but then rapidly turn bluer through the early $\mathrm{T}$ spectral types. The limited photometry available for $\mathrm{Y}$ dwarfs suggests a turn back to redder colours.

In the $\mathrm{J}$ band a significant brightening with later spectral type can be seen (Dahn et al. 2002; Tinney, Burgasser, \& Kirkpatrick 2003). In the mean data of Table 1 it can be seen that types L9 to T2 are all brighter at J than L6 and L7. Tsuji \& Nakajima (2003) suggested that this may be an artifact of observing objects with different masses and ages, and not a feature seen in a single cooling track. Studies of binary brown dwarfs whose components straddle the L/T boundary, however, show 'flux reversals' where the cooler component is brighter in the $1-1.3 \mu \mathrm{m}$ region (Burgasser et al. 2006b;

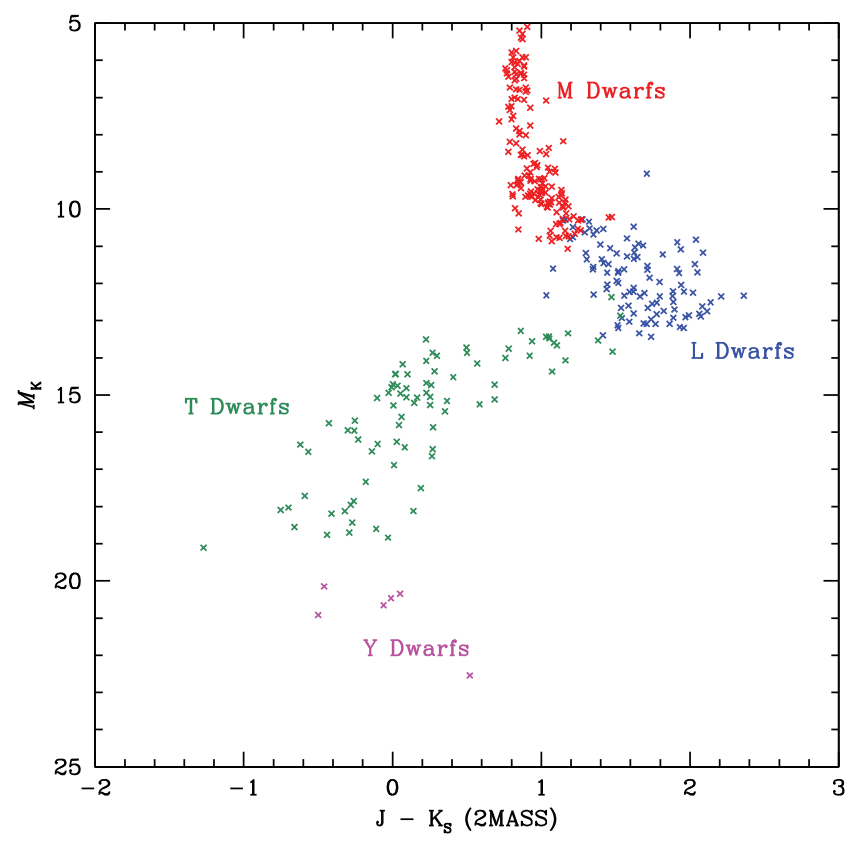

Figure 5. Colour magnitude diagram $\left(\mathrm{J}-\mathrm{K}\right.$ against $\mathrm{M}_{K}$ ) for late type dwarfs. Most of the data is taken from Dupuy \& Liu (2012). Data on late T and Y dwarfs is from Dupuy \& Kraus (2013) and has been roughly converted to the 2MASS system according to Stephens \& Leggett (2004). Additional data on earlier type $\mathrm{M}$ dwarfs has been added from the compilation of Reid (http://www.stsci.edu/ $1 \mathrm{nr} / \mathrm{cmd} . h t m l)$ based on photometry from Leggett (1992) and converted to the 2MASS system using relations in Carpenter (2001).

Liu et al. 2006; Looper et al. 2008) showing that the effect is a real intrinsic features of the $\mathrm{L} / \mathrm{T}$ transition.

\subsection{Effective temperatures}

The effective temperature of ultracool dwarfs can be determined by two main methods. The first way is to use photometry and parallax measurements to determine the bolometric luminosity. A temperature can then be derived if the radius is known. We don't have direct radius measurements for most of these objects, but as shown in Figure 2, models predict that the radius of brown dwarfs varies little with mass and age, so model based radius constraints can be used to determine effective temperature.

The other way to determine effective temperatures is to fit observed spectra to those predicted by model atmospheres. This is likely to be most reliable if the observations cover a large wavelength range that includes a substantial fraction of the luminosity, and for brown dwarfs this means including the mid-IR as well as the near-IR (e.g. Stephens et al. 2009).

Figure 6 is a compilation of effective temperature measurements from the literature using both of these methods. It shows reasonable agreement betwee the various determinations. A feature of this diagram is that, while the general trend is decreasing temperature with later spectral type, the 


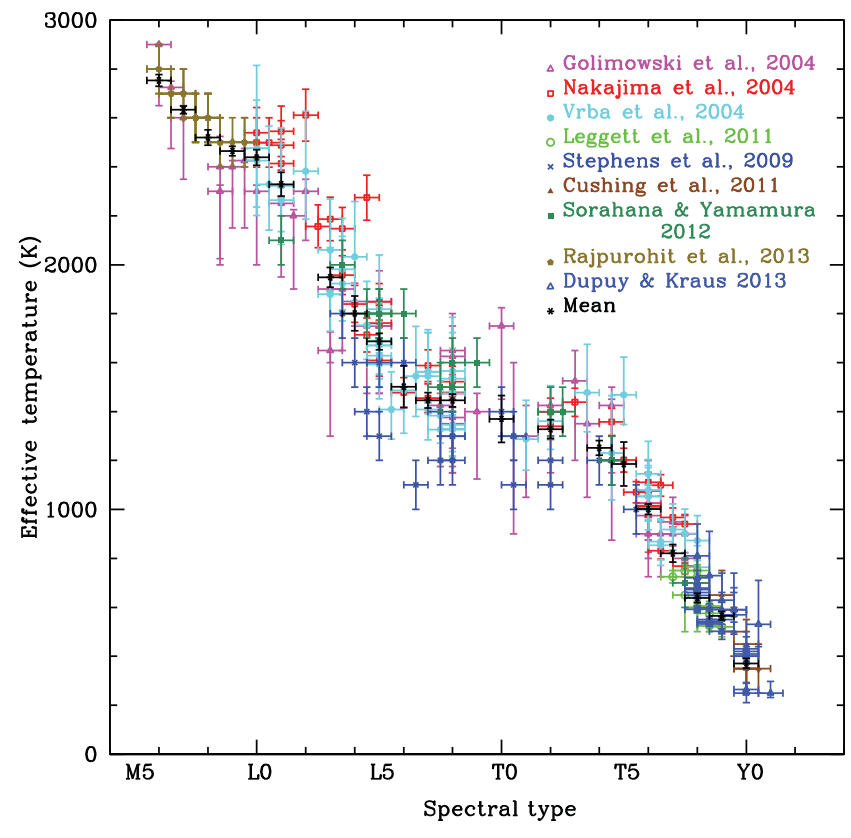

Figure 6. Effective temperatures plotted against spectral type. The effective temperatures are determined from bolometric luminosities (Vrba et al. 2004; Golimowski et al. 2004; Nakajima, Tsuji, \& Yanagisawa 2004; Dupuy \& Kraus 2013) or from fitting models to observed spectra (Stephens et al. 2009; Leggett et al. 2011; Cushing et al. 2011; Sorahana \& Yamamura 2012; Rajpurohit et al. 2013). Optical spectral types are used up to L8, and infrared spectral types for L9 and later. Late T and Y dwarf spectral types are from Kirkpatrick et al. (2012). Spectral types are shown with error bars of \pm 0.5 subtypes. Mean values are given for spectral types that had more than 3 measurements. Where no error estimate was given in the original publication an error bar of $\pm 100 \mathrm{~K}$ has been shown.

temperature actually changes little over the $\mathrm{L} / \mathrm{T}$ transition from about L6 to T4. This suggests that the spectral changes seen over this range are due to the clearing of dust rather than to the direct effect of changing temperatures.

The mean effective temperatures for each spectral type from the data of Figure 6 have been included in Table 1.

The L/T transition shows up particularly clearly when the near-IR colours and spectral types are plotted against effective temperature using the mean values given in Table 1 . The $\mathrm{J}-\mathrm{K}$ and $\mathrm{J}-\mathrm{H}$ colours and the spectral type all vary dramatically over the effective temperature range from 1200 $1500 \mathrm{~K}$, and show much less variability at other temperatures as shown in Figure 7. The changes are thought to be mostly due to the disappearance of dust clouds as the atmospheres cool, but it is not clear why this should appear as such a sharp transition. Cloud models (to be described in Section $4.4-$ the red and blue lines) show much more gradual changes than those observed.

\subsection{Variability}

Variability has been reported in a number of L dwarfs (Clarke, Tinney, \& Covey 2002; Koen 2006; Lane et al. 2007; Heinze et al. 2013). The amplitudes are typically $\sim 1 \%$ and the vari-

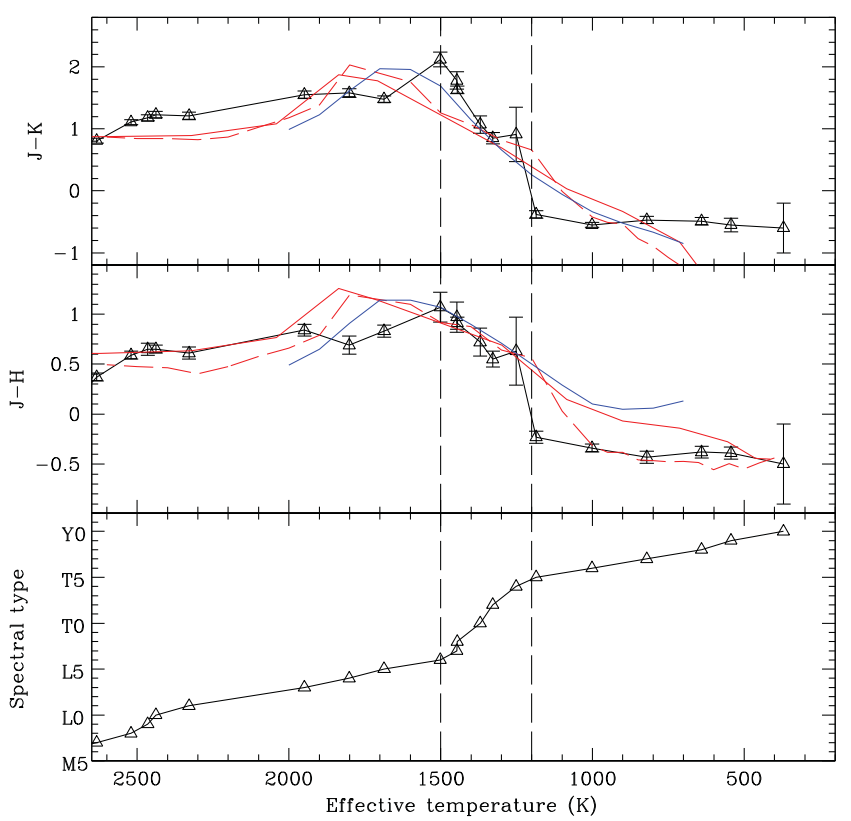

Figure 7. The $\mathrm{L} / \mathrm{T}$ Transition. Over the small range of effective temperature from 1200-1500 K the observed colours and spectral types of ultracool dwarfs (black) vary through a large range. Models (red and blue lines, see section 4.4) show much more gradual changes. The data for this plot are that of Table 1. Model results are from the BT-Settl models (Allard et al. 2007, 2012) for $\log g=5$ (solid red line) and for a 3 Gyr isochrone (red dashed line), and the Unified Cloudy Model (Tsuji 2002, 2005) for $\log g=5$ and $\mathrm{T}_{c r}=1800 \mathrm{~K}$ (blue line).

ations are quasi-periodic with periods of a few hours. The variability is generally attributed to rotational modulation either due to patchy clouds, or magnetic spots.

Two early T dwarfs have been observed to show larger amplitude variability (Artigau et al. 2009; Radigan et al. 2012). In the case of the T2.5 dwarf 2MASS 2139+02 an amplitude of up to $26 \%$ was observed with a period of 7.72 hours. The large amplitude in these early $\mathrm{T}$ objects is suggested to be indicative of patchy cloud regions arising during the clearing of clouds associated with the L/T transition as suggested by Marley, Saumon, \& Goldblatt (2010). A further example reported recently (Gillon et al. 2013) is variability in the cooler component of the 2 pc binary brown dwarf WISE J1049-53 (Luhman 16).

Buenzli et al. (2012) have reported Spitzer and HST observations of variability with a $1.4 \mathrm{hr}$ period in the T6.5 dwarf 2MASS 2228-43, confirming a ground-based detection of this period by Clarke et al. (2008). They find phase shifts between variations at different wavelengths which can provide a probe of the vertical atmospheric structure.

Recently Crossfield et al. (2014) have used time resolved near-infrared spectroscopy around the rotation period to derive a global 2D map of the brighteness distribution of Luhman 16B using Doppler imaging techniques. The map reveals structure that may be due to patchy clouds. 


\section{EXOPLANETS}

\subsection{History and properties}

Since the discovery of the first planets orbiting normal stars (Mayor \& Queloz 1995; Marcy et al. 1997) the rate of discovery has steadily increased to more than 1800 confirmed planets according to The Extrasolar Planets Encyclopedia (exoplanet.eu - Schneider et al 2011) as at July 2014. In addition more than 3000 planet candidates have now been found by the Kepler mission (Batalha et al. 2013). The latter are not yet confirmed planets, but it is estimated that the false positive rate for Kepler planet candidates is likely to be $\sim 10 \%$ (Morton \& Johnson 2011; Fressin et al. 2013).

While there are a large number of planets, observations of their atmospheres are much more difficult than for the brown dwarfs just considered. The vast majority of planet detections and observations are by indirect methods, such as radial velocity measurements of the host star, and transit measurements. These provide information on the orbit, mass and radius (for transitting planets). However, apart from a small number of directly imaged planets, we don't yet have the capability to resolve planets from their stars in order to measure their spectra. At present most of our data on the spectra of exoplanets comes from analysis of unresolved planets that require extracting signals that are a small fraction of that from the host star.

A recent review of exoplanet detection methods is given by Wright \& Gaudi (2013). All methods currently used are subject to biases. The radial velocity (RV) technique that has been used for the majority of exoplanet discoveries favours the detection of massive planets and short period orbits. The majority of RV detected planets are therefore giant planets, but at short periods this method can detect planets down to a few Earth masses. When corrections are made for incompleteness the statistics show that planet frequency increases for decreasing mass (Howard et al. 2010; Wittenmyer et al. 2011). This is consistent with the increasing planet frequency at small sizes shown by analysis of the Kepler planet candidates (Howard et al. 2012). Ground-based transit searches (e.g. Bakos et al. 2004; Pollacco et al. 2006) are strongly biased toward finding large short period planets (i.e. hot Jupiters).

Our current ability to characterise exoplanet atmospheres is largely limited limited to giant planets and to planets with high temperatures $(\mathrm{T}>\sim 1000 \mathrm{~K})$. In most cases these are hot Jupiters, i.e. massive planets that are hot because they are close to their star, or are directly imaged massive planets that are hot because they are young planets still cooling. There are a few cases of lower mass planets, for example Neptune/Uranus mass planets such as GJ 3470b and GJ 436b and two examples of transiting super-Earths, GJ 1214b (Charbonneau et al. 2009) and HD $97658 \mathrm{~b}$ for which characterisation observations have been made.

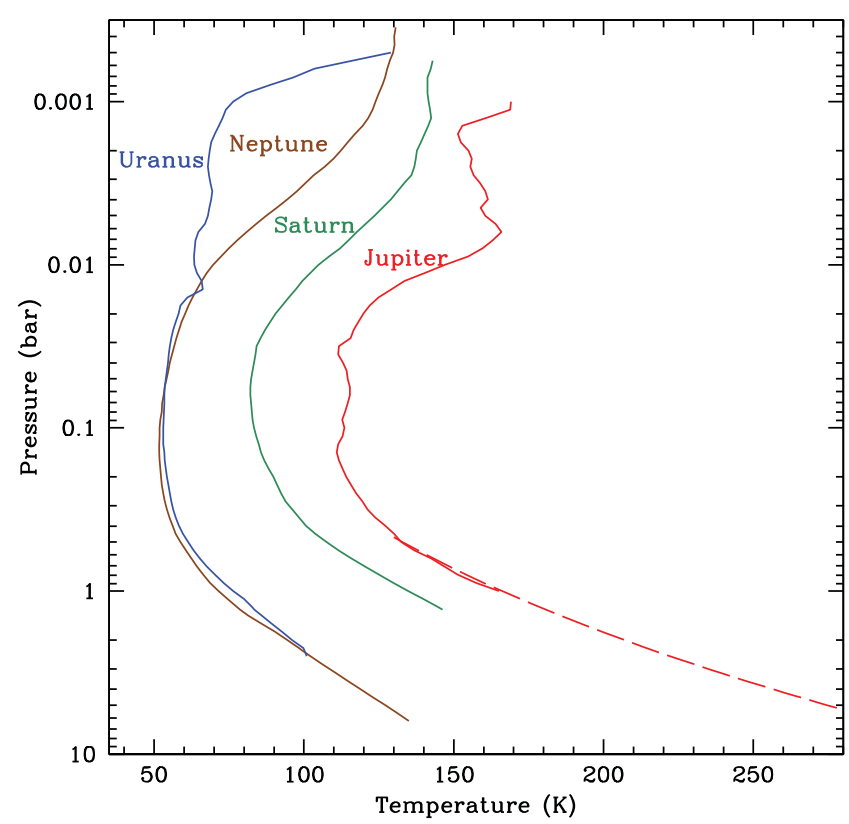

Figure 8. Temperature profiles of the Solar system giant planet atmospheres from Voyager radio occultation measurements (Lindal 1992), and from the Galileo probe for Jupiter (dashed line - Seiff et al. 1998).

\subsection{Solar system giant planets}

We do, however, know of several giant planets that have been studied in considerable detail, the giant planets in our own Solar system. It is useful to briefly review their properties. All the giant planets have atmospheres composed of hydrogen and helium and are enriched in heavy elements with respect to the solar composition. In the case of Jupiter measurements with the Galileo probe show $\mathrm{C}, \mathrm{N}, \mathrm{S}, \mathrm{Ar}, \mathrm{Kr}$, and Xe enriched by factors of 2 to 4 relative to solar abundances (Owen et al. 1999; Wong et al. 2004). Carbon is enriched relative to its solar value by 7 times in Saturn (Flasar et al. 2005) and by $30-40$ times in Uranus and Neptune (Lodders \& Fegley 1994).

All the Solar system giant planet atmospheres have directly measured temperature structures from radio occultation measurements (Lindal 1992), and from the Galileo probe (Seiff et al. 1998) in the case of Jupiter (see Figure 8). All the planets have clouds with the main cloud deck at about 0.75 bar in Jupiter (Banfield et al. 1998; Kedziora-Chudczer \& Bailey 2011), 2.5 bar in Saturn (Fletcher et al. 2011) and $\sim 2$ bar in Uranus and Neptune (Irwin, Teanby, \& Davis 2010).

Near-IR spectra of the giant planets are shown in Figure 9. All of these are dominated by absorption band systems due to methane $\left(\mathrm{CH}_{4}\right)$ centred around 1.15, 1.4, 1.7 and $2.3 \mu \mathrm{m}$, and are bright in the window regions between these absorptions. In this respect the spectra resemble those of late $T$ dwarfs, and the T9 dwarf UGPS 0722-05 is shown in Figure 9 for comparison. Jupiter also show absorption due to $\mathrm{NH}_{3}$ at around $1.55 \mu \mathrm{m}$. All the planets also show collision induced absorption due to $\mathrm{H}_{2}-\mathrm{H}_{2}$ pairs, which at these low temperatures shows up as a distinctive broad feature at around $2.12 \mu \mathrm{m}$. 


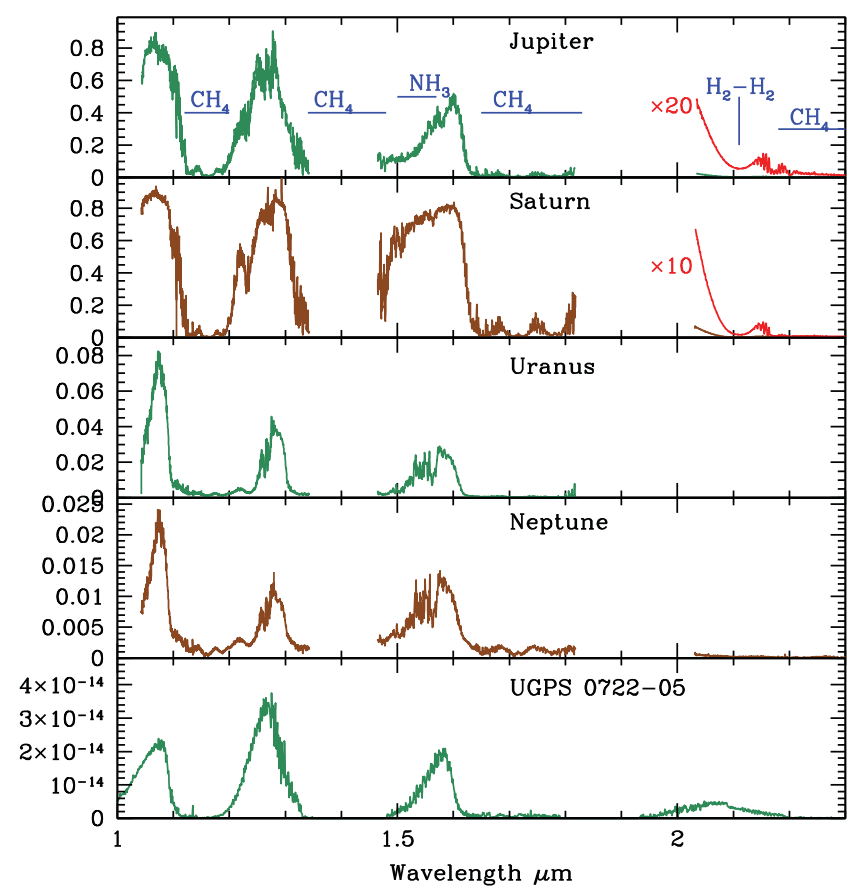

Figure 9. Near-IR reflected light spectra of the Solar system giant planets Jupiter, Saturn, Uranus and Neptune (plotted as radiance factor I/F). The data are from IRIS2 on the Anglo-Australian Telescope as described by Kedziora-Chudczer \& Bailey (2011). The red curves show the weak K-band spectra of Jupiter and Saturn scaled up by factors of 10 and 20. The spectrum of the T9 dwarf UGPS 0722-05 is shown for comparison using data from Bochanski et al. (2011).

This depresses the brightness in the methane window that would otherwise be present at around $2 \mu \mathrm{m}$, and makes all the planets quite faint in the K-band compared with the $\mathrm{J}$ and $\mathrm{H}$ bands.

Other species present in the atmospheres at trace levels and detected in longer wavelength spectra include $\mathrm{PH}_{3}$ and $\mathrm{AsH}_{3}$ in Jupiter and Saturn (Fletcher et al. 2011), and hydrocarbons such as $\mathrm{C}_{2} \mathrm{H}_{2}$ and $\mathrm{C}_{2} \mathrm{H}_{6}$ in the stratospheres (Hesman et al. 2009; Greathouse et al. 2011).

\subsection{Observing exoplanet atmospheres}

\subsubsection{Direct spectroscopy of resolved planets}

A number of 'planets' have been discovered through direct imaging of young objects using ground-based adaptive optics or the Hubble Space Telescope. These include the companion of the brown dwarf 2MASSW J1207 334-393 254 (usually referred to as $2 \mathrm{M} 1207 \mathrm{~b}$ - Chauvin et al. 2005), and the four planets of HR 8799 (Marois et al. 2008, 2010).

The classification of some of these objects as planets is controversial. Although 2M 1207b was announced as the first directly imaged extrasolar planet by its discoverers, it can be argued that it is not a planet because it orbits a brown dwarf, not a star, or because it is unlikely that it formed through the normally understood planet formation process from a disk

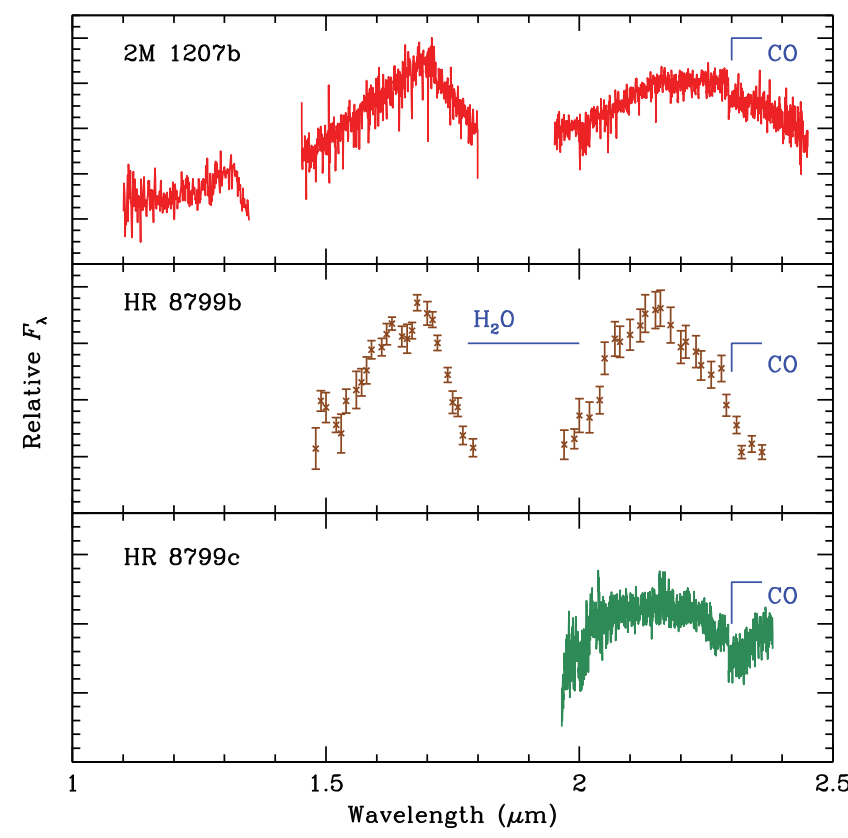

Figure 10. Spectra of the direct imaged planets (or planetary mass objects) 2M 1207b (Patience et al. 2010), HR 8799b (Barman et al. 2011a) and HR $8799 \mathrm{c}$ (Konopacky et al. 2013). The CO bandhead at $2.3 \mu \mathrm{m}$ is apparent in all three objects as well as $\mathrm{H}_{2} \mathrm{O}$ absorption at $\sim 1.9$ and $\sim 1.4 \mu \mathrm{m}$.

around its primary object (Soter 2006). 2M 1207b is usually referred to as a 'planetary mass object' in recent literature.

The classification of such objects as planets also depends on the masses determined by application of evolutionary models, and this critically depends on the age. Marois et al. (2010) use age ranges from $20-160 \mathrm{Myr}$ for HR8799 to derive masses for the planets in the range $5-13 \mathrm{M}_{J u p}$ placing them most likely below the deuterium burning limit. However, an age as high as $\sim 1$ Gyr is suggested by asteroseismology methods (Moya et al. 2010) which would make the objects brown dwarfs rather than planets. A number of recent studies based on dynamics (Moro-Martín, Rieke, \& Su 2010; Sudol \& Haghighipour 2012) and a direct radius determination for HR 8799 (Baines et al. 2012) favour a young age and planetary masses for the companions.

Near-IR spectra have been obtained for 2M 1207b (Mohanty et al. 2007; Patience et al. 2010), the HR 8799 planets (Bowler et al. 2010; Barman et al. 2011a; Oppenheimer et al. 2013; Konopacky et al. 2013) and $\beta$ Pic b (Chilcote et al. 2014). Spectra of $2 M 1207 \mathrm{~b}$ and HR $8799 \mathrm{~b}$ and $\mathrm{c}$ are shown in Figure 10. The spectra show the CO bandhead at $2.3 \mu \mathrm{m}$, and $\mathrm{H}_{2} \mathrm{O}$ absorption at 1.4 and $1.9 \mu \mathrm{m}$ (deepest in HR 8799b). $\mathrm{CH}_{4}$ absorption is either absent or possibly weakly present in HR 8799b. The spectral features are similar to those of mid to late $\mathrm{L}$ dwarfs, which would imply objects of $\mathrm{T}_{\text {eff }}$ $\sim 1400-1600 \mathrm{~K}$.

However, photometry of $2 \mathrm{M} 1207 \mathrm{~b}$ shows it to be very red in $\mathrm{J}-\mathrm{K}$ and underluminous compared with $\mathrm{L}$ dwarfs (Figure 11). This led Mohanty et al. (2007) to suggest that grey extinction by an edge-on disk may be the cause of the 


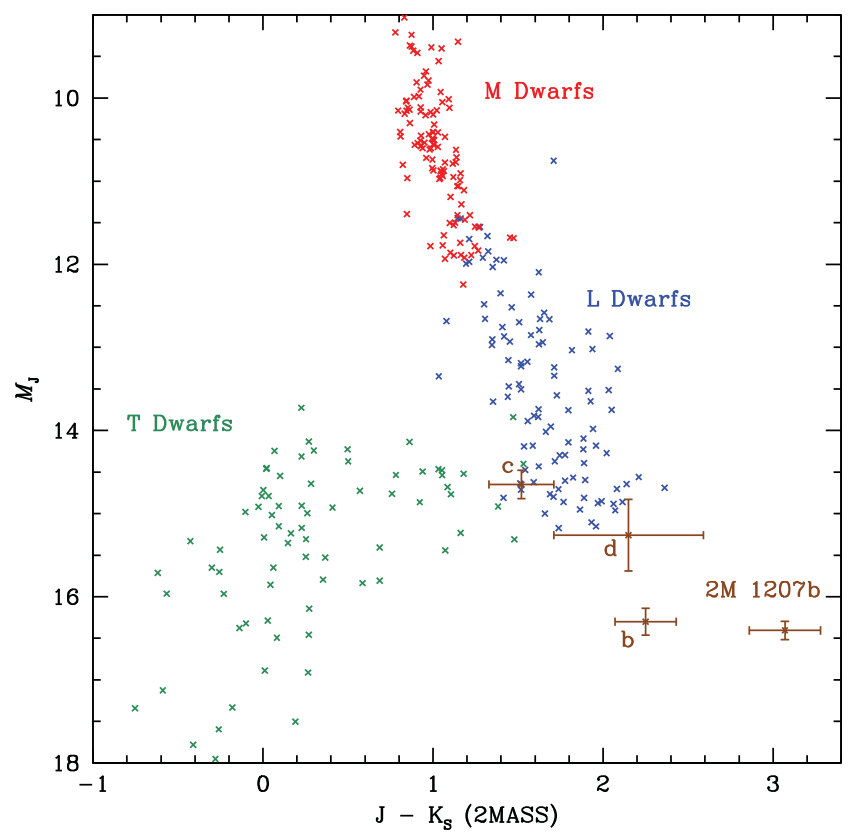

Figure 11. Colour magnitude diagram for $2 \mathrm{M} 1207 \mathrm{~b}$ and the b, c and d planets of HR 8799 (photometry from Chauvin et al. 2005; Marois et al. 2008; Mohanty et al. 2007) compared with field M, L and T dwarfs (from the same data sources as Figure 5).

underluminosity. Photometry of HR $8799 \mathrm{~b}$ show that it is similarly underluminous. Barman et al. (2011b) have shown that it is possible to model the spectrum of $2 \mathrm{M} 1207 \mathrm{~b}$ with a cool $\left(\mathrm{T}_{\text {eff }} \sim 1000 \mathrm{~K}\right)$ model by including clouds and a departure from chemical equilibrium due to vertical mixing that inhibits the formation of methane. Similar models have been fitted to the spectra of HR 8799b (Barman et al. 2011a) and c (Konopacky et al. 2013).

Spectroscopy of $\beta$ Pic $\mathrm{b}$ in the $\mathrm{H}$ band (Chilcote et al. 2014) taken with the Gemini Planet Imager shows spectral structure indicating $\mathrm{H}_{2} \mathrm{O}$ absorption and atmospheric model fits give $\mathrm{T}_{\text {eff }}=1650 \pm 50 \mathrm{~K}$ and $\log g=4.0 \pm 0.25$.

A detection of methane (Janson et al. 2013) has been reported in the planetary mass companion GJ 504b (Kuzuhara 2013). This was achieved using Spectral Differential Imaging with the HiCAIO adaptive optics camera on the Subaru telescope. The companion was found to be much fainter in the $\mathrm{CH}_{4}$ absorption band at $\sim 1.7 \mu \mathrm{m}$ than in other bands indicating a deep methane absorption comparable to that in late $\mathrm{T}$ dwarfs.

\subsubsection{High resolution cross correlation techniques}

Spectral features due to an unresolved extrasolar planet can be detected using high-resolution spectroscopy, and cross correlation techniques to pick out the faint signal due to the planet from the much brighter contribution of the host star. The technique was first used to attempt to detect the reflected light signal in high-resolution optical spectra of hot Jupiters. A possible detection of a planetary signal in $\tau$ Boo was reported (Collier Cameron et al. 1999) but was

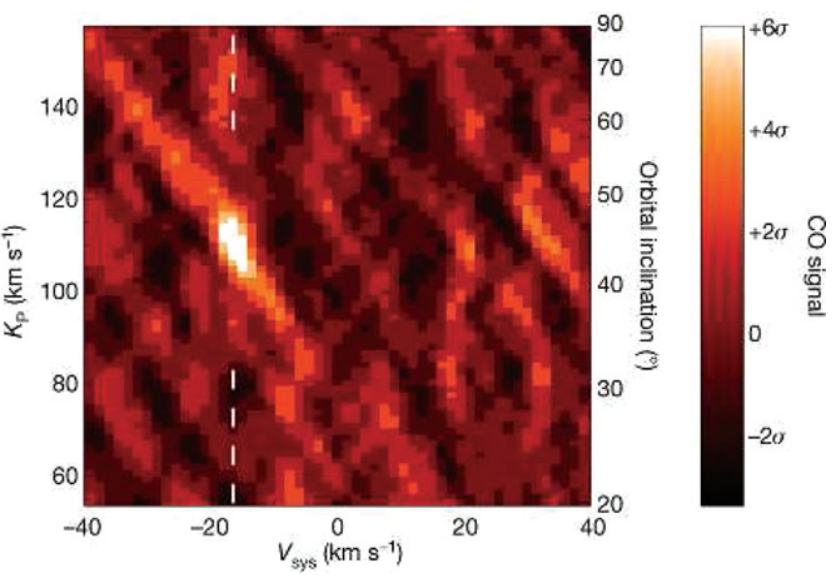

Figure 12. Carbon monoxide cross correlation signal for $\tau$ Boo $b$ (Brogi et al. 2012) as a function of systemic velocity $\left(V_{s y s}\right)$ and radial velocity amplitude of the planet $\left(K_{P}\right)$. A $6.2 \sigma$ signal is seen at $K_{P}=110 \pm 3.2 \mathrm{~km}$ $\mathrm{s}^{-1}$ corresponding to an inclination $i=44.5^{\circ} \pm 1.5$ and a planet mass $M_{P}=$ $5.95 \pm 0.28 M_{\text {Jup }}$ - Reprinted by permission from Macmillan Publishers Ltd: Nature, 486, 502-504, (C) (2012).

not confirmed (Charbonneau et al. 1999; Leigh et al. 2003a) and is inconsistent with the subsequent infrared detections by Brogi et al. (2012) and Rodler, Lopez-Morales, \& Ribas (2012). These studies set upper limits on the geometric albedo of $\tau$ Boo b of 0.3 at $480 \mathrm{~nm}$ (Charbonneau et al. 1999) and 0.39 over $400-650 \mathrm{~nm}$ (Leigh et al. 2003a). Other reflected light studies for a number of the brighter hot Jupiter systems (Collier Cameron et al. 2002; Leigh et al. 2003b; Rodler, Kurster, \& Henning 2008, 2010; Langford et al. 2011) result in similar upper limits on geometric albedo.

Much more successful have been similar studies in the near-IR where it is possible to search for specific molecular absorption features either in the transmission spectrum during transit (Snellen et al. 2010a) or in the thermal emission from the planet (which does not require a transiting planet). In these studies the telluric and stellar absorption features are removed as best as possible and the remaining signal is cross correlated with a template spectrum. The large radial velocity amplitude of the planet causes the absorption features to shift with orbital phase, so that a cross correlation peak can be searched for as a function of radial velocity amplitude $\left(\mathrm{K}_{P}\right)$ and systemic velocity $\left(V_{\text {sys }}\right)$ as shown in Figure 12 (Brogi et al. 2012).

The method determines $\mathrm{K}_{P}$ and thus provides a direct measurement of the planet's mass and the orbital inclination, removing the $\sin i$ uncertainty for non-transiting planets. If the planet is transiting the results can be checked against those determined from transit analysis. The systemic velocity is also determined and should agree with that measured for the host star. Table 2 list the detections reported. For two objects ( $\tau$ Boo b and HD 189733b) there are independent results from two studies that are in good agreement.

Most of the objects observed in this way are hot Jupiters, but essentially the same method has also been applied to the 
Table 2. High-Resolution Cross Correlation Detections

\begin{tabular}{|c|c|c|c|c|}
\hline Planet & $\begin{array}{c}\text { Species } \\
\text { Detected }\end{array}$ & $\begin{array}{c}K_{P} \\
\left(\mathrm{~km} \mathrm{~s}^{-1}\right)\end{array}$ & $\begin{array}{c}M_{P} \\
\left(M_{J u p}\right)\end{array}$ & Reference \\
\hline HD $209458 b^{a}$ & $\operatorname{CO} 5.6 \sigma$ & $140 \pm 10$ & $0.64 \pm 0.09$ & Snellen et al. (2010a) \\
\hline$\tau$ Boo b & $\mathrm{CO} 6.2 \sigma$ & $110 \pm 3.2$ & $5.95 \pm 0.28$ & Brogi et al. (2012) \\
\hline$\tau$ Boo b & $\mathrm{CO} 3.4 \sigma$ & $115 \pm 11$ & $5.6 \pm 0.7$ & Rodler et al. (2012) \\
\hline$\tau$ Boo b & $\mathrm{H}_{2} \mathrm{O} 6 \sigma$ & $111 \pm 5$ & $5.9_{-0.20}^{+0.35}$ & Lockwood et al. (2014) \\
\hline 51 Peg b & $\mathrm{CO} / \mathrm{H}_{2} \mathrm{O} 5.9 \sigma$ & $134.1 \pm 1.8$ & $0.46 \pm 0.02$ & Brogi et al. (2013) \\
\hline HD 189733b & $\operatorname{CO} 5.0 \sigma$ & $154_{-3}^{+4}$ & $1.162_{-0.039}^{+0.058}$ & de Kok et al. (2013) \\
\hline HD $189733 b$ & $\mathrm{H}_{2} \mathrm{O} 4.8 \sigma$ & $154_{-10}^{+14}$ & & Birkby et al. (2013) \\
\hline HD 189733b & $\mathrm{CO} 3.4 \sigma$ & 154 & & Rodler, Kürster, \& Barnes (2013) \\
\hline HD 179949b & $\mathrm{CO} / \mathrm{H}_{2} \mathrm{O} 6.3 \sigma$ & $142.8 \pm 3.4$ & $0.98 \pm 0.04$ & Brogi et al. (2014) \\
\hline$\beta$ Pic b & $\mathrm{CO} 6.4 \sigma$ & & & Snellen et al. (2014) \\
\hline
\end{tabular}

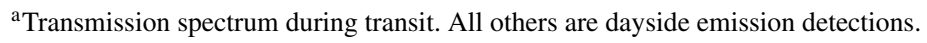

directly imaged exoplanet $\beta$ Pic $\mathrm{b}$ (Snellen et al. 2014). In this case it was possible to detect rotational broadening of about $25 \mathrm{kms}^{-1}$ in the $\mathrm{CO}$ cross correlation signal indicating a rapid rotation for the planet.

All detections so far are either for carbon monoxide or water. In HD189733b (de Kok et al. 2013) $\mathrm{CO}_{2}, \mathrm{CH}_{4}$ and $\mathrm{H}_{2} \mathrm{O}$ were searched for in the $2 \mu \mathrm{m}$ region but not detected. However, $\mathrm{H}_{2} \mathrm{O}$ was detected in HD $189733 \mathrm{~b}$ using longer wavelength $(3.2 \mu \mathrm{m})$ observations (Birkby et al. 2013). While CO is expected to be strong feature in these planets, part of the reason it is most easily detected may be that as a diatomic molecule it has a simpler spectrum and better quality line lists. Difficulty in detecting other species may, in part, be due to errors in the template spectra due to problems with the line lists, such as errors in line positions (see discussion in Barnes et al. 2010) and incompleteness. Methane line lists used for atmospheric modelling are known to be missing many hot bands that are needed at the high temperatures of these objects.

\subsubsection{Secondary eclipse photometry and spectroscopy}

The secondary eclipse (or occultation ${ }^{2}$ ) occurs when a planet passes behind the star. If the planet is sufficiently bright a measurable dip in the light curve is seen, and the fractional depth of the dip is a direct measurement of the flux from the planet as a fraction of that from the star. In most cases such measurements detect thermal emission from the dayside of the planet, and so contrasts are greatest at infrared wavelengths.

The first detection of a secondary eclipse was made at $24 \mu \mathrm{m}$ for HD 209458b using the Spitzer Space Telescope (Deming et al. 2005). Since then a substantial number of mostly hot Jupiter type systems have had their secondary

\footnotetext{
${ }^{2}$ Referring to this event as the secondary eclipse is consistent with standard terminology for eclipsing binary systems. The term occultation for this event is suggested by terminology used in the Solar system for e.g. the phenomena of Jupiter's satellites, where eclipses, occultations and transits all occur, and eclipse is reserved for the case where a satellite passes into the shadow of the planet. Both terms are used in the exoplanet literature with secondary eclipse being more common.
}

eclipse depth measured in the Spitzer/IRAC bands (3.6 $\mu \mathrm{m}$, $4.5 \mu \mathrm{m}, 5.8 \mu \mathrm{m}$ and $8.0 \mu \mathrm{m})$. There are also a number of measurements at shorter wavelengths from ground-based telescopes. The broad band eclipse depth results are summarised in Table 3. This lists secondary eclipse depths measured in the four Spitzer/IRAC bands and in the $\mathrm{K}_{s}$ band $(2.15 \mu \mathrm{m})$. Where there are multiple measurements in a band the one with the smaller quoted error is listed, but references to all measurements are given. The 'Other' column lists other bands in which eclipse depths have been measured and the references to these are also given.

For a few of the brighter systems it is possible to go further and obtain spectra of the dayside emission using the secondary eclipse depth. Such results are listed in Table 4. Figure 13 shows the combined data from broad band and spectroscopic observations for some of the best studied cases.

With secondary eclipse data of sufficient quality it is possible to map the brightness distribution across the disk of the planet (Williams et al. 2006). This has been attempted for HD 189733b by Majeau, Agol, \& Cowan (2012) and de Wit et al. (2012). The results show a bright spot shifted east from the subsolar point in agreement with results from the full phase light curve (Knutson et al. 2007a)

While the infrared secondary eclipse shows the thermal emission from the planet, observations of the secondary eclipse at visible wavelengths can show the planet through light reflected from its star. However, if the planet is very hot, thermal emission may still be present even at visible wavelengths. Table 5 summarises measurements so far, mostly from observations with Kepler, in a broad band covering $400-850 \mathrm{~nm}$. These observations provide a measure of the geometric albedo of the planet, and show that some of these planets are quite dark, while others have geometric albedos up to $\sim 0.4$. In the case of HD $189733 \mathrm{~b}$ observations have been made with STIS showing the planet to be dark at 450$570 \mathrm{~nm}$, but with an albedo of 0.4 at $290-450 \mathrm{~nm}$, the blue colour being indicative of a Rayleigh scattering haze (Evans 
Table 3. Secondary eclipse depth broadband measurements (\%)

\begin{tabular}{|c|c|c|c|c|c|c|c|}
\hline Planet & $\mathrm{K}_{s}$ & $3.6 \mu \mathrm{m}$ & $4.5 \mu \mathrm{m}$ & $5.8 \mu \mathrm{m}$ & $8.0 \mu \mathrm{m}$ & Other & References \\
\hline CoRoT-1b & $0.336 \pm .042$ & $0.415 \pm .042$ & $0.482 \pm .042$ & & & $0.71 \mu \mathrm{m}$ & $1,2,3,4$ \\
\hline CoRoT-2b & & $0.355 \pm .020$ & $0.500 \pm .020$ & & $0.510 \pm .059$ & $0.71 \mu \mathrm{m}$ & $5,6,7$ \\
\hline GJ436b & & $0.041 \pm .003$ & $<0.010$ & $0.033 \pm .014$ & $0.054 \pm .008$ & $16,24 \mu \mathrm{m}$ & 8,58 \\
\hline HD 149026b & & $0.040 \pm .003$ & $0.034 \pm .006$ & $0.044 \pm .010$ & $0.052 \pm .006$ & $16 \mu \mathrm{m}$ & 9 \\
\hline HD 189733b & & $0.147 \pm .004$ & $0.179 \pm .004$ & $0.310 \pm .034$ & $0.344 \pm .004$ & $16,24 \mu \mathrm{m}$ & $10,11,12,59$ \\
\hline HD 209458b & & $0.094 \pm .009$ & $0.139 \pm .007$ & $0.301 \pm .043$ & $0.240 \pm .026$ & $24 \mu \mathrm{m}$ & $13,14,15,62$ \\
\hline HAT-P-1b & $0.109 \pm .025$ & $0.080 \pm .008$ & $0.135 \pm .022$ & $0.203 \pm .031$ & $0.238 \pm .040$ & & 16,17 \\
\hline HAT-P-3b & & $0.112_{-.030}^{+.015}$ & $0.094_{-.009}^{+.016}$ & & & & 18 \\
\hline HAT-P-4b & & $0.142_{-.016}^{+.014}$ & $0.122_{-.014}^{+.0099}$ & & & & 18 \\
\hline HAT-P-6b & & $0.117 \pm .008$ & $0.106 \pm .006$ & & & & 19 \\
\hline HAT-P-7b & & $0.098 \pm .017$ & $0.159 \pm .022$ & $0.245 \pm .031$ & $0.225 \pm .052$ & & 20 \\
\hline HAT-P-8b & & $0.131_{-.010}^{+.007}$ & $0.111_{-.007}^{+.008}$ & & & & 19 \\
\hline HAT-P-23b & $0.234 \pm .046$ & $0.248 \pm .019$ & $0.309 \pm .026$ & & & & 60 \\
\hline Kepler-5b & & $0.103 \pm .017$ & $0.107 \pm .015$ & & & & 21 \\
\hline Kepler-6b & & $0.069 \pm .027$ & $0.151 \pm .019$ & & & & 21 \\
\hline Kepler-12b & & $0.137 \pm .020$ & $0.116 \pm .031$ & & & & 22 \\
\hline TrES-1b & & & $0.066 \pm .013$ & & $0.225 \pm .036$ & & 23 \\
\hline TrES-2b & $0.062_{-011}^{+.013}$ & $0.127 \pm .021$ & $0.230 \pm .024$ & $0.199 \pm .054$ & $0.359 \pm .060$ & & 24,25 \\
\hline TrES-3b & $0.133_{-0.016}^{+0.018}$ & $0.346 \pm .035$ & $0.372 \pm .054$ & $0.449 \pm .097$ & $0.475 \pm .046$ & & $26,27,28$ \\
\hline TrES-4b & & $0.137 \pm .011$ & $0.148 \pm .016$ & $0.261 \pm .059$ & $0.318 \pm .044$ & & 29 \\
\hline WASP-3b & $0.181 \pm .020$ & $0.209_{-0.028}^{+0.040}$ & $0.282 \pm 0.012$ & & $0.328_{-0.055}^{+0.086}$ & & 30,64 \\
\hline WASP-4b & $0.182_{-.013}^{+.014}$ & $0.319 \pm .031$ & $0.343 \pm .027$ & & & & 31,32 \\
\hline WASP-5b & $0.269 \pm .062$ & $0.197 \pm .028$ & $0.227 \pm .025$ & & & $\mathrm{H}$ & 33,63 \\
\hline WASP-8b & & $0.113 \pm .018$ & $0.069 \pm .007$ & $0.093 \pm .023$ & & & 34 \\
\hline WASP-12b & $0.339 \pm .014^{a}$ & $0.419 \pm .014^{b}$ & $0.424 \pm .021^{b}$ & $0.694 \pm .057^{b}$ & $0.701 \pm .074^{b}$ & $\mathrm{z}, \mathrm{J}, \mathrm{H}, 2.3 \mu \mathrm{m}$ & $35,36,37,38,39,40$ \\
\hline WASP-14b & & $0.19 \pm 0.01$ & $0.224 \pm .018$ & & $0.181 \pm 0.022$ & & 61 \\
\hline WASP-17b & & & $0.229 \pm .013$ & $0.237 \pm .039$ & & & 41 \\
\hline WASP-18b & & $0.304 \pm .017$ & $0.379 \pm .008$ & $0.37 \pm .03$ & $0.41 \pm .02$ & & 42,43 \\
\hline WASP-19b & $0.287 \pm .020$ & $0.483 \pm .025$ & $0.572 \pm .030$ & $0.65 \pm .11$ & $0.73 \pm .12$ & $\mathrm{z}, \mathrm{H}$ & $44,45,46,47,57,67$ \\
\hline WASP-24b & & $0.159 \pm .013$ & $0.202 \pm .018$ & & & & 48 \\
\hline WASP-26b & & $0.126 \pm .013$ & $0.149 \pm .016$ & & & & 49 \\
\hline WASP-33b & $0.244_{-.020}^{+.027}$ & $0.26 \pm .05$ & $0.41 \pm .02$ & & & $\mathrm{z}$ & $50,51,52$ \\
\hline WASP-43b & $0.181 \pm .027$ & $0.346 \pm .013$ & $0.382 \pm .015$ & & & & $53,66,67$ \\
\hline WASP-46b & $0.253_{-.060}^{+.063}$ & & & & & & 65 \\
\hline WASP-48b & $0.109 \pm .027$ & $0.176 \pm .013$ & $0.214 \pm .020$ & & & & 60 \\
\hline $\mathrm{XO}-1 \mathrm{~b}$ & & $0.086 \pm .007$ & $0.122 \pm .009$ & $0.261 \pm .031$ & $0.210 \pm .029$ & & 54 \\
\hline $\mathrm{XO}-2 \mathrm{~b}$ & & $0.081 \pm .017$ & $0.098 \pm .020$ & $0.167 \pm .036$ & $0.133 \pm .049$ & & 55 \\
\hline $\mathrm{XO}-3 \mathrm{~b}$ & & $0.101 \pm .004$ & $0.143 \pm .006$ & $0.134 \pm .049$ & $0.150 \pm .036$ & & 56 \\
\hline $\mathrm{XO}-4 \mathrm{~b}$ & & $0.056_{-.006}^{+.012}$ & $0.135_{-.007}^{+.010}$ & & & & 19 \\
\hline
\end{tabular}

${ }^{a}$ Value from Croll et al. (2011) as corrected by Crossfield et al. (2012b)

bValues from Campo et al. (2011) as corrected by Crossfield et al. (2012b)

References 1. Deming et al. (2011), 2. Gillon et al. (2009), 3. Rogers et al. (2009), 4. Snellen et al. (2009), 5. Deming et al. (2011), 6. Gillon et al. (2010), 7. Snellen et al. (2010b), 8. Deming et al. (2007), 9. Stevenson et al. (2012), 10. Charbonneau et al. (2008), 11. Knutson et al. (2012), 12. Deming et al. (2006), 13. Deming et al. (2005), 14. Knutson et al. (2008), 15. Crossfield et al. (2012a), 16. Todorov et al. (2010), 17. de Mooij et al. (2011), 18. Todorov et al. (2013), 19. Todorov et al. (2012), 20 Christiansen et al. (2010), 21. Désert et al. (2011a), 22. Fortney et al. (2011), 23. Charbonneau et al. (2005), 24. O'Donovan et al. (2010), 25. Croll et al. (2010a), 26. Fressin et al. (2010), 27. de Mooij \& Snellen (2009), 28. Croll et al. (2010b), 29. Knutson et al. (2009a), 30. Zhao et al. (2012a), 31. Beerer et al. (2011), 32. Cáceres et al. (2011), 33. Baskin et al. (2013), 34. Cubillas et al. (2013), 35. Campo et al. (2011), 36. Croll et al. (2011), 37. Crossfield et al. (2012b), 38. Zhao et al. (2012b), 39. Cowan et al. (2012b), 40. López-Morales et al. (2010), 41. Anderson et al. (2011), 42. Nyemeyer et al. (2011), 43. Maxted et al. (2013), 44. Anderson et al. (2013), 45. Gibson et al. (2010), 46. Lendl et al. (2013), 47. Burton et al. (2012), 48. Smith et al. (2012), 49. Mahtani et al. (2013), 50. Deming et al. (2012), 51. de Mooij et al. (2013), 52. Smith et al. (2011), 53. Blecic et al. (2014), 54. Machalek et al. (2008), 55. Machalek et al. (2009), 56. Machalek et al. (2010), 57. Zhou et al. (2013), 58. Stevenson et al. (2010), 59. Agol et al. (2010), 60. O'Rourke et al. (2014), 61. Blecic et al. (2013), 62. Zellem et al. (2014), 63. Chen et al. (2014a), 64. Rostron et al. (2014), 65. Chen et al. (2014b), 66. Wang et al. (2013), 67. Zhou et al. (2014)

et al. 2013). Low albedos in the visible are to be expected for clear atmospheres due to the broad sodium and absorption lines, whereas higher albedos can result if clouds are present (Sudarsky, Burrows, \& Pinto 2000).

\subsubsection{Transit spectroscopy}

Observations during transit (or primary eclipse, when the planet passes in front of the star) also provide information on the atmospheres. The depth of the primary eclipse is a 
Table 4. Dayside emission spectroscopy from secondary eclipses.

\begin{tabular}{|c|c|c|c|c|}
\hline Planet & Wavelengths & Instrument & Features Reported & Reference \\
\hline CoRoT-2b & $1.1-1.7 \mu \mathrm{m}$ & HST/WFC3 & & Wilkins et al. (2014) \\
\hline \multirow[t]{6}{*}{ HD 189733b } & $5-14 \mu \mathrm{m}$ & Spitzer/IRS & $\mathrm{H}_{2} \mathrm{O}$ & Grillmair et al. (2008) \\
\hline & $1.5-2.5 \mu \mathrm{m}$ & HST/NICMOS & $\mathrm{H}_{2} \mathrm{O}, \mathrm{CO}, \mathrm{CO}_{2}$ & Swain et al. (2009a) \\
\hline & $2.0-4.1 \mu \mathrm{m}$ & IRTF/SpeX & Emission feature at $3.3 \mu \mathrm{m}$ & Swain et al. (2010) \\
\hline & $3.27-3.31 \mu \mathrm{m}$ & Keck/NIRSPEC & No emission feature at $3.3 \mu \mathrm{m}$ & Mandell et al. (2011) \\
\hline & $2.0-4.1 \mu \mathrm{m}$ & IRTF/SpeX & Confirm emission at $3.3 \mu \mathrm{m}$ & Waldmann et al. (2012) \\
\hline & $1.1-1.7 \mu \mathrm{m}$ & HST/WFC3 & $\mathrm{H}_{2} \mathrm{O}$ & Crouzet et al. (2014) \\
\hline \multirow[t]{2}{*}{ HD 209458b } & $7.5-13.2 \mu \mathrm{m}$ & Spitzer/IRS & & Richardson et al. (2007) \\
\hline & $1.5-2.5 \mu \mathrm{m}$ & HST/NICMOS & $\mathrm{H}_{2} \mathrm{O}, \mathrm{CH}_{4}, \mathrm{CO}_{2}$ & Swain et al. (2009b) \\
\hline TrES-3b & $1.1-1.7 \mu \mathrm{m}$ & HST/WFC3 & & Ranjan et al. (2014) \\
\hline WASP-4b & $1.1-1.7 \mu \mathrm{m}$ & HST/WFC3 & & Ranjan et al. (2014) \\
\hline \multirow[t]{2}{*}{ WASP-12b } & $1-2.5 \mu \mathrm{m}$ & IRTF/SpeX & & Crossfield et al. (2012c) \\
\hline & $1.1-1.7 \mu \mathrm{m}$ & HST/WFC3 & & Swain et al. (2013) \\
\hline WASP-19b & $1.25-2.35$ & Magellan/MMIRS & & Bean et al. (2013) \\
\hline WASP-43b & $1.1-1.7 \mu \mathrm{m}$ & HST/WFC3 & $\mathrm{H}_{2} \mathrm{O}$ & Kreidberg et al. (2014b) \\
\hline
\end{tabular}

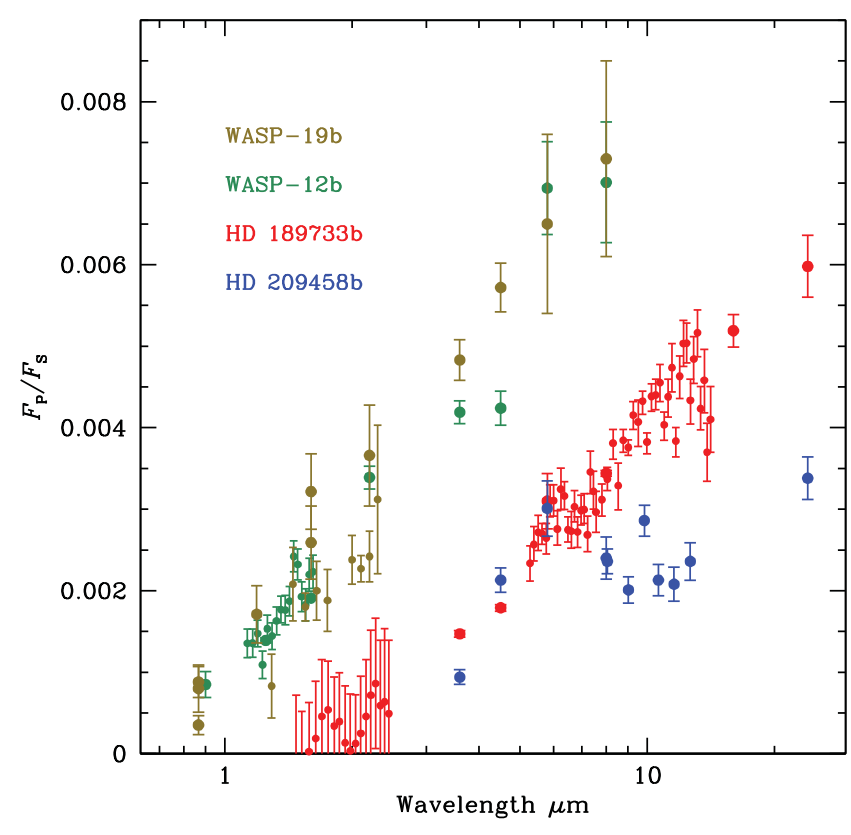

Figure 13. Dayside emission from WASP-12b, WASP-19b, HD189733b and HD209458b based on data listed in Tables 3 and 4. Large symbols are broad band measurements and smaller symbols are spectroscopic observations.

measure of the radius of the planet, and will be larger where absorption is strongest.

Spectroscopy during transits can reveal absorption features in the transmission spectrum of the planet's atmosphere. Transit spectroscopy samples the terminator of the planet and the long tangent path length means that it is sensitive to higher levels in the atmosphere than dayside emission spectroscopy from secondary eclipses.

The first detection of an exoplanet atmosphere was in observations of the transits of HD 209458 (Charbonneau et al. 2002) that showed absorption in the sodium doublet at

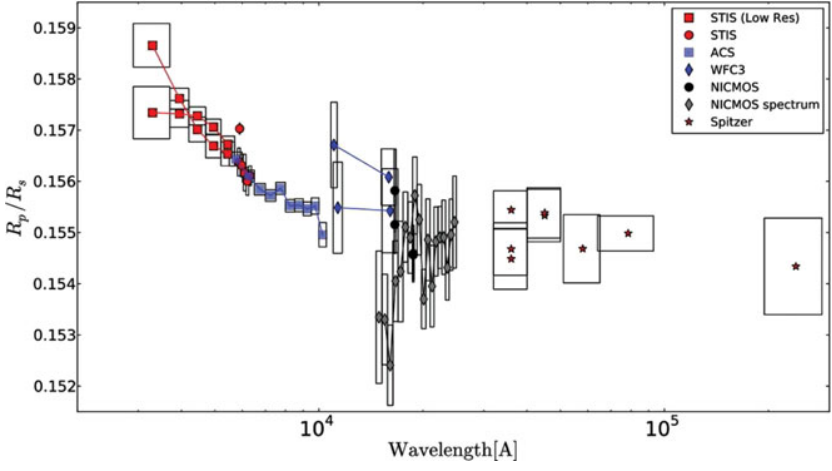

Figure 14. Transmission spectrum of HD 189733b from HST and Spitzer transit observations, showing increase to the blue interpreted as due to a Rayleigh scattering haze. - Figure 9 from 'The prevalence of dust on the exoplanet HD189733b from Hubble and Spitzer observations', Pont, F. et al., 2013, MNRAS, 432, 2917.

$589.3 \mathrm{~nm}$. Transit spectroscopy (other than studies of $\mathrm{Na}$ line absorption) studies are listed in Table 6. In addition to these spectroscopy observations there are numerous transit measurements in broad band filters, including measurements in the Spitzer/IRAC bands that extend coverage to longer wavelengths. HD 189733b is a particularly well studied system, and the various space observations have been combined by Pont et al. (2013) to give the transmission spectrum shown in Figure 14. It shows increasing absorption to the blue indicating the presence of a Rayleigh scattering haze. Wasp $12 \mathrm{~b}$ (Sing et al. 2013) also shows a similar increase to the blue attributed to Rayleigh scattering from aerosols.

Transmission spectra in the near-IR for three systems are shown in Figure 15, showing water vapour absorption at $\sim 1.4 \mu \mathrm{m}$. As is conventional, these observations are plotted as $\mathrm{R}_{P} / \mathrm{R}_{S}$ (i.e. the radius of the planet divided by the radius of the star as determined from the transit). This gives an inverted spectrum compared with conventional spectroscopy, 
Table 5. Geometric albedo measurements from optical secondary eclipses.

\begin{tabular}{|c|c|c|c|}
\hline Planet & $\begin{array}{l}\text { Secondary eclipse } \\
\text { depth (ppm) }\end{array}$ & $\begin{array}{l}\text { Geometric } \\
\text { albedo }\end{array}$ & Reference \\
\hline HD 189733b (290-450 nm) & $126_{-36}^{+37}$ & $0.40_{-0.11}^{+0.12}$ & Evans et al. (2013) \\
\hline HD 189733b (450-570 nm) & $1_{-30}^{+3 \overline{7}}$ & $0.00_{-0.10}^{+0.11}$ & Evans et al. (2013) \\
\hline HD 209458b & $7 \pm 9$ & $0.038 \pm 0.045$ & Rowe et al. (2008) \\
\hline \multirow[t]{3}{*}{ Kepler-5b } & $21 \pm 6$ & $0.12 \pm 0.04$ & Désert et al. (2011a) \\
\hline & $19 \pm 4$ & $0.12 \pm 0.02$ & Esteves et al. (2013) \\
\hline & $19.8 \pm 3.65$ & $0.16 \pm 0.03$ & Angerhausen et al. (2014) \\
\hline \multirow[t]{3}{*}{ Kepler-6b } & $22 \pm 7$ & $0.11 \pm 0.04$ & Désert et al. (2011a) \\
\hline & $8.9 \pm 3.8$ & $0.059 \pm 0.025$ & Esteves et al. (2013) \\
\hline & $11.3 \pm 4.2$ & $0.07 \pm 0.03$ & Angerhausen et al. (2014) \\
\hline \multirow[t]{4}{*}{ Kepler-7b } & $47 \pm 14$ & $0.38 \pm 0.12$ & Kipping \& Bakos (2011) \\
\hline & $42 \pm 4$ & $0.32 \pm 0.03$ & Demory et al. (2011) \\
\hline & $48 \pm 3$ & $0.35 \pm 0.02$ & Demory et al. (2013) \\
\hline & $46.6 \pm 4.0$ & $0.32 \pm 0.03$ & Angerhausen et al. (2014) \\
\hline \multirow{2}{*}{ Kepler-8b } & $26 \pm 6$ & $0.14 \pm 0.03$ & Esteves et al. (2013) \\
\hline & $16.5 \pm 4.45$ & $0.11 \pm 0.03$ & Angerhausen et al. (2014) \\
\hline \multirow[t]{2}{*}{ Kepler-12b } & $31 \pm 7$ & $0.14 \pm 0.04$ & Fortney et al. (2011) \\
\hline & $18.7 \pm 4.9$ & $0.09 \pm 0.02$ & Angerhausen et al. (2014) \\
\hline Kepler-13Ab & $173.7 \pm 1.8$ & $0.33_{-0.06}^{+0.04}$ & Shporer et al. (2014) \\
\hline \multirow[t]{2}{*}{ Kepler-17b } & $58 \pm 10$ & $0.10 \pm 0.02$ & Désert et al. (2011b) \\
\hline & $43.7 \pm 6.4$ & $0.08 \pm 0.01$ & Angerhausen et al. (2014) \\
\hline \multirow[t]{3}{*}{ Kepler-41b } & $64_{-12}^{+10}$ & $0.30 \pm 0.08$ & Santerne et al. (2011) \\
\hline & $60 \pm 9$ & $0.23 \pm 0.05$ & Quintana et al. (2013) \\
\hline & $46.2 \pm 8.7$ & $0.18 \pm 0.03$ & Angerhausen et al. (2014) \\
\hline Kepler-43b & $17.0 \pm 5.3$ & $0.06 \pm 0.02$ & Angerhausen et al. (2014) \\
\hline Kepler-76b & $75.6 \pm 5.6$ & $0.22 \pm 0.02$ & Angerhausen et al. (2014) \\
\hline \multirow[t]{2}{*}{ Kepler-412b } & $47.4 \pm 7.4$ & $0.094 \pm 0.015$ & Deleuil et al. (2014) \\
\hline & $40.2 \pm 9.0$ & $0.11 \pm 0.02$ & Angerhausen et al. (2014) \\
\hline \multirow[t]{2}{*}{ TrES-2b } & $6.5 \pm 1.9$ & $0.025 \pm 0.007$ & Kipping \& Spiegel (2011) \\
\hline & $7.5 \pm 1.7$ & $0.030 \pm 0.007$ & Esteves et al. (2013) \\
\hline
\end{tabular}

All measurements are with Kepler (400-850 nm) except for HD 209458b observed with MOST (350-700 nm) and HD 189733 b observed with HST/STIS.

since absorption features increase the apparent radius of the planet.

Measurements of the Sodium D-line absorption are listed in Table 7. Results are listed here where the absorption is detected at greater than the 3-sigma level. There are also a number of unsuccesful attempts at detections. Potassium absorption has been reported in XO-2b (Sing et al. 2011b).

Atomic and atomic ion species have also been detected in a number of transiting planets in the unbound portion of the atmosphere, or exosphere. The best studied case is HD 209458b where H I, C II, O I, and Si III have been observed (Vidal-Madjar et al. 2003, 2004; Linsky et al. 2010). Exosphere detections have also been reported in HD 189733b (Lecavelier Des Etangs et al. 2010; Jensen et al. 2012) and Wasp-12b (Fossati et al. 2010).

\subsubsection{Full phase photometry}

As well as observations of the transits and eclipses, information on a planet's atmosphere can be obtained from observations of the full phase light curve. In the infrared a hot Jupiter will show variations around the cycle due to the variation of temperature across its surface. In the optical where reflected light is seen, variations will occur due to the change in the illuminated fraction of the disk, as well as due to phase angle dependent scattering processes (Seager, Whitney, \& Sasselov 2000). In some cases the light curves are complicated by ellipsoidal variations in the star (e.g. Welsh et al. 2010) or the planet (e.g. Cowan et al. 2012b). Systems with full phase light curves at infrared wavelengths showing significant variation around the cycle are listed in Table 8. In addition full phase light curves due to reflected light are observed in many of the systems listed in Table 5.

Analysis of these light curves has been used to derive maps of the temperature distribution of HD 189733b (Knutson et al. 2007a) showing a hot spot offset from the substellar point (consistent with models, see Section 4.1.3). In the case of Kepler-7b, the reflected light phase curve observed by Kepler is interpreted as showing the presence of patchy clouds (Demory et al. 2013).

\subsubsection{Polarimetry}

Reflected light from extrasolar planets will be polarised as a result of scattering from cloud and haze particles and from molecules. Normal stars are generally found to have very 
Table 6. Transmission spectroscopy during transit.

\begin{tabular}{|c|c|c|c|c|}
\hline Planet & Wavelengths & Instrument & Featues reported & Reference \\
\hline \multirow[t]{2}{*}{ CoRoT-1b } & $0.8-2.4 \mu \mathrm{m}$ & IRTF/SpeX/MORIS & \multirow[t]{6}{*}{ no TiO/VO } & Schlawin et al. (2014) \\
\hline & $1.1-1.7 \mu \mathrm{m}$ & HST/WFC3 & & Ranjan et al. (2014) \\
\hline GJ 436b & $1.1-1.7 \mu \mathrm{m}$ & HST/WFC3 & & Knutson et al. (2014a) \\
\hline \multirow[t]{4}{*}{ GJ $1214 b$} & $0.78-1.00 \mu \mathrm{m}$ & VLT/FORS1 & & Bean, Miller-Ricci Kempton, \& Homeier (2010) \\
\hline & $2.1-2.4 \mu \mathrm{m}$ & Keck/NIRSPEC & & Crossfield et al. (2011) \\
\hline & $1.1-1.7 \mu \mathrm{m}$ & HST/WFC3 & & Berta et al. (2012) \\
\hline & $1.1-1.7 \mu \mathrm{m}$ & HST/WFC3 & \multirow[t]{4}{*}{ clouds } & Kreidberg et al. (2014a) \\
\hline \multirow[t]{2}{*}{ GJ 3470b } & $2.09-2.36 \mu \mathrm{m}$ & Keck/MOSFIRE & & Crossfield et al. (2013) \\
\hline & $1.1-1.7 \mu \mathrm{m}$ & HST/WFC3 & & Ehrenreich et al. (2014) \\
\hline HD 97658b & $1.1-1.7 \mu \mathrm{m}$ & HST/WFC3 & & Knutson et al. (2014b) \\
\hline \multirow[t]{6}{*}{ HD 189733b } & $1.4-2.5 \mu \mathrm{m}$ & HST/NICMOS & \multirow{5}{*}{$\begin{array}{l}\mathrm{H}_{2} \mathrm{O}, \mathrm{CH}_{4} \\
\text { haze } \\
\text { haze, no } \mathrm{H}_{2} \mathrm{O} \\
\text { haze }\end{array}$} & Swain et al. (2008) \\
\hline & $0.55-1.05 \mu \mathrm{m}$ & $\mathrm{HST} / \mathrm{ACS}$ & & Pont et al. (2008) \\
\hline & $1.66-1.87 \mu \mathrm{m}$ & HST/NICMOS & & Sing et al. (2009) \\
\hline & $0.29-0.57 \mu \mathrm{m}$ & HST/STIS & & Sing et al. (2011a) \\
\hline & $1.1-1.7 \mu \mathrm{m}$ & HST/WFC3 & & Gibson et al. (2012) \\
\hline & $1.1-1.7 \mu \mathrm{m}$ & HST/WFC3 & $\mathrm{H}_{2} \mathrm{O}$ & McCullough et al. (2014) \\
\hline \multirow[t]{2}{*}{ HD 209458b } & $0.3-1.0 \mu \mathrm{m}$ & HST/STIS & $\mathrm{H}_{2}^{2} \mathrm{O}^{a}$ & Knutson et al. (2007b) \\
\hline & $1.1-1.7 \mu \mathrm{m}$ & $\mathrm{HST} / \mathrm{WFC} 3$ & $\mathrm{H}_{2} \mathrm{O}$ & Deming et al. (2013) \\
\hline HAT-P-1b & $1.1-1.7 \mu \mathrm{m}$ & HST/WFC3 & $\mathrm{H}_{2}^{2} \mathrm{O}$ & Wakeford et al. (2013) \\
\hline \multirow[t]{2}{*}{ HAT-P-12b } & $1.1-1.7 \mu \mathrm{m}$ & HST/WFC3 & \multirow[t]{7}{*}{ clouds } & Line et al. (2013a) \\
\hline & $0.52-0.93 \mu \mathrm{m}$ & Gemini/GMOS & & Gibson et al. (2013a) \\
\hline TrES-2b & $1.1-1.7 \mu \mathrm{m}$ & HST/WFC3 & & Ranjan et al. (2014) \\
\hline TrES-4b & $1.1-1.7 \mu \mathrm{m}$ & HST/WFC3 & & Ranjan et al. (2014) \\
\hline WASP-6b & $0.48-0.86 \mu \mathrm{m}$ & Baade/IMACS & & Jordán et al. (2013) \\
\hline \multirow[t]{4}{*}{ WASP-12b } & $1.1-1.7 \mu \mathrm{m}$ & HST/WFC3 & & Swain et al. (2013) \\
\hline & $0.7-1.0 \mu \mathrm{m}$ & Gemini/GMOS & & Stevenson et al. (2014) \\
\hline & $0.3-1.7 \mu \mathrm{m}$ & HST/STIS+WFC3 & \multirow[t]{4}{*}{ aerosols, no $\mathrm{TiO}$} & Sing et al. (2013) \\
\hline & $1.1-1.7 \mu \mathrm{m}$ & HST/WFC3 & & Mandell et al. (2013) \\
\hline WASP-17b & $1.1-1.7 \mu \mathrm{m}$ & HST/WFC3 & & Mandell et al. (2013) \\
\hline \multirow[t]{3}{*}{ WASP-19b } & $1.25-2.35 \mu \mathrm{m}$ & Magellan/MMIRS & & Bean et al. (2013) \\
\hline & $0.29-1.69 \mu \mathrm{m}$ & HST/STIS+WFC3 & \multirow{2}{*}{$\mathrm{H}_{2} \mathrm{O}$ no $\mathrm{TiO}$} & Huitson et al. (2013) \\
\hline & $1.1-1.7 \mu \mathrm{m}$ & HST/WFC3 & & Mandell et al. (2013) \\
\hline WASP-29b & $0.51-0.72 \mu \mathrm{m}$ & Gemini/GMOS & & Gibson et al. (2013b) \\
\hline \multirow[t]{2}{*}{ WASP-43b } & $0.52-1.04 \mu \mathrm{m}$ & GTC/OSIRIS & \multirow{5}{*}{$\begin{array}{l}\mathrm{H}_{2} \mathrm{O} \\
\mathrm{H}_{2} \mathrm{O}, \mathrm{CO}_{2}, \mathrm{CH}_{4} \\
\mathrm{H}_{2} \mathrm{O}\end{array}$} & Murgas et al. (2014) \\
\hline & $1.1-1.7 \mu \mathrm{m}$ & HST/WFC3 & & Kreidberg et al. (2014b) \\
\hline \multirow[t]{2}{*}{$\mathrm{XO}-1 \mathrm{~b}$} & $1.2-1.8 \mu \mathrm{m}$ & HST/NICMOS & & Tinetti et al. (2010) \\
\hline & $1.1-1.7 \mu \mathrm{m}$ & HST/WFC3 & & Deming et al. (2013) \\
\hline $\mathrm{XO}-2 \mathrm{~b}$ & $1.1-1.7 \mu \mathrm{m}$ & HST/NICMOS & & Crouzet et al. (2012) \\
\hline
\end{tabular}

${ }^{a}$ According to reanalysis by Barman (2007).

Table 7. Sodium (589 $\mathrm{nm}$ ) detections from transit spectroscopy.

\begin{tabular}{lllll}
\hline \hline Planet & \multicolumn{1}{c}{ Instrument } & \multicolumn{1}{c}{ Line depth } & \multicolumn{1}{c}{ Band } & \multicolumn{1}{c}{ Reference } \\
\hline HD 189733b & HET/HRS & $6.72 \pm 2.07 \times 10^{-4}$ & $1.2 \mathrm{~nm}$ & Redfield et al. (2008) \\
& HET/HRS & $5.26 \pm 1.69 \times 10^{-4}$ & $1.2 \mathrm{~nm}$ & Jensen et al. (2011) \\
& HST/STIS & $9 \pm 1 \times 10^{-4}$ & $0.5 \mathrm{~nm}$ & Huitson et al. (2012) \\
HD 209458b b & HST/STIS & $2.32 \pm 0.57 \times 10^{-4}$ & $1.2 \mathrm{~nm}$ & Charbonneau et al. (2002) \\
& HET/HRS & $2.63 \pm 0.81 \times 10^{-4}$ & $1.2 \mathrm{~nm}$ & Jensen et al. (2011) \\
& Subaru/HDS & $5.6 \pm 0.7^{a} \times 10^{-4}$ & $0.3 \mathrm{~nm}$ & Snellen et al. (2008) \\
HAT-P-1b & HST/STIS & $11 \times 10^{-4}$ & $0.44 \mathrm{~nm}$ & Sing et al. (2008) \\
WASP-17b & HST/STIS & $9.8 \pm 3.0 \times 10^{-4}$ & $3.0 \mathrm{~nm}$ & Nikolov et al. (2014) \\
& Magellan/MIKE & $55 \pm 13 \times 10^{-4}$ & $0.15 \mathrm{~nm}$ & Wood et al. (2011) \\
XO-2b & GTC/OSIRIS & $4.7 \pm 1.1 \times 10^{-4}$ & $0.15 \mathrm{~nm}$ & Zhou \& Bayliss (2012) \\
\hline \hline
\end{tabular}

a $7.0 \pm 1.1 \times 10^{-4}(0.15 \mathrm{~nm}$ band $), 13.5 \pm 1.7 \times 10^{-4}(0.075 \mathrm{~nm}$ band $)$ 
Table 8. Full phase infrared photometry of exoplanets.

\begin{tabular}{llcl}
\hline \hline Planet & Wavelength & Amplitude (ppm) & \multicolumn{1}{c}{ Reference } \\
\hline CoRoT-1b & $710 \mathrm{~nm}$ & $126 \pm 33$ & Snellen et al. (2007) \\
$v$ And b & $24 \mu \mathrm{m}$ & $1300 \pm 74$ & Crossfield et al. (2010) \\
HD 179949 & $8 \mu \mathrm{m}$ & $1410 \pm 330$ & Cowan, Agol, \& Charbonneau (2007) \\
HD 149026b & $8 \mu \mathrm{m}$ & $227 \pm 66$ & Knutson et al. (2009c) \\
HD 189733b & $3.6 \mu \mathrm{m}$ & $1242 \pm 61$ & Knutson et al. (2012) \\
& $4.5 \mu \mathrm{m}$ & $982 \pm 89$ & Knutson et al. (2012) \\
& $8 \mu \mathrm{m}$ & $1200 \pm 200$ & Knutson et al. (2007a) \\
HD 209458b & $4.5 \mu \mathrm{m}$ & $1090 \pm 120$ & Zellem et al. (2014) \\
HAT-P-7b & $400-850 \mathrm{~nm}$ & 63.7 & Welsh et al. (2010) \\
WASP-18b & $3.6 \mu \mathrm{m}$ & $2960 \pm 90$ & Maxted et al. (2013) \\
& $4.5 \mu \mathrm{m}$ & $3660 \pm 70$ & Maxted et al. (2013) \\
\hline \hline
\end{tabular}

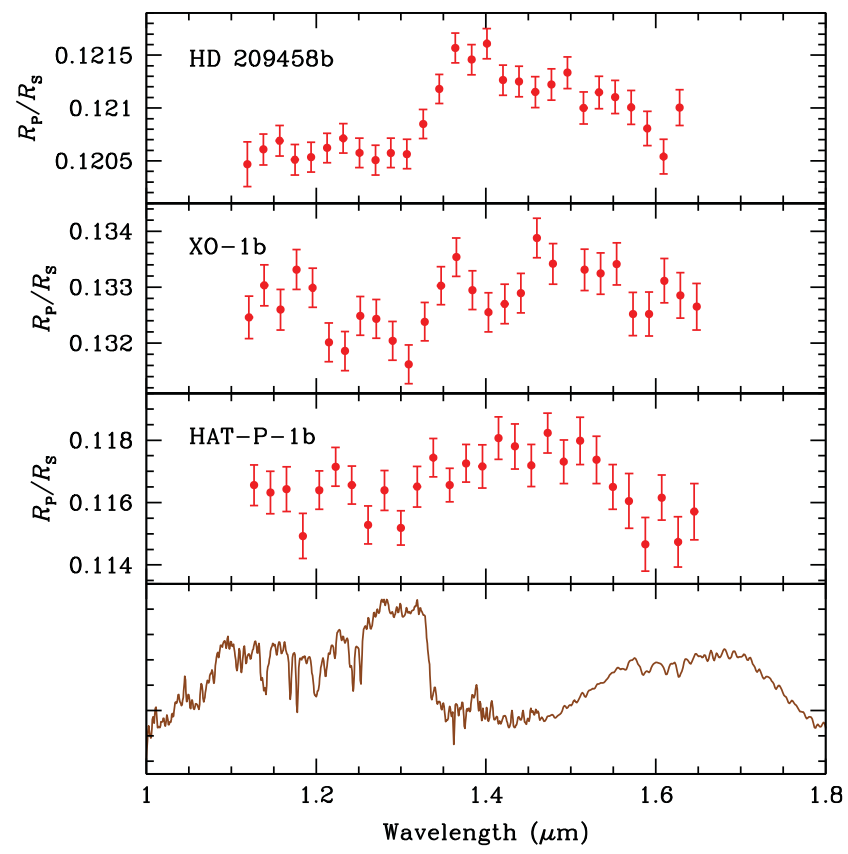

Figure 15. HST/WFC3 observations of the transmission spectra of HD209458b and XO-1b (Deming et al. 2013) and HAT-P-1b (Wakeford et al. 2013) showing absorption at $\sim 1.4 \mu \mathrm{m}$ attributed to $\mathrm{H}_{2} \mathrm{O}$. The spectrum of the brown dwarf Kelu-1 showing the same absorption feature is shown for comparison in the bottom panel. The exoplanet spectra are inverted compared with the brown dwarf spectra since absorption features increase the radius of the planet as seen in transit observations.

low intrinsic polarisations (Kemp et al. 1987; Bailey, Lucas, \& Hough 2010). In a hot Jupiter system the polarisation in the combined light of the unresolved star and planet is expected to be in the range $10^{-5}-10^{-6}$ (Seager, Whitney, \& Sasselov 2000), and will vary around the orbital cycle with the changing phase angle.

While the expected polarisations are small, polarisation is a differential measurement that can be made to high sensitivity wth ground-based telescopes, and instruments capable of measuring stellar polarisation at the one part per million level have been developed (Hough et al. 2006; Wiktorowicz
\& Matthews 2008). Lucas et al. (2009) reported upper limits on the polarisation of $\tau$ Boo and $55 \mathrm{Cnc}$ in a broad red band (590-920 nm) and set upper limits on the geometric albedo of $\tau$ Boo b and 55 Cnc e for Rayleigh scattering models.

Berdyugina et al. (2008) reported polarisation varying over the orbital cycle of HD 189733 b with an amplitude of $\sim 2 \times 10^{-4}$ in the B band. Wiktorowicz (2009), however, found no polarisation variation in this system with a $99 \%$ confidence upper limit of $7.9 \times 10^{-5}$ in a 400-675 nm wavelength range. Berdyugina et al. (2011) then reported further observations that confirmed a polarisation variation, but with a reduced amplitude of $10^{-4}$ in the $U$ and $B$ bands and much lower amplitude in the $\mathrm{V}$ band. They claim the data is consistent with that of Wiktorowicz (2009) when the different wavelengths are taken into account.

While HD 189733b is a system in which polarisation might be expected in view of the Rayleigh scattering haze seen in transmission spectroscopy (Pont et al. 2008, 2013), the reported polarisation amplitudes are too large to be easily explained. Berdyugina et al. (2011) report that the polarisation is consistent with a Rayleigh-Lambert model with a geometric albedo of $\sim 0.6$ and 'scattered light maximally polarised' (i.e. 100\%). However, in Rayleigh scattering models a layer sufficiently optically thick to produce a high geometric albedo has a maximum polarisation of only about $30 \%$ (Buenzli \& Schmid 2009) as a result of depolarisation due to multiple scattering. Lucas et al. (2009) used Monte-Carlo scattering models to predict a maximum polarisation amplitude of $2.6 \times 10^{-5}$ for HD $189733 \mathrm{~b}$.

\subsection{Atmospheric structure}

\subsubsection{Inflated atmospheres}

One result of transit observations is that many hot Jupiters are 'inflated', with radii significantly larger than predicted by models (Baraffe, Chabrier, \& Barman 2008, 2010). This inflation is found to be correlated with the level of stellar irradiation, with inflation becoming apparent for planets receiving incident flux greater than $2 \times 10^{8} \mathrm{erg} \mathrm{s}^{-1} \mathrm{~cm}^{-2}$ (Miller \& Fortney 2011; Demory \& Seager 2011). 
Weiss et al. (2013) have used data on 138 exoplanets to derive empirical relations between radius, mass and incident flux as follows:

$$
\begin{aligned}
& \qquad R_{P} / R_{\oplus}=1.78\left(M_{P} / M_{\oplus}\right)^{0.53}\left(F / \mathrm{erg} \mathrm{s}^{-1} \mathrm{~cm}^{-2}\right)^{-0.03} \\
& \text { for } M_{P}<150 M_{\oplus} \text {, and } \\
& \qquad R_{P} / R_{\oplus}=2.45\left(M_{P} / M_{\oplus}\right)^{-0.039}\left(F / \mathrm{erg} \mathrm{s}^{-1} \mathrm{~cm}^{-2}\right)^{0.094}
\end{aligned}
$$

for $M_{P}>150 M_{\oplus}$.

The reason for this inflation is still debated. Guillot \& Showman (2002) showed that the inflated radii could be understood if $\sim 1 \%$ of the stellar flux received by the planet was transferred into the deep atmosphere below the photosphere. The observed relationships between inflated radii and incident flux appear consistent with this idea. However, it is unclear what is the mechanism for transferring energy into the interior. Mechanisms for inflated radii include downward transport of mechanical energy by atmospheric circulation (Showman \& Guillot 2002), enhanced opacities that help to trap heat in the interior (Burrows et al. 2007a), dissipation of thermal tides (Arras \& Socrates 2010), and tidal heating due to a non-zero eccentricity (Jackson et al. 2008; Ibgui, Burrows, \& Spiegel 2010). The Ohmic dissipation model (Batygin \& Stevenson 2010; Perna, Menou, \& Rauscher 2010) uses the interaction of atmospheric winds and the planetary magnetic field to induce electric currents that heats the interior. Rauscher \& Menou (2013) have modelled the process using a 3D model (see Section 4.1.3) and find that ohmic dissipation can explain the radius of HD 209458b for a planetary magnetic field of 3-10 G. However, Rogers \& Showman (2014) used 3D magnetohydrodynamic simulations of the atmosphere of HD 209458b and found Ohmic dissipation rates orders of magntiude too small to explain the inflated radius.

\subsubsection{Temperature structure}

The dayside spectra of hot Jupiters as defined by the Spitzer IRAC colours (Table 3 and Figure 13) have been used to derive information on the atmospheric temperature structure. If temperature decreases with height then the spectrum shows absorption features due to its atmospheric molecules, but a temperature inversion can cause the same features to appear in emission. A constant temperature (isothermal) atmosphere would shown no spectral features.

The presence of a temperature inversion was first suggested in the infrared spectrum of HD 209458b (Knutson et al. 2008; Burrows et al. 2007a) where a bump in the spectrum at 4.5 and $5.8 \mu \mathrm{m}$ can be understood as water vapour in emission. A number of other cases have been suggested based on Spitzer photometry. It has been suggested that inversions result from absorption of starlight by an absorber high in the atmosphere. Suggestions for the absorber include TiO and VO (Hubeny et al. 2003; Fortney et al. 2008) or photochemically produced sulfur compounds (Zahnle et al. 2009). However, observations have so far failed to detect the presence of $\mathrm{TiO}$ or $\mathrm{VO}$ in eclipse or transit spectroscopy in any of these systems.

Knutson, Howard, \& Isaccson (2010) argue that the presence of an inversion correlates with the activity of the host star, with the temperature inversions being found for planets orbiting inactive stars, whereas the non-inverted atmospheres occur in planets orbiting chromospherically active stars.

However, Madhusudhan \& Seager (2010) have investigated the degeneracies between thermal inversions and molecular abudnances, and find it is often possible to fit both inversion and non-inversion models given the limited data points available from Spitzer photometry.

\subsection{Composition}

\subsubsection{Water vapour, carbon monoxide and methane}

Analogy with brown dwarfs of similar temperatures discussed in Section 2 suggests that the most important species in the near-IR spectra should be $\mathrm{H}_{2} \mathrm{O}, \mathrm{CO}$ and $\mathrm{CH}_{4}$. From the discussion in Section 3.3 and Tables 2- 6, it will be apparent that $\mathrm{H}_{2} \mathrm{O}$ and $\mathrm{CO}$ are indeed detected in quite a number of giant exoplanet systems by a variety of different methods. Evidence for these molecules is found in spectroscopy of direct imaged planets (Section 3.3.1), from high resolution cross correlation methods (Section 3.3.2 and Table 2) and from secondary eclipse (Section 3.3.3, Table 4) and transit (Section 3.3.4 and Table 6) spectroscopy.

The data on $\mathrm{CH}_{4}$ is less clear. Although it is reported, for example, in the NICMOS transmission spectrum of HD 189733b (Swain et al. 2008), high resolution cross correlation studies at the same wavelength do not detect it (de Kok et al. 2013), but do detect CO. This suggests a departure from equilibrium chemistry due to vertical mixing as also suggested by Knutson et al. (2012) based on Spitzer phase curves.

The spectra of directly imaged planets shown in Figure 10 also show $\mathrm{CO}$, but at best very weak evidence for $\mathrm{CH}_{4}$. These are all objects that are cool enough to be in the T dwarf range, but actually show spectra more like those of L dwarfs. The lack of $\mathrm{CH}_{4}$ once again indicates non-equilibrium chemistry (Barman et al. 2011a, 2011b; Skemer et al. 2014; Zahnle \& Marley 2014). Departures from equilibrium chemistry are discussed further in Section 4.2.2.

Recently, however, $\mathrm{CH}_{4}$ has been detected photometrically in the very cool ( $~ 600 \mathrm{~K})$ planetary mass companion GJ 504b (Janson et al. 2013) as described in Section 3.3.1

\subsubsection{Carbon dioxide}

Up to a few years ago $\mathrm{CO}_{2}$ was not considered to be an important species for exoplanet and brown dwarf atmospheres as its predicted equilibrium abundance is quite low. Then Swain et al. (2009a) reported an absorption feature at $2.0 \mu \mathrm{m}$ in the NICMOS dayside emission spectrum of HD189733b that they identified as $\mathrm{CO}_{2}$. This is a relatively weak $\mathrm{CO}_{2}$ band. It has never been seen in brown dwarfs, for example, 
whereas the much stronger $\mathrm{CO}_{2}$ band at $4.2 \mu \mathrm{m}$ has been seen (Yamamura, Tsuji, \& Tanabé 2010; Sorahana \& Yamamura 2012).

Fitting the NICMOS feature at $2.0 \mu \mathrm{m}$ in HD189733b as a $\mathrm{CO}_{2}$ band results in $\mathrm{CO}_{2}$ mole fractions $\sim 10^{-3}$ (Madhusudhan \& Seager 2009; Lee, Fletcher, \& Irwin 2012; Line et al. 2012). This is several thousand times higher than the expected chemical equilibrium abundance for solar composition (Moses et al. 2011) or the observed $\mathrm{CO}_{2}$ abundances in brown dwarfs (Tsuji, Yamamura, \& Sorahana 2011). Inclusion of non-equilibrium processes such as photochemistry does not substantially increase $\mathrm{CO}_{2}$ abundances (Zahnle et al. 2009; Moses et al. 2011). However, $\mathrm{CO}_{2}$ abundances are sensitive to elemental composition increasing quadratically with increasing metallicity (Lodders 2002; Zahnle et al. 2009).

The high $\mathrm{CO}_{2}$ abundance is not clearly seen in other observations of HD189733b. In particular the much stronger $\mathrm{CO}_{2}$ bands at $4.2 \mu \mathrm{m}$ and $15 \mu \mathrm{m}$ are not apparent in the Spitzer secondary eclipse data. Fitting separately to the NICMOS and Spitzer data, Madhusudhan \& Seager (2009) found a much lower $\mathrm{CO}_{2}$ abundance from the Spitzer data consistent with equilibrium predictions. If a model is required to fit both the Spitzer and NICMOS data simultaneously, as in the retrieval analysis of Lee et al. (2012) the result is a very high $\mathrm{CO}_{2}$ abundance to fit the NICMOS data, and then a tightly constrained isothermal temperature profile in the upper atmosphere, which hides the strong $4.2 \mu \mathrm{m}$ and $15 \mu \mathrm{m} \mathrm{CO}$ bands that would otherwise be present.

An alternative interpreation is to disregard the NICMOS $2 \mu \mathrm{m}$ feature, the only evidence pointing to a high $\mathrm{CO}_{2}$ abundance in HD 189733b. Gibson et al. (2011) have argued that NICMOS observations are too sensitive to the method of removing systematics to reliably detect molecular species. In that case it is possible to fit the remaining data on the transmission and dayside emission spectra of HD 189733b very well using equilibrium abundances as shown by DobbsDixon \& Agol (2013) who used the solar composition opacities from Sharp \& Burrows (2007).

\subsubsection{C/O ratios}

A high $\mathrm{C} / \mathrm{O}$ ratio was first suggested for the atmosphere of the highly irradiated hot Jupiter WASP-12b (Madhusudhan et al. 2011) and Madhusudhan (2012) has suggested that $\mathrm{C} / \mathrm{O}$ ratio may be an important parameter for classifying exoplanet atmospheres. If $\mathrm{C} / \mathrm{O}$ is greater than 1.0 (the solar value is about 0.5 ) the chemistry changes substantially for temperatures above about $1500 \mathrm{~K}$, since almost all the oxygen combines with carbon to form $\mathrm{CO}$, and the abundances of other oxygen bearing species, including $\mathrm{H}_{2} \mathrm{O}$ and TiO/VO are substantially reduced. The excess carbon also results in increased abundances of carbon species such as HCN and $\mathrm{C}_{2} \mathrm{H}_{2}$.

Reanalysis of the secondary eclipse data on WASP-12b by Crossfield et al. (2012b), with corrections for the effects of a contaminating star, concluded that the spectrum was wellapproximated by a blackbody and that no constraints on its atmospheric abundances could be set. Other recent studies of the emission and transmission spectra of WASP-12b (Sing et al. 2013; Swain et al. 2013; Mandell et al. 2013) do not clearly detect any molecular species and do not significantly constrain the $\mathrm{C} / \mathrm{O}$ ratio.

Line et al. (2013b) have investigated the ability to determine $\mathrm{C} / \mathrm{O}$ ratios using retrieval models (see Section 4.1.2) and find that with limited data this is very difficult and the retrieved values are biased towards the solar value or a value of one.

\subsection{Clouds and hazes}

The best evidence for cloud or haze ${ }^{3}$ in giant exoplanets comes from observations of the resolved planets (or planetary mass objects) HR 8799b, HR 8799c and 2M 1207b where photometry and spectroscopy point to cloudy atmospheres similar to those of L dwarfs as already discussed in Section 3.3.1. A recent analysis by Skemer et al. (2014) including mid-infrared data concluded that patchy clouds as well as non-equilibrium chemistry (to explain the weakness of the $3.3 \mu \mathrm{m} \mathrm{CH}_{4}$ band) were needed to fit the data for the HR 8799 planets, whereas a thick cloud model fitted the $2 \mathrm{M}$ $1207 \mathrm{~b}$ data.

HD 189733b has good evidence for a Rayleigh scattering haze that is visible in both the transmission spectrum observed during transit (Pont et al. 2013) and in the reflection spectrum from secondary eclipse (Evans et al. 2013). Rayleigh scattering (seen as an increase in radius to the blue) is also seen in the transmission spectrum of WASP-12b (Sing et al. 2013) and WASP-6b (Jordán et al. 2013).

Demory et al. (2013) use an analysis of the optical phase curve and secondary eclipse of Kepler- $7 b$ to conclude that clouds must be present and must have an inhomogenous distribution to explain the lack of symmetry in the phase curve.

The presence of clouds or hazes are suggested in some other systems by essentially featureless transmission spectra that lack features expected for a clear atmosphere such as $\mathrm{Na}$ or $\mathrm{H}_{2} \mathrm{O}$ absorption (e.g. Line et al. 2013a; Gibson et al. 2013a).

\section{ATMOSPHERIC MODELS}

\subsection{Types of models}

Exoplanet and brown dwarf atmospheres occupy a temperature range extending from that of the Solar system planets to that of the coolest stars. Modelling techniques for these objects can thus adapt techniques both from traditional stellar atmosphere modelling (e.g. Gray 2005) and those developed

\footnotetext{
${ }^{3}$ In using the terms 'cloud' or 'haze' here I have followed the terminology used in the orignal reports. I am not aware of any accepted definition of the difference between these two terms, and in this context they likely refer to the same types of particles but haze is usually thinner than cloud and often occurs at higher altitude.
} 
for modelling of the Earth and other Solar system planet atmospheres (e.g. Liou 2002). These two fields have developed largely independently and have significant difference in approach that are now becoming apparent, as methods from both fields are applied to the modelling of exoplanet atmospheres.

Howvever, the essentials of atmospheric modelling are the same for all such objects. The VSTAR modeeling code (Bailey \& Kedziora-Chudczer 2012), for example, has been used successfully for objects ranging from terrestrial (Bailey 2009; Cotton et al. 2012) and giant planets (KedzioraChudczer \& Bailey 2011) in the Solar system to exoplanets (Zhou et al. 2013, 2014), brown dwarfs and cool stars (Bailey \& Kedziora-Chudczer 2012).

\subsubsection{Stellar atmosphere type models}

The traditional approach to stellar atmosphere modelling is typified by the ATLAS series of modelling codes (Kurucz 1970, 1993; Castelli \& Kurucz 2004), and the MARCS models (Gustaffson et al. 2008). Normally with such models the starting point is an adopted effective temperature $T_{\text {eff }}$, surface gravity (usually specified as $\log g$ in cgs units), and metallicity $[\mathrm{M} / \mathrm{H}]$. Grids of models can then be calculated for different values of these parameters. The essential stages in such models are:

1. Start with an initial estimate for the pressure temperature structure of the atmosphere specified at a number ( $\sim 40-80)$ of layers.

2. For each layer calculate the composition of the layer. For hotter stars this primarily involves determining the distribution of ionisation states for each element using Saha's equation. For cooler stars some molecules become important and their concentrations are calculated assuming chemical equilibrium.

3. Calculate the opacity (extinction coefficient) of each layer at each required wavelength taking account of atomic absorption lines, molecular absorption lines and continuum opacity sources such as bound-free and free-free absorption, collision induced absorptions, Rayleigh and electron scattering. The wavelength range must cover all wavelengths at which significant energy transport occurs.

4. Solve the radiative transfer equation to determine the radiative energy flux through each layer.

5. Iteratively adjust the temperature structure of the model, repeating steps $2-4$ as required until the model is in energy balance. The total flux through each layer, including convective energy flux which is normally determined using mixing length theory (Henyey, Vardya, $\&$ Bodenheimer 1965) must equal $\sigma T_{\mathrm{eff}}^{4}$.

The spectrum of the star can then be obtained, either from the last iteration of the model if opacities and radiative transfer are calculated with sufficient resolution, or from a separate spectral synthesis model.
Models based on essentially this procedure have been developed for brown dwarf and exoplanet atmospheres (e.g. Tsuji et al. 1996; Allard et al. 2001; Barman, Hauschildt, \& Allard 2001; Marley et al. 2002; Burrows, Sudarsky, \& Hubeny 2003). In order to model the atmospheres of these cooler objects a number of additional complications have to be dealt with.

At lower temperatures the composition becomes dominated by molecules (as shown in Figure 3), and the calculation of composition (or equation of state) becomes primarily a chemical model. Large numbers of chemical compounds are potentially important and hence large chemical models handling hundreds or in some case thousands of species have been developed (e.g Lodders \& Fegley 2002). (see Section 4.2).

As well as gas phase species, at temperatures below about $2000 \mathrm{~K}$ condensates start to form and both modify the gas phase chemistry, and can form clouds that contribute substantially to the opacity. As we have already seen clouds are important in understanding the behaviour of $\mathrm{L}$ dwarfs and the L/T transition, and are also probably important in giant exoplanets. (see Section 4.4).

Molecules and cloud particles contribute to scattering of light. Scattering is usually a relatively minor contribution to the opacity of stellar atmospheres and is usually treated using simplifying approximations such as that of isotropic scattering. In the cooler atmospheres of brown dwarfs and exoplanets scattering becomes more significant, and more rigorous treatments of scattering that accurately account for the non-isotropic phase functions may be needed (de Kok et al. 2011; Bailey \& Kedziora-Chudczer 2012). (see Section 4.5).

\subsubsection{Retrieval models}

A different approach to modelling exoplanet atmospheres is shown in a number of recent studies (Madhusudhan \& Seager 2009; Line et al. 2012; Lee et al. 2012; Benneke \& Seager 2012; Line et al. 2013b; Benneke \& Seager 2013) that adopt a retrieval approach. These approaches are similar to that used in remote sensing studies of the Earth atmosphere where temperature structure (e.g. Rozenkranz 2001) trace gas content (e.g. Buchwitz et al. 2005), and cloud properties (e.g. Garnier et al. 2012) are routinely retrieved from satellite observations, and similar techniques are used to study the atmospheres of other Solar system planets from orbiting spacecraft or Earth-based telescopes.

These models seek to retrieve the temperature structure and composition of the atmosphere directly from observations, rather than predict these using energy balance and chemical models as in the approach described above. Thus only steps 3 and 4 of the modelling procedure are needed in the forward model. A number of different approaches to the retrieval process have been used. Madhusudhan \& Seager (2009) search in a large grid of models covering a wide parameter space. Lee et al. (2012) use an iterative optimal estimation procedure. Line et al. (2013b) investigate several 
different approaches to retrieval (Optimal Estimation, Bootstrap Monte Carlo, and Differential Evolution Markov Chain Monte Carlo).

Benneke \& Seager $(2012,2013)$ use a somewhat different approach where the temperature profile is not retrieved, but is determined by a self consistent model requiring radiative and hydrostatic equilibrium and allowing for convection. The model can therefore be described by a small number of parameters (planet-to-star radius ratio, cloud-top pressure and Bond albedo) as well as the mole fractions of molecular species. The models include cloud and haze layers and retrieval uses a Bayesian Nested Sampling Monte Carlo method.

\subsubsection{D models of hot Jupiters}

All the models considered so far are 1D models that describe the structure of the atmosphere with a single one dimensional profile. Such models cannot represent dynamical effects and diurnal variations. For hot Jupiters which all receive strong irradiation from their star, the structure is expected to vary substantially around the planet and can be very different on the dayside and nightside.

A number of studies have looked at full 3D models of the atmospheric circulation (General Circulation Models or GCMs) for hot Jupiters. A GCM typically consists of a dynamical core (usually adpated from an Earth atmosphere model) that numerically solves the equations that govern atmospheric circulation over a three dimensional grid of points. These equations can either be the 'primitive equations' that include the approximations of vertical hydrostatic equilibrium and a shallow atmosphere as used by Showman et al. (2009) and Rauscher \& Menou (2012) or the full equations that avoid these approximations as used by Dobbs-Dixon \& Lin (2008) and Mayne et al (2014). Initially simplified schemes were used to represent the forcing from the illuminating star (e.g. Showman \& Guillot 2002; Cooper \& Showman 2005; Menou \& Rauscher 2009) but more recent models include a radiative transfer model and are therfore coupled radiative-dynamical models.

Because of the need to perform radiative transfer solutions for each grid point and time step, radiative transfer methods for GCMs generally need to be simplified compared with those used in the 1D models described earlier. Dobbs-Dixon $\&$ Lin (2008) used a grey model described by a single mean opacity. Heng, Frierson, \& Phillips (2011) and Rauscher \& Menou (2012) use dual-band radiative transfer, dividing radiation into incoming shortwave radiation from the star, and outgoing longwave radiation from the planet. Showman et al. (2009) use the correlated-k method (see Section 4.3) with 30 wavelength bins. Dobbs-Dixon \& Agol (2013) use a similar set of 30 bins but use band-averaged opacities rather than the correlated-k method. The radiative transfer in all these cases uses a two-stream approximation. Amundsen et al. (2014) has tested the accuracy of some of these approaches and find that correlated-k and two-stream methods give reasonable accuracy, but band-averaged opacities can lead to substantial errors.

A review of atmospheric circulation models for exoplanets is given by Showman, Cho, \& Menou (2010). Features predicted by such models are the presence of a superrotating jet in the equatorial regions with wind velocities of 1-4 $\mathrm{km} / \mathrm{s}$. This can cause an eastward displacement of the hottest region from the substellar point which is consistent with observations of HD 189733b (Knutson et al. 2007a, 2009b).

Models that specifically aim to simulate the atmospheres of HD 189733 b and HD 209458b have been given by Showman et al. (2009) and an example of the predicted temperature structure and winds is given in Figure 16 and show reasonable agreement with observed day-night phase variations in Spitzer photometry of HD 189733b (Knutson et al. 2007a, 2009b).

Dobbs-Dixon \& Agol (2013) have used a 3D model of HD $189733 \mathrm{~b}$ including wavelength dependent radiative transfer to make predictions of the transmission spectrum, dayside spectrum, and phase curves that are in good agreement with the observations.

\subsubsection{D models of brown dwarfs}

Studies of atmospheric circulation in brown dwarfs have been made using 3D models and analytic theory (Showman \& Kaspi 2013) and shallow water models (Zhang \& Showman 2014). These show atmospheric circulation with horizontal wind speeds up to $300 \mathrm{~m} \mathrm{~s}^{-1}$, and vertical mixing that could help to explain the disequilibrium chemistry and patchy clouds near the L/T transition (see Section 2.5).

\subsection{Atmospheric chemistry}

\subsubsection{Equilibrim chemistry}

Chemical models for brown dwarf and exoplanet atmospheres aim to predict the chemical composition in the atmosphere given the pressure, temperature and elemental abundances. Normally this is based on the assumption of chemical equilibrium. This can be achieved by solving a system of equations for the mass balance of each element and for the overall charge balance using the equilibrium constants of formation for each compound (e.g. Tsuji 1973; Allard et al. 2001; Lodders \& Fegley 2002). An alternative, but equivalent, approach is that of minimisation of the total Gibbs free energy of the system (Sharp \& Huebner 1990; Sharp \& Burrows 2007).

In either case the required data is available in compilations such as the National Institute for Standards and Technology (NIST)-JANAF thermochemical Tables (Chase 1998) and similar Tables such as Barin (1995) and Robie \& Hemingway (1995). These tables list the equilibrium constants of formation $K_{f}$ and Gibbs free energy of formation $\Delta_{f} G^{o}$ for a large number of compounds as a function of temperature. The two are related through

$$
\Delta_{f} G^{o}=-R T \ln K_{f}
$$




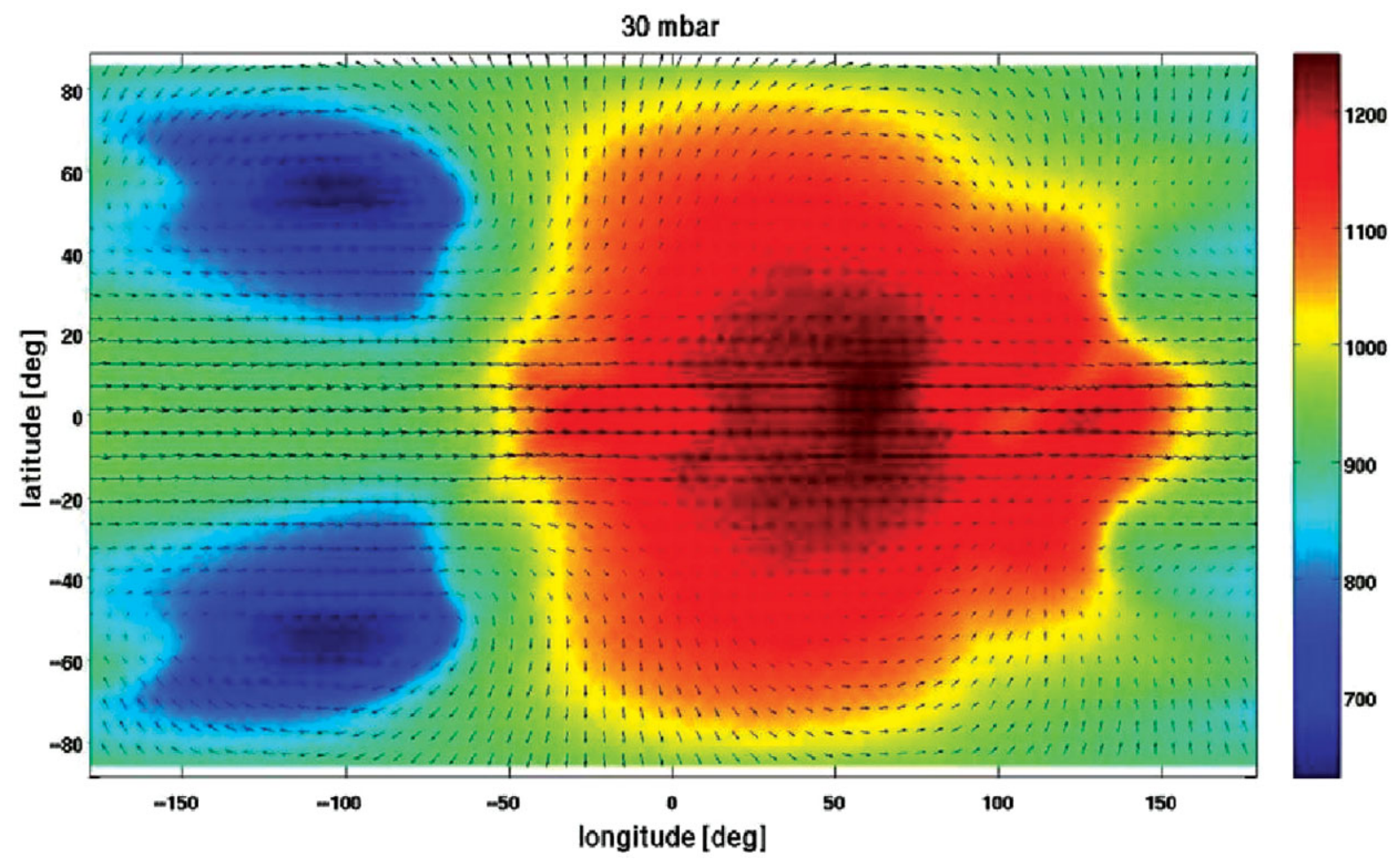

Figure 16. Temperature (colourscale in $\mathrm{K}$ ) and winds (arrows) at the $30 \mathrm{mbar}$ level for a $3 \mathrm{D}$ general circulation model simulation of the atmosphere of HD 189733b. (Figure 4, Showman, A.P. et al., Atmospheric Circulation of Hot Jupiters: Coupled Radiative-Dynamical General Circulation Model Simulations of HD 189733b and HD 209458b, The Astrophysical Journal, 699, 564. reproduced by permission of the AAS.)

Where $\mathrm{R}$ is the gas constant. The required thermochemical data for gas phase species can also be derived from spectroscopic constants.

Chemical models predict the abundances of gas phase species, ionised species and the formation of liquid and solid condensates. The thermochemical models can also predict quantities such as the mean molecular weight, the specific heat and the adiabatic gradient, the latter two quantities being needed for mixing length convection theory.

\subsubsection{Departures from equilibrium}

Departures from equlibrium chemistry can occur as a result of photochemistry or vertical mixing if these processes occur at a faster rate than the collisional processes that tend to restore equilibrium. A non-equilibrium correction to the equilibrium abundances of $\mathrm{CH}_{4} / \mathrm{CO}$ and $\mathrm{NH}_{3} / \mathrm{N}_{2}$ due to vertical mixing (Saumon et al. 2003) has been adopted to explain the observations of these species in brown dwarfs. A similar nonequilibrium treatement is used by Barman et al. (2011a) to model the exoplanet HR8799b. Cooper \& Showman (2006) have found that similar departures from $\mathrm{CO} / \mathrm{CH}_{4}$ equilibrium occur in tidally-locked hot-Jupiters.

Zahnle \& Marley (2014) have explored the disequilibrium abundances of $\mathrm{CH}_{4} / \mathrm{CO}$ and $\mathrm{NH}_{3} / \mathrm{N}_{2}$ in brown dwarfs and self-luminous giant planets using a chemical kinetic approach. They find that the low gravity of planets strongly discriminates against $\mathrm{CH}_{4}$, and that in Jupiter mass planets
$\mathrm{CH}_{4}$ becomes more abundant than $\mathrm{CO}$ only for temperatures below about $400-600 \mathrm{~K}$ depending on the effects of vertical mixing. Ammonia is also sensitive to gravity but insensitive to mixing making it a potetnial proxy for gravity.

Chemical models for hot Jupiter atmospheres using a chemical kinetic approach that can include the effects of photochemistry have been explored in a number of studies (e.g. Zahnle et al. 2009; Line, Liang, \& Yung 2010; Line et al. 2011; Moses et al. 2011; Venot et al. 2012; Agúndez et al. 2014) (see also review by Moses 2014). There remain some differences between model predictions for species such as $\mathrm{CH}_{4}$ and $\mathrm{NH}_{3}$ due to uncertainties in reaction rates and transport parameters (see discussion in Moses 2014 and Agúndez et al. 2014), but generally these models show enhancements of a number of species in the upper atmosphere due to photochemical effects. Most of these models consider $\mathrm{C}, \mathrm{N}$ and O containing species. The model by Zahnle et al. (2009) includes sulfur species and explores the photochemical production of HS (mercapto) and $\mathrm{S}_{2}$ as possible absorbers that could contribute to stratospheric heating.

\subsection{Spectral line absorption}

Absorption lines due to rovibrational and electronic transitions of molecules are the most important features of the spectra of brown dwarfs and planets. Species that are important include $\mathrm{H}_{2} \mathrm{O}, \mathrm{CO}, \mathrm{CH}_{4}, \mathrm{CO}_{2}$ and $\mathrm{NH}_{3}$, metal oxides such 
as $\mathrm{TiO}$ and $\mathrm{VO}$, metal hydrides including $\mathrm{FeH}, \mathrm{CrH}, \mathrm{MgH}$, $\mathrm{CaH}$ and $\mathrm{TiH}$, and carbon species such as $\mathrm{CH}, \mathrm{CN}, \mathrm{C}_{2}, \mathrm{HCN}$ and $\mathrm{C}_{2} \mathrm{H}_{2}$ (particularly in carbon rich atmospheres).

Large numbers of vibrational and rotational levels can be excited at the temperatures of a few thousand degrees encountered in ultracool dwarfs and hot Jupiters. This leads to a requirement for large line lists containing many millions of lines such as the BT2 (Barber et al. 2006) computed line list for $\mathrm{H}_{2} \mathrm{O}$.

The spectral line data are used in models to calculate the absorption in each atmospheric layer. This can be done using on-the-fly line-by-line calculations (Allard et al. 2001; Bailey \& Kedziora-Chudczer 2012) which has the advantage of being the most accurate and flexible method and resulting in high-resolution model spectra. However it is also the most computationally intensive approach.

A faster approach is to precalcuate opacity tables (Sharp \& Burrows 2007; Freedman, Marley, \& Lodders 2008) that are then interpolated for the actual models. However, this can lead to inaccuracies if the wavelength bins are made too large. A widely used appoach in Earth atmosphere modelling is the correlated-k (or k-distribution) method (Goody et al. 1989), which allows the use of larger wavelength bins while retaining accuracy. Recently correlated-k techniques have been used in exoplanet retrieval models (Lee, Fletcher, \& Irwin 2012) and in hot Jupiter GCMs (Showman et al. 2009).

Sources of spectral line data for the important species have been discussed in detail in a number of recent papers (Sharp \& Burrows 2007; Freedman, Marley, \& Lodders 2008; Bailey \& Kedziora-Chudczer 2012; Tennyson \& Yurchenko 2012). These also discuss related continuum absorption processes and the handling of line shapes. The reader is referred to these papers for detailed information, and the discussion here relates only to recent developments.

\subsubsection{Carbon dioxide $\left(\mathrm{CO}_{2}\right)$}

The Carbon Dioxide Spectroscopic Databank (CDSD Tashkun et al. 2003) previously available in $296 \mathrm{~K}$ and 1000 $\mathrm{K}$ versions is now available in a $4000 \mathrm{~K}$ version ${ }^{4}$ contain- $^{-}$ ing lines to an intensity of $10^{-27} \mathrm{~cm}$ molecule ${ }^{-1}$ at $4000 \mathrm{~K}$ for four isotopologues over the range $226-8310 \mathrm{~cm}^{-1}(628$ million lines).

New computed line lists for $\mathrm{CO}_{2}$ and its isotopologues at 296K and $1000 \mathrm{~K}$ (the Ames-296K and Ames-1000K lists) have been described by Huang et al. (2013, 2014).

\subsubsection{Ammonia $\left(\mathrm{NH}_{3}\right)$}

A computed line list for ammonia at temperatures up to 1500 $\mathrm{K}$ and containing more than 1.1 billion lines for frequencies up to $12000 \mathrm{~cm}^{-1}$ (the BYTe list) is described by Yurchenko, Barber, \& Tennyson (2012).
Hargreaves, Li, \& Bernath (2011, 2012a) have provided line lists based on laboratory measurements of $\mathrm{NH}_{3}$ lines at temperatures from $300{ }^{\circ} \mathrm{C}$ to $1400{ }^{\circ} \mathrm{C}$ over the wavelength range from $740-4000 \mathrm{~cm}^{-1}$.

\subsubsection{Methane $\left(\mathrm{CH}_{4}\right)$}

Methane has been the most problematic of the important species in exoplanet and brown dwarf atmospheres as far as line data is concerned. Significant recent progress has been made with modelling (e.g. Rey, Nikitin, \& Tyuterev 2013; Nikitin, Rey, \& Tyuterev 2013b; Yurchenko et al. 2013) and a large computed line list for hot methane has very recently been developed (Yurchenko \& Tennyson 2014) ${ }^{5}$. Yurchenko et al. (2014) have shown that using this line list it is possible to obtain good model fits to the methane bands in the near infrared spectra of brown dwarfs that could not be fitted with older line lists, such as those based on the Spherical Top Data System software (STDS Wenger \& Champion 1998). Another computed line list for hot methane has been reported by Rey, Nikitin, \& Tyuterev (2014) but is limited to wavelengths longer than $2 \mu \mathrm{m}$.

Much improved line lists for the $1.26-1.71 \mu \mathrm{m}$ region at temperatures from $80-300 \mathrm{~K}$ have been developed recently from extensive laboratory measurements at cryogenic and room temperature (Wang et al. 2012; Campargue et al. 2012a, 2013). These lists, and earlier versions of them, have been used successfully for modelling the spectra of Titan (Bailey, Ahlsved, \& Meadows 2011; de Bergh et al. 2012; Campargue et al. 2012b) and Uranus (Irwin et al. 2012; Bott, KedzioraChudczer, \& Bailey 2013). An improved low temperature line list has also been developed for the $2 \mu \mathrm{m}$ region (Daumont et al. 2013). These lists have been incorporated into the new 2012 edition of the HITRAN database ${ }^{6}$ recently released.

Empirical line lists for methane measured at temperatures from 300-1 $400{ }^{\circ} \mathrm{C}$ over the wavelength range from 2.0$10.4 \mu \mathrm{m}$ have been provided by Hargreaves et al. (2012b).

\subsubsection{SiO and $\mathrm{HCN} / \mathrm{HNC}$}

New line lists for SiO (Barton, Yurchenko, \& Tennyson 2013) and HCN/HNC (Barber et al. 2014) have recently been published by the ExoMol group.

\subsubsection{Collision induced absorptions (CIA)}

The collision induced absorption of $\mathrm{H}_{2}-\mathrm{H}_{2}$ and $\mathrm{H}_{2}-\mathrm{He}$ pairs are important contributors to the opacity of brown dwarfs and planets. Updated data on these absorptions have recently been provided by Abel et al. $(2011,2012)$ as described in Saumon et al. (2012). This data as well as other CIA datasets have been recently made available in a new section of the HITRAN database (Richard et al. 2012).

\footnotetext{
5 available at http://www.exomol.com/data/molecules/CH4/12C-1H4

${ }^{6}$ www.cfa.harvard.edu/hitran
}

${ }^{4}$ at ftp://ftp.iao.ru/pub

PASA, 31, e043 (2014)

doi:10.1017/pasa.2014.38 


\subsection{Condensates and clouds}

Condensed phases (i.e. solids and liquids) begin to condense out of the gas when temperatures drop to around $2000 \mathrm{~K}$ and lower. These condensates can form clouds that can significantly alter the opacity and hence the structure of the atmosphere. Chemical models (Section 4.2) can predict which species will condense (these include oxides, silicates and iron) and the amounts of condensed material produced. However, it is harder to predict what size particles will be produced and whether they will remain in place as clouds or fall under gravitation (precipitation, sedimentation or rain-out).

Lorenz-Mie scattering theory can be used to predict the optical properties of the cloud particles. In the general case these include the extinction coefficient, the single scattering albedo (the fraction of light that is scattered rather than absorbed) and the phase function that describes the angular distribution of scattered light. These are needed as inputs for solving the radiative transfer equation (Equations (4) and (5), Section 4.5).

\subsubsection{Clouds in brown dwarfs}

Two limiting cases were considered in the COND and DUSTY models of Allard et al. (2001). The COND models include condensate formation, which alters the chemistry by depleting elements from the gas, but did not include any contribution of the condensates to the opacity. In the DUSTY models the condensed material is assumed to remain in place in equilibrium with the gas phase and form clouds of small dust grains. The DUSTY models were found to be a good representation of late-M and early-L dwarfs, but at cooler temperatures they produce weakening of spectral features and increasingly red colours in disagreement with the observations of $\mathrm{L}-\mathrm{T}$ transition objects.

The cloud-free COND models were found to be a fairly good representation of mid to late $\mathrm{T}$ dwarfs, indicating that gravitational settling has largely removed dust from the atmospheres in these cases. However, neither of these two models could account for the late-L to early $\mathrm{T}$ dwarfs. A number of cloud models have now been developed that aim to reproduce the behaviour of clouds through the full brown dwarf spectral sequence.

In the Unified Cloudy Model (Tsuji 2002, 2005), clouds are assumed to be restricted to a small range of temperatures between the condensation temperature $T_{\text {cond }}$ and a critical temperature $T_{c r}$. Below the critical temperature it is assumed that grains will grow to such a size that they will rapidly precipitate under gravity. A fixed particle size $(r=0.01 \mu \mathrm{m})$ is used in the clouds. The critical temperature $T_{c r}$ is an adjustable parameter, with values in the range $1700-1900 \mathrm{~K}$ providing a reasonable match to the observations.

Burrows, Sudarsky, \& Hubeny (2006) describe a cloud model that similarly restricts the cloud extent but includes an exponential decay in cloud particle density at the upper and lower edges of the cloud. They investigate the effects of various cloud parameters and conclude that cloud particle sizes of $50-100 \mu \mathrm{m}$ fit the data best. This is much larger than the grain sizes used in most other models which are around $1 \mu \mathrm{m}$ or smaller.

Ackerman \& Marley (2001) describe a cloud model based on a balance between turbulent diffusion and sedimentation in horizontally uniform cloud decks. The model involves a scaling factor $f_{\text {sed }}$ that describes the efficiency of sedimentation and typically ranges from 1 to 5 . Small $f_{\text {sed }}$ values produce thicker clouds and match observations of L dwarfs and higher values are found for later type T dwarfs (Stephens et al. 2009).

The BT-Settl models (Allard et al. 2007, 2012) use a cloud treatment based on a model for cloud microphysics from analysis of solar system atmospheres (Rossow 1978) that predicts timescales for condensation, sedimentation and coagulation. These are compared with the turbulent mixing timescale to predict grain densities and sizes.

Woitke \& Helling (2003, 2004) and Helling \& Woitke (2006) have developed a kinetic (non-equilibrium) model for the nucleation, accretion, gravitational settling and evaporation of dust grains. A version of this cloud model has been integrated with the PHOENIX stellar atmosphere code (Hauschildt \& Baron 1999) to provide the DRIFT-PHOENIX models for substellar atmospheres (Helling et al. 2008a).

A more detailed description of some of these different cloud models and a comparison of their predictions in test cases can be found in Helling et al. (2008b).

A specific aim of these models is to explain the changes that occur in brown dwarfs at the $\mathrm{L} / \mathrm{T}$ transition as discussed in Section 2. Figure 17 shows that the BT-Settl model (and other cloud models make simiar predictions) can explain the general trend seen in the near-IR colour magnitude diagram of a swing from red to blue colours at the L/T transition. The models achieve this mostly because the cloud has a limited extent in temperature, and so for cooler models the clouds drop to layers below the photosphere where the effect on the spectra and colours become small.

However, all current models fail to match the details of the L/T transition. As can be seen in Figure 7 models fail to reproduce the sharpness of the transition as a function of effective temperature. Models also fail to reproduce the J-band brightening (see Section 2.3). The BT-Settl model also predicts $\mathrm{J}-\mathrm{K}$ colours that continue to get bluer with lower effective temperatures, while observations show fairly constant $\mathrm{J}-\mathrm{K}$ for mid to late $\mathrm{T}$ dwarfs (Figures 7 and 17).

Cloud species that condense at lower temperatures (including $\mathrm{Cr}, \mathrm{MnS}, \mathrm{Na}_{2} \mathrm{~S}, \mathrm{ZnS}$ and $\mathrm{KCl}$ ) are considered by Morley et al. (2012), and found to be helpful in explaining the colours and spectra of late-T and Y dwarfs (Leggett et al. 2013).

\subsection{Radiative transfer}

Atmospheric models can differ significantly in their handling of radiative transfer, particularly in regards to the treatment of scattering. Radiative transfer involves the flow of radiation 


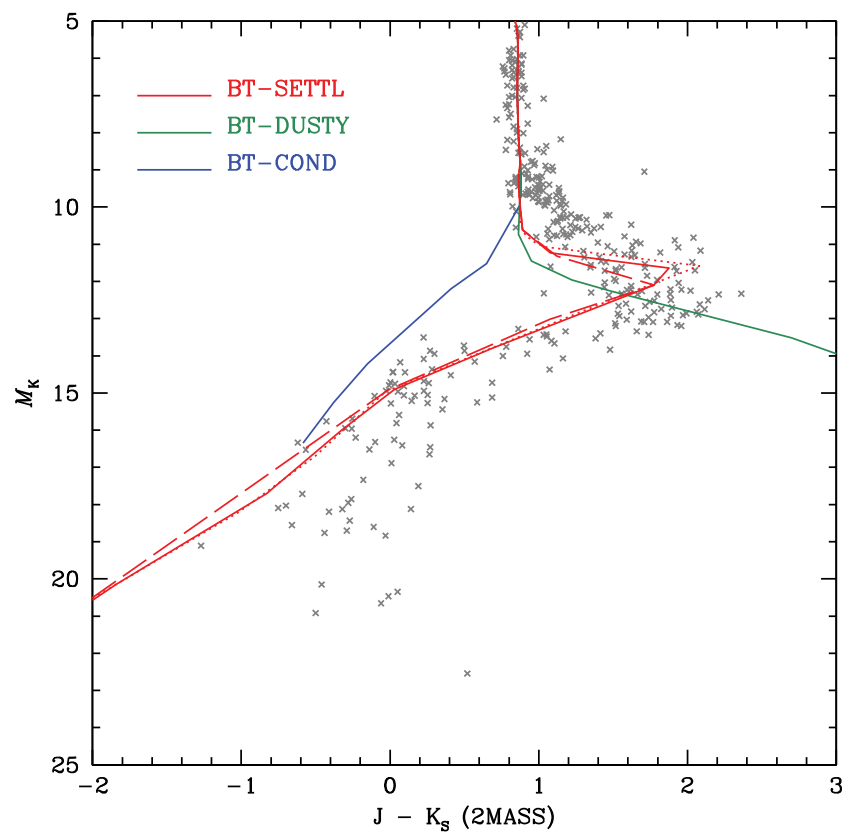

Figure 17. Colour magintude diagram using the same data as Figure 5 compared with the predictions of model atmospheres using different cloud models. The BT-COND and BT-DUSTY models are updated version of the COND and DUSTY models of Allard et al. (2001) with more modern opacities. The BT-SETTL model is described by Allard et al. $(2007,2012)$. The plotted lines are predicted synthetic magnitudes for the isochromes of Baraffe et al. (2003) and Chabrier et al. (2000a). For BT-SETTL 1, 3 and 5 Gyr isochrones are plotted as dotted, solid and dashed lines. For COND and DUSTY the $3 \mathrm{Gyr}$ isochrone only is plotted.

through an atmosphere as determined by the processes of absorption, emission and scattering. The radiative transfer equation can be written as (Bailey \& Kedziora-Chudczer 2012):

$$
\mu \frac{d I_{v}(\tau, \mu, \phi)}{d \tau}=I_{v}(\tau, \mu, \phi)-S_{v}(\tau, \mu, \phi)
$$

where $I_{v}$ is the monochromatic radiance (sometimes referred to as intensity or specific intensity) at frequency $\nu$, and is a function of optical depth $\tau$, and direction $\mu, \phi$, where $\mu$ is the cosine of the zenith angle, and $\phi$ is the azimuthal angle. The source function $S_{v}$ is given by:

$$
\begin{aligned}
S_{v}(\tau, \mu, \phi)= & \frac{\varpi(\tau)}{4 \pi} \int_{0}^{2 \pi} \int_{-1}^{1} P\left(\mu, \phi ; \mu^{\prime}, \phi^{\prime}\right) I_{v}\left(\tau, \mu^{\prime}, \phi^{\prime}\right) d \mu^{\prime} d \phi^{\prime} \\
& +(1-\varpi) B_{v}(T) \\
& +\frac{\varpi F_{v}}{4 \pi} P\left(\mu, \phi ; \mu_{0}, \phi_{0}\right) \exp \left(-\tau / \mu_{0}\right)
\end{aligned}
$$

where the first term describes scattering of radiation into the beam from other directions according to single scattering albedo $\varpi$ and phase function $P\left(\mu, \phi ; \mu^{\prime}, \phi^{\prime}\right)$, the second term is thermal emission, with $B_{v}(T)$ being the Plank function and the third term is direct illumination of the atmosphere by an external source with flux $\mu_{0} F_{v}$ and direction $\mu_{0}, \phi_{0}$ (e.g. the Sun or host star).
It is the first term in Equation (5) involving the double integral that significantly complicates radiative transfer. This term has the consequence that the radiance in any one direction is dependent on the radiance in all other directions (since any of these can potentially scatter into the line of sight). In general it is then only possible to solve for the full angular dependence of the radiation field in all directions.

To avoid this complication the handling of scattering is often simplified, in some cases by ignoring it entirely, or by using a simplified form for the phase function $P$ such as the assumption of isotropic scattering, and/or a simplified form for the angular depedence of $I_{v}$ such as the two-stream approximation or the Eddington approximation. In stellar atmospheres approximate methods can be justified by the fact that scattering is generally of minor importance, and where it does become significant, in the form of Rayleigh scattering from molecules in cool stars, the phase functions are at least forward-backward symmetric.

Where clouds are present, however, the phase functions can be highly non-isotropic, and in the case of Solar system planet atmospheres, radiative transfer methods that more rigorously handle multiple scattering with non-isotropic phase functions are generally used. These include, in particular, versions of the discrete ordinate method originally due to Chandrasekhar (1960) which has been developed into robust and general radiative transfer solving codes such as DISORT (Stamnes et al. 1988), SHDOM (Evans 1998) and LIDORT (Spurr 2001). DISORT is used by Bailey \& KedzioraChudczer (2012) in the VSTAR code to model brown dwarf spectra. Another appropriate method is the adding-doubling method (de Kok et al. 2011).

At present such methods are rarely used in exoplanet and brown dwarf atmospheric modelling, and this opens up the possibility of errors being introduced due to an oversimplified treatment of scattering. This was investgated by de Kok et al. (2011) for the thermal emission spectra of exoplanets who found that substantial errors can be introduced by neglecting scattering, or by using an isotropic scattering approximation where large particles are present.

\subsection{Polarisation}

Scattering processes polarise light, so a full treatment of radiative transfer should take account of polarisation. Light scattered from planetary atmospheres is expected to be polarised whereas the light of normal stars is mostly unpolarised (Bailey, Lucas, \& Hough 2010), and this polarisation can potentially be used as a means of differentially detecting planets in imaging observations (Schmid et al. 2005; Keller 2006), and as a means of characterising extrasolar planet atmospheres by observing the phase variation of polarisation (Seager, Whitney, \& Sasselov 2000; Bailey 2007). Polarisation has also been measured in some brown dwarfs (Ménard, Delfosse, \& Monin 2002; Zapatero Osorio, Caballero, \& Béjar 2005; Tata et al. 2009) and is thought to result from scattering in the dust clouds combined with either rotational 
oblateness or a patchy cloud structure (Sengupta \& Marley 2010).

Polarisation is particularly useful for determining the nature and size of cloud particles. A classic application of polarisation was the analysis of the polarisation phase curve of Venus by Hansen \& Hovenier (1974) to determine that the clouds of Venus were composed of $\sim 1 \mu \mathrm{m}$ radius sulfuric acid droplets.

Polarisation should also, ideally, be included in all radiative transfer modelling involving scattering, because even if we are not interested in observing polarisation, neglecting polarisation can alter the resulting fluxes. Stam \& Hovenier (2005) investigated this for reflected light from extrasolar giant planets and found that errors in geometric albedo of up to $9 \%$ could arise as a result of neglecting polarisation. In practice, however, polarisation is normally ignored in radiative transfer calculations, because including polarisation would substantially slow down the computations.

Polarisation in Earth-like planet atmospheres will be discussed later in Section 5.4.

\section{THE SEARCH FOR HABITABLE PLANETS AND LIFE}

The main focus of this review has been on the study of planetary atmospheres for which we have observations, and so far this has been almost entirely giant planets. The only exceptions to this are the super-Earths GJ 1214b and HD 97658b. Transit spectroscopy of GJ 1214b has been obtained (Bean et al. 2010; Crossfield et al. 2011; Berta et al. 2012; Kreidberg et al. 2014a) showing a featureless spectrum indicating an atmosphere either rich in water vapour, or with high altitude clouds. Transit spectroscopy of HD 97658b (Knutson et al. 2014b) also shows a featureless spectrum inconsistent with a cloud-free solar metallicity atmosphere.

In this section the potential for characterisation of Earthlike planets in the habitable zone of their stars is briefly reviewed.

\subsection{The habitable zone}

The habitable zone is defined as the range of distances from its star at which a planet has suitable conditions for liquid water to be able to exist at its surface. In the absence of an atmosphere the average surface temperature $T_{e q}$ of a planet is given by energy balance between radiation received from its star, and thermal radiation to space as:

$$
(1-a) S / 4=\sigma T_{e q}^{4}
$$

Where $S$ is the total incident flux $\left(\mathrm{W} \mathrm{m}^{-2}\right)$ received from the star (in the case of the Earth this is the solar constant $S_{0}=1361 \mathrm{~W} \mathrm{~m}^{-2}$ ), $a$ is the Bond albedo of the planet and $\sigma$ is the Stefan-Boltzmann constant. The factor of 4 comes from the fact that radiation received over an area of $\pi r^{2}$ is redistributed over the entire surface of the planet with area $4 \pi r^{2}$. For Earth this calculation gives an equilibrium temperature of $T_{e q} \sim 255 \mathrm{~K}$ currently, and lower in the past as the solar luminosity increases with time and was about $30 \%$ less early in the Sun's history (Bahcall, Pinsonnealt, \& Basu 2001).

The global average temperature of the Earth is, of course, higher than this at about $T \sim 288 \mathrm{~K}$, with the difference being due to the operation of the greenhouse effect that traps some of the outgoing radiation and causes the outgoing flux to be less than $\sigma T_{e q}^{4}$. In general, from observations of the orbit of a planet we can determine $S$, but in most cases we won't know the albedo $a$ or the amount of the greenhouse effect, and so can't directly determine the surface temperature of a planet from observations. According to Selsis et al. (2007) $T_{e q}$ must be less than $270 \mathrm{~K}$ for a planet to be habitable.

Estimates of the locations of the edges of the habitable zone have been made based on the assumption of an Earth-like planet with a $\mathrm{CO}_{2} / \mathrm{H}_{2} \mathrm{O} / \mathrm{N}_{2}$ atmosphere using cloud-free $1 \mathrm{D}$ radiative-convective models. Kasting, Whitmire, \& Reynolds (1993) gave the extent of the habitable zone from 0.95 AU to 1.37 AU for our Solar system, with the inner edge being set by the onset of the moist greenhouse process (Kasting 1988) causing loss of water to space, and the outer edge being set by cooling due to the formation of $\mathrm{CO}_{2}$ clouds. However Forget \& Pierrehumbert (1997) showed that $\mathrm{CO}_{2}$ clouds actually cause warming and allow a more extended habitable zone. An upadated calculation is given by Kopparapu et al. (2013) which sets the moist greenhouse inner edge at $0.99 \mathrm{AU}$, and the outer edge at $1.67 \mathrm{AU}$ based on the maximum greenhouse criterion. On this basis the Earth is near the inner edge of the habitable zone.

These results can be scaled for other stars according to $S / S_{0}$, the flux received by the star as a fraction of the solar constant and the effective temperature of the star. A habitable zone calculator for this puropse based on the results of Kopparapu et al. (2013) is available ${ }^{7}$. The effects of different planet masses on the position of the habitable zone are considered by Kopparapu et al. (2014) who find that the inner edge moves so as to give a wider zone for higher mass planets.

Recent studies using 3D climate models, however, have found the inner edge of the habitable zone at $\sim 0.95 \mathrm{AU}$ (Leconte et al. 2013) or $\sim 0.93$ AU (Wold \& Toon 2014), significantly smaller than the 1D models described above.

The results assume an Earth-like planet and could be different for other types of planets. Abe et al. (2011) have shown that a more extended habitable zone is possible for a desert planet with limited surface water, and Zsom et al. (2013) find a miminum inner edge for the habitable zone of $0.38 \mathrm{AU}$ for hot desert worlds. Pierrehumbert \& Gaidos (2011) have suggested that the greenhouse effect due to collision induced absorption in molecular hydrogen could allow habitable conditions to be maintained out to $10 \mathrm{AU}$ from a G-type star.

\footnotetext{
${ }^{7}$ http://depts.washington.edu/naivpl/content/hz-calculator
} 
Table 9. Low mass planets in or near the habitable zone.

\begin{tabular}{|c|c|c|c|c|c|c|c|}
\hline \multirow[b]{2}{*}{ Planet } & \multicolumn{2}{|c|}{ Star } & \multirow[b]{2}{*}{$\begin{array}{c}\mathrm{M} \sin i \\
\text { (days) }\end{array}$} & \multirow[b]{2}{*}{$\begin{array}{c}\text { Radius } \\
\mathbf{M}_{\oplus}\end{array}$} & \multirow[b]{2}{*}{$\mathrm{R}_{\oplus}$} & \multirow[b]{2}{*}{$S / S_{0}$} & \multirow[b]{2}{*}{ Reference } \\
\hline & Type & $\begin{array}{c}\text { Period } \\
\mathrm{T}_{\text {eff }}\end{array}$ & & & & & \\
\hline$\tau$ Cet e & G8.5V & 5344 & 168.1 & 4.3 & & 1.60 & Tuomi et al. (2012) \\
\hline HD $40307 \mathrm{~g}$ & $\mathrm{~K} 2.5 \mathrm{~V}$ & 4956 & 197.8 & 7.1 & & 0.62 & Tuomi et al. (2013) \\
\hline HD $88512 b$ & $\mathrm{~K} 5 \mathrm{~V}$ & 4715 & 58.43 & 3.6 & & 1.86 & Pepe et al. (2011) \\
\hline GJ $163 \mathrm{c}$ & M3.5 & & 25.63 & 6.8 & & 1.34 & Bonfils et al. (2013) \\
\hline GJ $667 \mathrm{C} \mathrm{c}$ & $\mathrm{M} 1.5 \mathrm{~V}$ & 3350 & 28.14 & 3.8 & & 0.90 & Anglada-Escudé et al. $(2012,2013)$ \\
\hline GJ $667 \mathrm{C} \mathrm{e}$ & M1.5V & 3350 & 62.24 & 2.7 & & 0.33 & Anglada-Escudé et al. (2013) \\
\hline GJ 667C f & $\mathrm{M} 1.5 \mathrm{~V}$ & 3350 & 39.03 & 2.7 & & 0.58 & Anglada-Escudé et al. (2013) \\
\hline GJ 832c & M1.5V & 3472 & 35.68 & 5.4 & & 0.87 & Wittenmyer et al. (2014) \\
\hline Kapteyn b & M1.0 & 3570 & 48.616 & 4.8 & & 0.4 & Anglada-Escudé et al. (2014) \\
\hline Kepler-22b & G5V & 5518 & 289.9 & & 2.38 & 1.09 & Borucki et al. (2012) \\
\hline Kepler-61b & M0 & 4017 & 59.88 & & 2.15 & 1.26 & Ballard et al. (2013) \\
\hline Kepler-62e & $\mathrm{K} 2 \mathrm{~V}$ & 4925 & 122.4 & & 1.61 & $1.2 \pm 0.2$ & Borucki et al. (2013) \\
\hline Kepler-62f & $\mathrm{K} 2 \mathrm{~V}$ & 4925 & 267.3 & & 1.41 & $0.41 \pm 0.05$ & Borucki et al. (2013) \\
\hline Kepler-69c & G4V & 5638 & 242.5 & & 1.71 & 1.91 & Barclay et al. (2013); Kane et al. (2013) \\
\hline Kepler-186f & M1V & 3788 & 129.9 & & 1.11 & $0.32_{-0.04}^{+0.06}$ & Quintana et al. (2014) \\
\hline
\end{tabular}

\subsection{Habitable zone planets}

Table 9 lists planets that have been reported as being in or near the habitable zone with $\mathrm{M} \sin i<10 M_{\oplus}$ or $R<2.5 R_{\oplus}$. Note that the planet of $\tau$ Cet is only reported as a tentative detection (Tuomi et al. 2012) and the reality of some of the planets of GJ 667C have been disputed (Gregory 2012; Feroz \& Hobson 2014). The reported habitable zone planets of GJ 581 (Mayor et al. 2009; Vogt et al. 2010) have been excluded from the table based on the analysis of Robertson et al. (2014).

Petigura, Howard, \& Marcy (2013) have analysed Kepler data to find 10 planet candidates with radii of $1-2 \mathrm{R}_{\oplus}$ and within a habitable zone defined by $0.25<S / S_{0}<4$. Allowing for incompleteness they estimate that Earth-size planets in the habitable zone occur in $22 \pm 8 \%$ of stars. With the narrower definition of the habitable zone discussed above (0.99-1.67 AU, Kopparapu et al. 2013) this becomes 8.6\%.

\subsection{Detecting and characterising earth-like planets}

The direct detection and characterisation of Earth-like planets is far more challenging than for the giant planets discussed in Section 3. The contrast ratio between an Earth-like planet and its star is $\sim 10^{-10}$ at visible wavelengths and $\sim 10^{-7}$ in the thermal IR $(\sim 10 \mu \mathrm{m})$.

One concept is that of an infrared interferometer in space as first suggested by Bracewell (1978). This was developed into the Darwin (Cockell et al. 2009) and Terrestrial Planet Finder Interferometer (TPF-I, Beichman, Woolf, \& Lindensmith 1999) mission concepts. These involved several infrared telescopes flying in formation in space and combining their light to achieve nulling interferometry, so that the light of the star could be suppressed, and reveal the light of the planet. These missions would have aimed to both detect plan- ets and obtain low resolution spectroscopy over the 6-20 $\mu \mathrm{m}$ range for atmospheric characterisation and biosignature detection.

An alternative concept was the Terrestrial Planet Finder Coronograph (TPF-C, Traub et al. 2006). This was envisaged as a space telescope with an 8 by $3.5 \mathrm{~m}$ elliptical mirror, using advanced coronographic techniques to suppress starlight at the $10^{-10}$ level. It operated at visible wavelengths and would be able to detect planets and carry out spectroscopic characterisation.

Both Terrestrial Planet Finder missions (TPF-I and TPFC) have now been cancelled by NASA, and the ESA Darwin mission study ended in 2007.

A further concept for starlight suppression involves the use of an occulter (or starshade) placed in front of the telescope. The occulter must use a petal shaped design to suppress diffraction and be placed about $40000 \mathrm{~km}$ in front of the telescope (Cash 2006). An occulter could be used in conjunction with the James Webb Space Telescope (Soummer 2009) and/or with a dedicated space telescope as in the New Worlds Observer (NWO, Turnbull et al. 2012) mission concept. NWO would use a $4 \mathrm{~m}$ telescope and enable detection and spectroscopic characterisation of exoplanets with $\mathrm{R} \sim 100$ over $0.3-1.6 \mu \mathrm{m}$. The mission aims to achieve a $95 \%$ probability of detecting and characterising at least one habitable zone Earth-like planet. A more recent starshade mission concept is the Exo-S mission described in Section 6.

Extreme adaptive optics systems on giant ground-based telescopes are another potential approach to the detection and characterisation of Earth-like exoplanets. However, a number of studies have concluded that such systems on currently planned extremely large telescopes fall well short of the required sensitivity (Stapelfeldt et al. 2005; Mountain et al. 2009). However, (Angel 2003) has suggested that detection 
with a 20-m ground-based telescope and spectroscopic characterisation with a 100-m telescope should be feasible taking advantage of an Antarctic site.

Kaltenegger \& Traub (2009) considered the feasibility of characterising Earth-like planets using transit spectroscopy from a 6.5-m telescope in space (e.g. the JWST). They found that the signal-to-noise values for all important spectral features were of the order of unity or less per transit.

The situation for transit observations is much improved if the Earth-like planet is assumed to be in the habitable zone of an M-dwarf rather than a solar type star. This leads to both a larger transit signal, since the star is smaller, and more frequent transits. Pallé, Zapatero Osorio, \& García Mun'oz (2011) conclude that detection of atmospheric features in transiting Earth-like planets could be possible in planets orbiting very cool stars or brown dwarfs with the proposed 42-m European Extremely Large Telescope.

Snellen et al. (2013) have suggested the use of high resolution cross correlation techniques (see Section 3.3.2) to detect oxygen absorption during the transit of an Earth-like planet across a red dwarf star. They suggest this should be feasible with a ground-based telescope with an effective aperture of $\sim 100 \mathrm{~m}$ and suggest this could be an array of low-cost 'flux collectors' which would not require high image quality.

\subsection{Signatures of habitability}

The presence of a planet within the habitable zone does not necessarily mean that it has habitable conditions on its surface. The best signature of habitability would be direct detection of the presence of liquid water. This is difficult to achieve using spectroscopy. While atmospheric water vapour can be detected through absorptions in the near-IR or thermal infrared, the presence of surface liquid water does not provide any clear spectroscopic signature.

A possible indicator of liquid water would be the presence of the 'glint' signal due to specular reflection from oceans. Williams \& Gaidos (2008) modelled the light curves and polarisation phase depenence for Earth-like planets and showed that distinctive signals due to glint should be detectable for planets with surface oceans. Robinson, Meadows, \& Crisp (2010) used an Earth simulation to show that glint increases the brigthness of the Earth by as much as $100 \%$ at crescent phases. Zugger et al. (2010, 2011) modelled glint effects including polarisation and found that the glint signal becomes diluted and more difficult to detect when clouds, aerosols and surface winds are included. Cowan, Abbott, \& Voigt (2012a) show, however, that latitude dependent albedo variations produce a signal in the phase curve very similar to that from glint, and therefore the glint signal may not be a reliable indicator of the presence of oceans.

Another potential way of detecting the presence of oceans is to use rotational changes in the brightness and colours (Ford, Seager, \& Turner 2001; Kawahara \& Fujii 2010). Such observations can in principle determine the fraction of ocean and land coverage and even provide maps of the distribution. These ideas have been tested using observations of the integrated Earth from the EPOXI mission with the Deep Impact spacecraft (Cowan et al. 2009; Fujii et al. 2011; Cowan \& Strait 2013). Langford, Wyithe, \& Turner (2009) used lunar Earthshine measurements to detect photometric changes associated with the passage of the specular reflection glint spot from land to ocean.

Liquid water clouds in a planet's atmosphere could be detected through the presence of the primary rainbow which would appear as a peak in the phase curve at a phase angle of about 40 degrees. While the rainbow peak could be visible in the phase light curve, it is better defined, particularly for small cloud particles, in the polarisation phase curve (Bailey 2007; Karalidi et al. 2012). The size of the disk integrated rainbow polarisation signal for the Earth has not been measured, but in principle could be obtained from lunar earthshine polarisation measurements. Current data however does not have sufficient phase coverage (Sterzik, Bagnulo, \& Palle 2012; Takahashi et al. 2013; Bazzon, Schmidt, \& Gisler 2013).

\subsection{Biosignatures}

The ultimate aim of such studies is to make observations that would test for the presence of life on an exoplanet. A number of potential biosignatures have been suggested (e.g. Des Marais et al. 2002) and tests have been made to determine whether these are actually visible in observations of the integrated Earth using spacecraft (Livengood et al. 2011) or lunar Earthshine measurements (Woolf et al. 2002). Models of the integrated Earth spectrum have been presented by Tinetti et al. (2006) and Robinson et al. (2011).

The most important biosignature is generally considered to be atmospheric oxygen $\left(\mathrm{O}_{2}\right)$ or ozone $\left(\mathrm{O}_{3}\right)$. Oxygen is produced in the Earth atmosphere primarily by photosynthetic organisms. Possible abiotic sources of atmospheric oxygen that could lead to 'false positivies' have been discussed (Schindler \& Kasting 2000; Selsis, Despois, \& Parisot 2002; Segura et al. 2007) but such cases appear to be unlikely, or can be excluded on the basis of other observations. Atmospheric $\mathrm{O}_{2}$ is detectable through a number of electronic absorption bands with the strongest being the A-band at around $760 \mathrm{~nm}$. The longest wavelength $\mathrm{O}_{2}$ band with significant strength is the a-X band at $1.27 \mu \mathrm{m}$. The lack of $\mathrm{O}_{2}$ bands further into the infrared is a problem for life detection with instruments that work optimally at near-IR wavelengths (e.g. ground-based telescopes with extreme adaptive optics systems that work best at longer wavelenths). However, in the thermal infrared the band of ozone $\left(\mathrm{O}_{3}\right)$ at $9.7 \mu \mathrm{m}$ can be used. This is also considered to be a good biosignature since $\mathrm{O}_{3}$ is a photolytic by-product of $\mathrm{O}_{2}$.

Methane $\left(\mathrm{CH}_{4}\right)$ is another potential biosignature. On Earth it originates largely from biological processes (methanogenic archaea), but there are also possible abiotic sources such as serpentenisation. The simultaneous presence of both oxygen and methane was suggested to be a good biosignature by Lovelock (1965) indicating chemical disequilibrium. 
Methane has strong absorption bands at 7 and $3.3 \mu \mathrm{m}$ and a series of weaker bands through the near-IR. Segura et al. (2005) have suggested that $\mathrm{CH}_{4}$ and other reduced biogenic gases such as $\mathrm{N}_{2} \mathrm{O}$ and $\mathrm{CH}_{3} \mathrm{Cl}$ might be useful as biosignatures in planets around $\mathrm{M}$ dwarfs where these gases would have longer photochemical lifetimes than on Earth.

Another possible biosignature is the 'red edge', the sharp edge in the reflectance spectrum from vegetation at around 700-nm. This is a strong signal in light reflected directly from a vegetated area. However, in the integrated Earth, lunar Earthshine observations show a maximum effect of a few per cent (Hamdani et al. 2006; Arnold 2008). Kiang et al. (2007) suggested that spectral signatures could be different for photosynthetic pigments adapted for different stellar types, and Sanromá et al. (2014) investigate the spectra of the Archaean Earth when purple bacteria were widespread giving rise to a slightly longer wavelength signal.

\section{THE FUTURE}

The characterisation of exoplanet atmospheres has made substantial progress over the last few years and some aspects of their composition and structure are beginning to be resolved. However, there are still many uncertainties and controversies that remain. The major limitation is in the observational data which are, in most cases, extremely limited in spectral resolution and wavelength coverage. Data from space instruments have proved most valuable for studying the transmission and dayside emission spectra of transiting planets, and so the next major advance is likely to be the James Webb Space Telescope due for launch in 2018. Its NIRSpec and MIRI instruments should make possible spectra of transiting planets across the near-IR and mid-IR spectral regions (Shabram et al. 2011; Belu et al. 2011).

Another important mission is TESS (Ricker et al. 2014) that will carry out an all sky survey of bright stars for transiting planets. While it is not directly aimed at atmospheric characterisation, it may well find some of the best targets for more detailed studies. The ESA PLATO mission planned for 2024 launch (Rauer et al. 2013) is another mission that will search for planets transiting bright stars, and will target up to 1000000 stars. An exoplanet characterisation mission EChO (Tinetti et al. 2012) was also proposed for the same launch opportunitiy but was not selected.

NASA is currently studying three missions with relevance to exoplanet characterisation. The Wide-Field Infrared Survey Telescope - Astrophysics Focused Telescope Assets (WFIRST-AFTA) concept (Spergel et al. 2013) is a 2.4-m wide field near infrared $(0.6-2.0 \mu \mathrm{m})$ telescope. It is mainly aimed at wide field surveys, but the design includes a coronograph that enables it do characterisation observations of exoplanets by direct imaging. Exo-C (Stapelfeldt et al. 2014) is a 1.5-m unobscured Cassegrain telescope with a coronograph providing imaging of exoplanets over the wavelength range 450-1 $000 \mathrm{~nm}$. Exo-S (Seager et al. 2014) is a 1.1-m telescope using the starshade concept (see Section 5.3) to provide imaging over the 400-1 $000 \mathrm{~nm}$ range. Exo-S should have the capability to detect Earth-size planets in the habitable zones of about 20 Sun-like stars.

New facilities on ground-based telescopes include improved instruments for direct imaging such as the Gemini Planet Imager (GPI Macintosh et al. 2012) and SPHERE for the VLT (Bezuit et al. 2010). The new generation of extremely large telescopes now under development will open up new possibilities with planned instrument such as EPICS for the $42 \mathrm{~m}$ E-ELT (Kasper et al. 2010) which will provide imaging, spectroscopy and polarimetry with a systematic contrast of $10^{-9}$ at 100 mas separation.

\section{ACKNOWLEDGEMENTS}

This research has benefitted from the M, L, T, and Y dwarf compendium housed at DwarfArchives.org. Use was also made of the $\mathrm{L}$ and $\mathrm{T}$ dwarf data archive of Sandy Leggett, the IRTF spectral library and the Database of Ultracool parallaxes maintained by Trent Dupuy. I thank Daniel Cotton and Brett Addison for valuable comments on the manuscript.

The work is supported by the Australian Research Council through Discovery grant DP110103167.

\section{References}

Abe, Y., Abe-Ouchi, A., Sleep, N. H., \& Zahnle, K. 2011, AsBio, 11,443

Abel, M., Frommhold, L., Li, X., \& Hunt, K. L. C. 2011, JPCA, 115,6805

Abel, M., Frommhold, L., Li, X., \& Hunt, K. L. C. 2012, JChPh, 136, 044319

Ackerman, A. S., \& Marley, M. S. 2001, ApJ, 556, 872

Agol, E., Cowan, N. B., Knutson, H. A., Deming, D., Steffen, J. H., Henry, G. W., \& Charbonneau, D. 2010, ApJ, 721, 1861

Agúndez, M., Parmentier, V., Venot, O., Hersant, F., \& Selsis, F. 2014, A\&A, 564, A73

Albert, L., Artigau, E., Delorme, P., Reylé, C., Forveille, T., Delfosse, X., \& Willott, C. J. 2011, AJ, 141, 203

Allard, F., Hauschildt, P. H., Alexander, D. R., Tamanai, A., \& Schweitzer, A. 2001, ApJ, 556, 357

Allard, N. F., Allard, F., Hauschildt, P. H., Kielkopf, J. F., \& Machin, L. 2003, A\&A, 411, 473

Allard, F., Allard, N. F., Homeier, D., Kielkopf, J., McCaughrean, M. J., \& Spiegelman, F. 2007, A\&A, 474, L21

Allard, F., Homeier, D., Freytag, B., \& Sharp, C. M. 2012, in Low-Mass Stars and the Transition Stars/Brown Dwarfs - Evry Schatzman School on Stellar Physics XXIII, ed. C. Reylé, C. Charbonnel, M. Schultheis, EAS Publications Series, 57, 3

Amundsen, D. S., Baraffe, I., Tremblin, P., Manners, J., Hayek, W., Mayne, N. J., \& Acreman, D. M. 2014, A\&A, 564, A59

Anderson, D. R., et al. 2011, MNRAS, 416, 2108

Anderson, D. R., et al. 2013, MNRAS, 430, 3422

Andrei, A. H., et al. 2011, AJ, 141, 54

Angel, R. 2003, in Proceedings of the Conference on Towards Other Earths: Darwin/TPF and the Search for Extrasolar Terrestrial Planets, ed. M. Fridlund, \& T. Henning, ESA SP-539, 221 (Netherlands: ESA, Noordwijk) 
Angerhausen, D., DeLarme, E., Morse, J. A. 2014, submitted to ApJ, arXiv:1404.4348

Anglada-Escudé, G., et al. 2012, ApJL, 751, L16

Anglada-Escudé, G., et al. 2013, A\&A, 556, 126

Anglada-Escudé, G., et al. 2014, MNRAS, 443, L89

Arnold, L. 2008, SSRv, 135, 323

Arras, P., \& Socrates, A. 2010, ApJ, 714, 1

Artigau, E., Bouchard, S., Doyon, R., \& Lafrenière, D. 2009, ApJ, 701,1534

Bailey, J. 2007, AsBio, 7, 320

Bailey, J. 2009, Icar, 201, 444

Bailey, J., \& Kedziora-Chudczer, L. 2012, MNRAS, 419, 1913

Bailey, J., Lucas, P. W., \& Hough, J. H. 2010, MNRAS, 405, 2570

Bailey, J., Ahlsved, L., \& Meadows, V. S. 2011, Icar, 213, 218

Bahcall, J. N., Pinsonneault, M. H., \& Basu, S. 2001, ApJ, 555, 990

Baines, E. K., et al. 2012, ApJ, 761, 57

Ballard, A., et al. 2013, ApJ, 773, 98

Bakos, G., Noyes, R. W., Kovács, G., Stanek, K. Z., Sasselov, D. D., \& Domsa, I. 2004, PASP, 116, 266

Banfield, D., Gierasch, P. J., Bell, M., Ustinov, E., Ingersoll, A. P., Vasavada, A. R., West, R. A., \& Belton, M. J. S. 1998, Icar, 135, 230

Baraffe, I., Chabrier, G., Allard, F., \& Hauschildt, P. 2002, A\&A, 382,563

Baraffe, I., Chabrier, G., Barman, T. S., Allard, F., \& Hauschildt, P. H. 2003, A\&A, 402, 701

Baraffe, I., Chabrier, G., \& Barman, T. 2008, A\&A, 482, 315

Baraffe, I., Chabrier, G., \& Barman, T. 2010, RPPh, 73, 016901

Barber, R. J., Tennyson, J., Harriss, G. J., \& Tolchenov, R. N. 2006, MNRAS, 368, 1087

Barber, R. J., Strange, J. K., Hill, C., Polyansky, O. L., Mellau, G. Ch., Yurchenko, S. N., \& Tennyson, J. 2014, MNRAS, 437, 1828

Barclay, T., et al. 2013, ApJ, 768, 101

Barin, I. 1995, Thermochemical Data of Pure Substances (3rd edn; Weinheim: VCH-Verlag)

Barman, T. 2007, ApJ, 661, L191

Barman, T. S., Hauschildt, P. H., \& Allard, F. 2001, ApJ, 556, 885

Barman, T. S., Macintosh, B., Konopacky, Q. M., \& Marois, C. 2011a, ApJ, 733, 65

Barman, T. S., Macintosh, B., Konopacky, Q. M., \& Marois, C. 2011b, ApJL, 7335, L39

Barnes, J. R., et al. 2010, MNRAS, 401, 445

Barton, E. J., Yurchenko, S. N., \& Tennyson, J. 2013, MNRAS, 434, 1469

Baskin, N. J., et al. 2013, ApJ, 773, 124

Basri, G., \& Brown, M. E. 2006, Ann. Rev. Earth Planet. Sci., 34, 193

Basri, G., Marcy, G. W., \& Graham, J. R. 1996, ApJ, 458, 600

Batalha, N. M., et al. 2013, ApJS, 204, 24

Batygin, K., \& Stevenson, D. J. 2010, ApJL, 714, L238

Bazzon, A., Schmid, H. M., \& Gisler, D. 2013, A\&A, 556, A117

Bean, J. L., Miller-Ricci Kempton, E., \& Homeier, D. 2010, Nature, 468,669

Bean, J. L., Désert, J.-M., Seifahrt, A., Madhusudhan, N., Chilingarian, I., Homeier, D., \& Szentgyorgyi, A. 2013, ApJ, 771,108

Becklin, E. E., \& Zuckerman, B. 1988, Nature, 336, 656

Beerer, I. M., et al. 2011, ApJ, 727, 23
Beichmann, C. A., Woolf, N. J., \& Lindensmith, C. A., The Terrestrial Planet Finder (TPF): A NASA Origins Program to Search for Habitable Planets, JPL publication 99-3 (Pasadena: Jet Propulsion Laboratory)

Belu, A. R., Selsis, F., Morales, J.-C., Ribas, I., Cossou, C., \& Rauer, H. 2011, A\&A, 525, A83

Benneke, B., \& Seager, S. 2012, ApJ, 753, 100

Benneke, B., \& Seager, S. 2013, ApJ, 778, 153

Berdyugina, S. V., Berdyugin, A. V., Fluri, D. M., \& Piirola, V. 2008, ApJ, 673, L83

Berdyugina, S. V., Berdyugin, A. V., Fluri, D. M., \& Piirola, V. 2011, ApJL, 728, L6

Berta, Z. K., et al. 2012, ApJ, 747, 35

Bezuit, J.-L., et al. 2010, in Pathways Towards Habitable Planets, ASP Conf. Series Vol. 430, ed. Coudé, du Foresto, V., Gelino, D. M., \& Ribas, I., 231

Birkby, J. L., de Kok, R. J., Brogi, M., de Mooij, E. J. W., Schwarz, H., Albrecht, S., \& Snellen, I. A. G. 2013, MNRAS, 436, L35

Blecic, J., et al. 2014, ApJ, 781, 116

Blecic, J., et al. 2013, ApJ, 779, 5

Bochanski, J. J., Burgasser, A. J., Simcoe, R. A., \& West, A. A. 2011, ApJ, 142, 169

Boeshaar, P. 1976, PhD thesis, Ohio State University

Boeshaar, P., \& Tyson, J. A. 1985, AJ, 90, 817

Bonfils, X., et al. 2013, A\&A, 556, A110

Borucki, W. J., et al. 2012, ApJ, 745, 120

Borucki, W. J., et al. 2013, Science, 340, 587

Borysow, A. 2002, A\&A, 390, 779

Bott, K., Kedziora-Chudczer, L., \& Bailey, J. 2013, in Proc. 12th Australian Space Science Conference, ed. W. Short \& I. Cairns (Sydney: National Space Society of Australia, Sydney), 41

Bowler, B. P., Liu, M. C., Dupuy, T. J., \& Cushing, M. C. 2010, ApJ, 723, 850

Boss, A., Basri, G., Kumar, S. S., Liebert, J., Martin, E. L., Reipurth, B., \& Zinnecker, H. 2003, in Brown Dwarfs, IAU Symp., Vol. 211, ed. E. Martín, 529

Bracewell, R. N. 1978, Nature, 274, 780

Brogi, M., Snellen, I. A. G., de Kok, R. J., Albrecht, S., Birkby, J., \& de Mooij, E. J. W. 2012, Nature, 486, 502

Brogi, M., Snellen, I. A. G., de Kok, R. J., Albrecht, S., Birkby, J. L., \& de Mooij, E. J. W. 2013, ApJ, 767, 27

Brogi, M., de Kok, R. J., Birkby, J. L., Schwarz, H., Snellen, I. A. G. 2014, A\&A, 565, A124

Buchwitz, M., et al. 2005, ACP, 5, 3313

Buenzli, E., \& Schmid, H. M. 2009, A\&A, 504, 259

Buenzli, E., et al. 2012, ApJL, 760, L31

Burgasser, A. J., et al. 2002, ApJ, 564, 421

Burgasser, A. J., Kirkpatrick, J. D., Liebert, J., \& Burrows, A. 2003, ApJ, 594, 510

Burgasser, A., McElwain, M. W., Kirkpatrick, J. D., Cruz, K. L., Tinney, C. G., \& Reid, I. N. 2004, AJ, 127, 2856

Burgasser, A. J., Geballe, T. R., Leggett, S. K., Kirkpatrick, J. D., \& Golimowski, D. A. 2006, ApJ, 637, 1067

Burgasser, A. J., Kirkpatrick, J. D., Cruz, K. L., Reid, I. N., Leggett, S. K., Liebert, J., Burrows, A., \& Brown, M. E. 2006b, ApJS, 166,585

Burgasser, A. J., Sheppard, S. S., \& Luhman, K. L. 2013, ApJ, 772, 129

Burningham, B., et al. 2008, MNRAS, 391, 320

Burningham, B., et al. 2010, MNRAS, 406, 1885

Burningham, B., et al. 2013, MNRAS, 433, 457 
Burrows, A., \& Sharp, C. M. 1999, ApJ, 512, 843

Burrows, A., \& Volobuyev, M. 2003, ApJ, 583, 985

Burrows, A., et al. 1997, ApJ 491, 856

Burrows, A., Hubbard, W. B., Lunine, J. I., \& Liebert, J. 2001, RvMP, 73, 719

Burrows, A., Sudarsky, D., \& Hubeny, I. 2003, ApJ, 588, 1121

Burrows, A., Sudarsky, D., \& Lunine, J. I. 2003, ApJ, 596, 587

Burrows, A., Sudarsky, D., \& Hubeny, I. 2006, ApJ, 640, 1063

Burrows, A., Hubeny, I., Budaj, J., \& Hubbard, W. B. 2007a, ApJ, 661,502

Burrows, A., Hubeny, I., Budaj, J., Knutson, H. A., \& Charbonneau, D. 2007b, ApJL, 668, L171

Burton, J. R., Watson, C. A., Littlefair, S. P., Dhillon, V. S., Gibson, N. P., Marsh, T. R., \& Pollacco, D. 2012, ApJS, 201, 36

Cáceres, C., et al. 2011, A\&A, 530, A5

Campargue, A., Leshchishina, O., Wang, L., Mondelain, D., Kassi, S., \& Nikitin, A.V. 2012a, JQSRT, 113, 1855

Campargue, A., et al. 2012b, Icar, 219, 110

Campargue, A., Leshchishina, O., Wang, L., Mondelain, D., \& Kassi, S. 2013, JMoSp, 291, 16

Campo, C. J., et al. 2011, ApJ, 727, 125

Cannon, A. J., \& Pickering, E. C. 1901, Ann. Astron. Obs. Harvard Coll., 28(II), 131

Carpenter, J. M. 2001, AJ, 121, 2851

Cash, W. 2006, Nature, 442, 51

Castelli, F., \& Kurucz, R. L. 2004, arXiv:astro-ph/0405087

Chabrier, G., \& Baraffe, I. 1997, A\&A, 327, 1039

Chabrier, G., Baraffe, I., Allard, F., \& Hauschildt, P. H. 2000a, ApJ, 542,464

Chabrier, G., Baraffe, I., Allard, F., \& Hauschildt, P. 2000b, ApJ, 542, L119

Chandrasekhar, S. 1960, Radiative Transfer (New York: Dover)

Charbonneau, D., Noyes, R. W., Korzennik, S. G., Nisenson, P., Jha, S., Vogt, S. S., \& Kilbrick, R. I. 1999, ApJ, 522, L145

Charbonneau, D., Brown, T. M., Noyes, R. W., \& Gilliland, R. L. 2002, ApJ, 568, 377

Charbonneau, D., et al. 2005, ApJ, 626, 523

Charbonneau, D., Knutson, H. A., Barman, T., Allen, L. A., Mayor, M., Megeath, S. T., Queloz, D., \& Udry, S. 2008, ApJ, 686, 1341

Charbonneau, D., et al. 2009, Nature, 462, 891

Chase, M.W. 1998, J. Phys. Cham. Ref. Data Mongraph No 9, NISTJANAF Thermochemical Tables. American Chemical Society. Am. Inst. Phys., Woodbury

Chauvin, G., Lagrange, A.-M., Dumas, C., Zuckerman, B., Mouillet, D., Song, I., Beuzit, J.-L., \& Lawrence, P. 2005, A\&A, 438, L25

Chen, G., van Boekel, R., Madhusudhan, N., Wang, H., Nikolov, N., Seeman, U., \& Henning, T. 2014a, A\&A, 564, A6

Chen, G., van Boekel, R., Wang, H., Nikolov, N., Seemann, U., \& Henning, Th. 2014b, A\&A, 567, A8

Chilcote, J., et al. 2014, submitted to ApJL, arXiv:1407.4469

Christiansen, J. L., et al. 2010, ApJ, 710, 97

Clarke, F. J., Tinney, C. G., \& Covey, K. R. 2002, MNRAS, 332, 361

Clarke, F. J., Hodgkin, S. T., Oppenheimer, B. R., Robertson, J., \& Haubois, X. 2008, MNRAS, 386, 2009

Cockell, C. S., et al. 2009, AsBio, 9, 1

Collier Cameron, A., Horne, K., Penny, A., \& James, D. 1999, Nature, 402, 751
Collier Cameron, A., Horne, K., Penny, A., \& Leigh, C. 2002, MNRAS 330, 187

Cooper, C. S., \& Showman, A. P. 2005, ApJ, 629, L45

Cooper, C. S., \& Showman, A. P. 2006, ApJ, 649, 1048

Cotton, D. V., Bailey, J., Crisp, D., \& Meadows, V. S. 2012, Icar, 217,570

Cowan, N. B., Agol, E., \& Charbonneau, D. 2007, MNRAS, 379, 641

Cowan, N. B., et al. 2009, ApJ, 700, 915

Cowan, N. B., Abbot, D. S., \& Voigt, A. 2012a, ApJL, 752, L3

Cowan, N. B., Machalek, P., Croll, B., Shekhtman, L. M., Burrows, A., Deming, D., Greene, T., \& Hora, J. L. 2012b, ApJ, 747, 82

Cowan, N. B., \& Strait, T. E. 2013, ApJL, 765, L17

Croll, B., Jayawardhana, R., Fortney, J. J., Lafrenière, D., \& Loic, A. 2010a, ApJ, 718, 920

Croll, B., Loic, A., Lafreniere, D., Jayawardhanan, R., \& Fortney, J. J. 2010b, ApJ, 717, 1084

Croll, B., Lafreniere, D., Loic, A., Jayawardhana, R., Fortney, J., \& Murray, N. 2011, AJ, 141, 30

Crossfield, I. J. M., Hansen, B. M. S., Harrington, J., Cho, J. Y.-K., Deming, D., Menou, K., \& Seager, S. 2010, ApJ, 723, 1436

Crossfield, I. J. M., Barman, T., \& Hansen, B. M. S. 2011, ApJ, 736, 132

Crossfield, I. J. M., Barman, T., Hansen, B. M. S., Ichi, T., \& Kodama, T. 2012b, ApJ, 760, 140

Crossfield, I. J. M., Knutson, H., Fortney, J., Showman, A. P., Cowan, N. B., \& Deming, D. 2012a, ApJ, 752, 81

Crossfield, I. J. M., Hansen, B. M. S., \& Barman, T. 2012c, ApJ, 746,46

Crossfield, I. J. M., Barman, T., Hansen, B. M. B., \& Howard, A. W. 2013, A\&A, 559, A33

Crossfield, I. J. M., et al. 2014, Nature, 505, 654

Crouzet, N., McCullough, P. R., Burke, C., \& Long, D. 2012, ApJ, 761, 7

Crouzet, N., McCullough, P. R., Deming, D., \& Madhusudhan, N. 2014, ApJ, in press, arXiv: 1409.4000

Cubillas, P., et al. 2013, ApJ, 768, 42

Cushing, M. C., Rayner, J. T., \& Vacca, W. D. 2005, ApJ, 623, 1115

Cushing, M. C., et al. 2011, ApJ, 743, 50

Dahn, C. C., et al. 2002, AJ, 124, 1170

Daumont, L., et al. 2013, JQSRT, 116, 101

de Bergh, C., et al. 2012, P\&SS, 61, 85

de Kok, R. J., Helling, Ch., Stam, D. M., Woitke, P., \& Witte, S. 2011, A\&A, 531, A67

de Kok, R. J., Brogi, M., Snellen, I. A. G., Birkby, J., Albrecht, S., \& de Mooij, E. W. J. 2013, A\&A, 554, 82

de Mooij, E. J. W., \& Snellen, I. A. G. 2009, A\&A, 493, L35

de Mooij, E. J. W., de Kok, R. J., Nefs, S. V., \& Snellen, I. A. G. 2011, A\&A, 528, A49

de Mooij, E. J. W., Brogi, M., de Kok, R. J., Snellen, I. A. G., Kenworthy, M., \& Karjaleinen, R. 2012, A\&A, 550, A54

de Wit, J., Gillon, M., Demory, B.-O., \& Seager, S. 2012, A\&A, 548, A128

Delfosse, X., et al. 1997, A\&A, 327, 25

Delorme, P., et al. 2008a, A\&A, 484, 469

Delorme, P., et al. 2008b, A\&A, 482, 961

Delorme, P., et al. 2012, A\&A, 548, A26

Deleuil, M., et al. 2014, A\&A, 564, A56 
Deming, D., Seager, S., Richardson, L. J., \& Harrington, J. 2005, Nature, 434, 740

Deming, D., Harrington, J., Seager, S., \& Richardson, J. L. 2006, ApJ, 644, 560

Deming, D., Harrington, J., Laughlin, G., Seager, S., Navarro, S. B., Bowman, W. C., \& Horning, K. 2007, ApJ, 667, L199

Deming, D., et al. 2011, ApJ, 726, 95

Deming, D., et al. 2012, ApJ, 754, 106

Deming, D., et al. 2013, ApJ, 774, 95

Demory, B.-O., \& Seager, S. 2011, ApJS, 197, 12

Demory, B.-O., et al. 2011, ApJL, 735, L12

Demory, B.-O., et al. 2013, ApJL, 776, L25

Des Marais, D. J., et al. 2002, AsBio, 2, 153

Désert, J.-M., et al. 2011a, ApJS, 197, 11

Désert, J.-M., et al. 2011b, ApJS, 197, 14

Dobbs-Dixon, I., \& Lin, D. N. C. 2008, ApJ, 673, 513

Dobbs-Dixon, I., \& Agol, E. 2013, MNRAS, 435, 3159

Dupuy, T. J., \& Liu, M. C. 2012, ApJS, 201, 19

Dupuy, T. J., \& Kraus, A. L. 2013, Science, 341, 1492

Ehrenreich, D., et al. 2014, A\&A, in press, arXiv:1405.1056

Esteves, L. J., de Mooij, E. J. W., \& Jayawardhana, R. 2013, ApJ, 772,51

Evans, K. F. 1998, JAtS, 55, 429

Evans, T. M., et al. 2013, ApJL, 772, L16

Fan, X. et al. 2000, AJ, 119, 928

Feroz, F., \& Hobson, M. 2014, MNRAS, 437, 3540

Flasar, F. M., et al. 2005, Science, 307, 1247

Flectcher, L. N., Baines, K. H., Momary, T. W., Showman, A. P., Irwin, P. G. J., Orton, G. S., Roos-Serote, M., \& Merlet, C. 2011, Icar, 214, 510

Freedman, R. S., Marley, M. S., \& Lodders, K. 2008, ApJS, 174, 504

Fressin, F., Knutson, H. A., Charbonneau, D., O’Donovan, F. T., Burrows, A., Deming, D., Mandushev, G., \& Spiegel, D. 2010, ApJ, 711, 374

Fressin, F., et al. 2013, ApJ, 766, 81

Ford, E. B., Seager, S., \& Turner, E. L. 2001, Nature, 412, 885

Forget, F., \& Pierrehumber, R. T. 1997, Science, 278, 1273

Fortney, J. J., Lodders, K., Marley, M. S., \& Freedman, R. S. 2008, ApJ, 678, 1419

Fortney, J. J., et al. 2011, ApJS, 197, 9

Fossati, L., et al. 2010, ApJL, 714, L222

Fujii, Y., Kawahara, H., Yasushi, S., Fukuda, S., Nakajima, T., Livengood, T. A., \& Turner, E. L. 2011, ApJ, 738, 184

Garnier, A., Pelon, J., Dubuisson, P. Faivre, M., Chomette, O., Pascal, N., \& Kratz, D. P. 2012, JAMS, 51, 1407

Geballe, T. R., Saumon, D., Leggett, S. K., Knapp, G. R., Marley, M. S., \& Lodders, K. 2001, ApJ, 556, 373

Geballe, T. R., et al. 2002, ApJ, 564, 466

Gibson, N. P., et al. 2010, MNRAS, 404, L114

Gibson, N. P., Pont, F., \& Aigrain, S. 2011, MNRAS, 411, 2199

Gibson, N. P., et al. 2012, MNRAS, 422, 753

Gibson, N. P., Aigrain, S., Barstow, J. K., Evans, T. M., Fletcher, L. N., \& Irwin, P. G. J. 2013a, MNRAS, 436, 2974

Gibson, N. P., Aigrain, S., Barstow, J. K., Evans, T. M., Fletcher, L. N., \& Irwin, P. G. J. 2013b, MNRAS, 428, 3680

Gillon, M., et al. 2009, A\&A, 506, 359

Gillon, M., et al. 2010, A\&A, 511, A3

Gillon, M., Triaud, A. H. M. J., Jehin, E., Delrez, L., Opitom, C., Magain, P., Lendl, M., \& Queloz, D. 2013, A\&A, 555, L5

Golimowski, D. A., et al. 2004, ApJ, 127, 3516
Goody, R., West, R., Chen, L., \& Crisp, D. 1989, JQSRT, 42, 539

Gray, D. F. 2005, The Observation and Analysis of Stellar Photospheres (3rd edn; Cambridge: Cambridge University Press)

Greathouse, T. K., Richter, M., Lacy, J., Moses, J., Orton, G., Encrenaz, T., Hammel, H. B., \& Jaffe, D. 2011, Icar, 214, 606

Gregory, P. C. 2012, MNRAS, 415, 2523

Grevesse, N., Asplund, M., \& Sauval, A. J. 2007, SSRv, 130, 15

Grillmair, C. J., et al. 2008, Nature, 457, 767

Guillot, T., \& Showman, A. P. 2002, A\&A, 385, 156

Gustaffson, B., Edvardsson, B., Eriksson, K., Jørgensen, U. G., Nordlund, A., \& Plez, B. 2008, A\&A, 486, 951

Hamdani, S., et al. 2006, A\&A, 460, 617

Hansen, J. E., \& Hovenier, J. W. 1974, JAtS, 31, 1137

Hargreaves, R. J., Hinkle, K. H., Bauschlicher, C. W., Wende, S., Seifahrt, A., \& Bernath, P. F. 2010, AJ, 140, 919

Hargreaves, R. J., Li, G., \& Bernath, P. F. 2011, ApJ, 735, 111

Hargreaves, R. J., Li, G., \& Bernath, P. F. 2012a, JQSRT, 113, 670

Hargreaves, R. J., Beale, C. A., Michaux, L., Irfan, M., \& Bernath, P. F. 2012b, ApJ, 757, 46

Hauschildt, P. H., \& Baron, E. 1999, JCoAM, 109, 41

Hawley, S. L., et al. 2002, AJ, 123, 3049

Hayashi, C., \& Nakano, T. 1963, PThPh, 30, 460

Heinze, A. N., et al. 2013, ApJ, 767, 173

Helling, Ch., \& Woitke, P. 2006, A\&A, 455, 325

Helling, Ch., Dehn, M., Woitke, P., \& Hauschildt, P. H. 2008a, ApJL, 675, L105

Helling, Ch., et al. 2008b, MNRAS, 391, 1854

Heng, K., Frierson, D. M. W., \& Phillips, P. J. 2011, MNRAS, 418, 2669

Henyey, L., Vardya, M. S., \& Bodenheimer, P. 1965, ApJ, 142, 841

Hesman, B. E., et al. 2009, Icar, 202, 249

Hilico, J. C., Robert, O., Loete, M., Tuomi, S., Pine, A. S., \& Brown, L. R. 2001, JMoSp, 208, 1

Hough, J. H., Lucas, P. W., Bailey, J. A., Tamura, M., Hirst, E., Harrison, D., \& Bartholomew-Biggs, M. 2006, PASP, 118, 1302

Howard, A. W., et al. 2010, Science, 330, 653

Howard, A. W., et al. 2012, ApJS, 201, 15

Huang, X., Gamache, R. R., Freedman, R. S., Schwenke, D. W., \& Lee, T. J. 2013, JQSRT, 130, 134

Huang, X., Freedman, R. S., Tashkun, S. A., Schwenke, D. W., \& Lee, T. J. 2014, JQSRT, 147, 134

Hubeny, I., Burrows, A., \& Sudarsky, D. 2003, ApJ, 594, 1011

Huitson, C. M., Sing, D. K., Vidal-Madjar, A., Ballester, G. E., Lecavelier des Etangs, A., Désert, J.-M., \& Pont, F. 2012, MNRAS, 422, 2477

Huitson, C. M., et al. 2013, MNRAS, 434, 3252

Ibgui, L., Burrows, A., \& Spiegel, D. S. 2010, ApJ, 713, 751

Irwin, P. G. J., Teanby, N. A., \& Davis, G. R. 2010, Icar, 208, 913

Irwin, P. G. J., et al. 2012, Icar, 220, 369

Jackson, B., Greenberg, R., \& Barnes, R. 2008, ApJ, 681, 1631

Janson, M., et al. 2013, ApJL, 778, L4

Jensen, A. G., Redfield, S., Endl, M., Cochran, W. D., Koesterke, L., \& Barman, T. S. 2011, ApJ, 743, 203

Jensen, A. G., Redfield, S., Endl, M., Cochran, W. D., Koesterke, L., \& Barman, T. S. 2012, ApJ, 751, 86

Jordán, A., et al. 2013, ApJ, 778, 184

Kaltenegger, L., \& Traub, W. A. 2009, ApJ, 698, 519

Kane, S. R., Barclay, T., \& Gelino, D. M. 2013, ApJL, 770, L20

Karalidi, T., Stam, D. M., \& Hovenier, J. W. 2012, A\&A, 548, A90 
Kasper, M., et al. 2010, in Ground-based and Airborne Instrumentation for Astronomy III, Proc. SPIE, Vol. 7735, ed. I. S. McLean, S. K. Ramsay, \& H. Takami, 77352E

Kasting, J. F. 1988, Icar, 74, 472

Kasting, J. F., Whitmire, D. P., \& Reynolds, R. T. 1993, Icar, 101, 108

Kawahara, H., \& Fujii, Y. 2010, ApJ, 720, 1333

Kedziora-Chudczer, L., \& Bailey, J. 2011, MNRAS, 414, 1483

Keller, C. U. 2006, in Ground-based and Airborne Instrumentation for Astronomy, SPIE Proc. Vol. 6269, ed. I. S. MClean, \& M. Iye (Bellingham: SPIE), 26

Kemp, J. C., Henson, G. D., Steiner, C. T., \& Powell, E. R. 1987, Nature, 326, 270

Kiang, N. Y., et al. 2007, AsBio, 7, 252

Kipping, D., \& Bakos, G. 2011, ApJ, 730, 50

Kipping, D., \& Spiegel, D. S. 2011, MNRAS, 417, L88

Kirkpatrick, J. D., Henry, T. J., \& McCarthy, D. W. 1991, ApJS, 77, 417

Kirkpatrick, J. D., et al. 1999, ApJ, 519, 802

Kirkpatrick, J. D., et al. 2000, AJ, 120, 447

Kirkpatrick, J. D., et al. 2010, ApJS, 190, 100

Kirkpatrick, J. D., et al. 2011, ApJS, 197, 19

Kirkpatrick, J. D., et al. 2012, ApJ, 753, 156

Kniazev, A. Y., et al. 2013, ApJ, 770, 124

Knutson, H. A., et al. 2007a, Nature, 447, 183

Knutson, H. A., Charbonneau, D., Noyes, R. W., Brown, T. M., \& Gilliland, R. L. 2007b, ApJ, 655, 564

Knutson, H. A., Charbonneau, D., Allen, L. E., Burrows, A., \& Megeath, S. T. 2008, ApJ, 673, 526

Knutson, H. A., Charbonneau, D., Burrows, A., O’Donovan, F. T., \& Mandushev, G. 2009a, ApJ, 691, 866

Knutson, H. A., et al. 2009b, ApJ, 690, 822

Knutson, H. A., Charbonneau, D., Cowan, N. B., Fortney, J. J., Showman, A. P., Agol, E., \& Henry, G. W. 2009c, ApJ, 703, 769

Knutson, H. A., Howard, A. W., \& Isaacson, H. 2010, ApJ, 720, 1569

Knutson, H. A., et al. 2012, ApJ, 754, 22

Knutson, H. A., Benneke, B., Deming, D., \& Homeier, D. 2014a, Nature, 505, 66

Knutson, H. A., et al. 2014b, submitted to ApJ, arXiv:1403.4602

Koen, C. 2006, MNRAS, 367, 1735

Konopacky, Q. M., Barman, T. S., Macintosh, B. A., \& Marois, C. 2013, Sci, 339, 1398

Kopparapu, R. K., et al. 2013, ApJ, 765, 131

Kopparapu, R. K., Ramirez, R. M., SchottelKotte, J., Kasting, J. F., Domagal-Goldman, S., \& Eymet, V. 2014, ApJ, 787, L29

Kreidberg, L., et al. 2014a, Nature, 505, 69

Kreidberg, L., et al. 2014b, ApJ, 793, L27

Kumar, S. S. 1963, ApJ, 137, 1121

Kurucz, R. 1970, SAO Special Report 309, Atlas: a Computer Program for Calculating Model Stellar Atmospheres (Cambridge, MA: Smithsonian Astrophysical Observatory)

Kurucz, R. 1993, PhST, T47, 110

Kuzuhara, M., et al. 2013, ApJ, 774, 11

Lane, C., et al. 2007, ApJ, 668, L163

Langford, S. V., Wyithe, J. S. B., \& Turner, E. L. 2009, AsBio, 9, 305

Langford, S. V., Wyithe, J. S. B., Turner, E. L., Jenkins, E. B., Narita, N., Liu, X., Suto, Y., \& Toru, Y. 2011, MNRAS, 415, 673
Lecavelier des Etangs, A., et al. 2010, A\&A, 514, A72

Leconte, J., Forget, F., Charnay, B., Wordsworth, R., \& Pottier, A. 2013, Nature, 504, 268

Lee, J.-M., Fletcher, L. N., \& Irwin, P. G. J. 2012, MNRAS, 420, 170

Lendl, M., Gillon, M., Queloz, D., Alonso, R., Fumel, A., Jehin, E., \& Naef, D. 2013, A\&A, 552, 11

Leggett, S. K. 1992, ApJS, 82, 351

Leggett, S. K., et al. 2000, ApJ, 536, 35

Leggett, S. K., Allard, F., Geballe, T. R., Hauschildt, P. H., \& Schweitzer, A. 2001, ApJ, 548, 908

Leggett, S. K., et al. 2002, ApJ, 564, 452

Leggett, S. K., Saumon, D., Marley, M. S., Geballe, T. R., Golimowski, D. A., Stephens, D., \& Fan, X. 2007, ApJ, 655, 1079

Leggett, S. K., et al. 2010, ApJ, 710, 1627

Leggett, S. K., et al. 2011, in Astronomical Society of the Pacific Conference Series, 16th Cambridge Workshop on Cool Stars, Stellar Systems and the Sun, Vol. 448, ed. C. Johns-Krull, 913

Leggett, S. K., Morley, C. V., Marley, M. S., Saumon, D., Fortney, J. J., \& Visscher, C. 2013, ApJ, 763, 130

Leigh, C., Collier Cameron, A., Horne, K., Penny, A., \& James, D. 2003a, MNRAS, 344, 1271

Leigh, C., Collier Cameron, A., Udry, S., Donati, J.-F., Horne, K., James, D., \& Penny, A. 2003b, MNRAS, 346, L16

Lindal, G. F. 1992, AJ, 103, 967

Line, M. R., Liang, M. C., \& Yung, Y. 2010, ApJ, 717, 496

Line, M. R., Vasischt, G., Chen, P., Angerhausen, D., \& Yung, Y. 2011, ApJ, 738, 32

Line, M. R., Zhang, X., Vasischt, G., Natraj, V., Chen, P., \& Yung, Y. 2012, ApJ, 749, 93

Line, M. R., Knutson, H., Deming, D., Wilkins, A., \& Desert, J.-M. 2013a, ApJ, 778, 183

Line, M. R., et al. 2013b, ApJ, 775, 137

Linsky, J. L., Yang, H., France, K., Froning, C. S., Green, J. C., Stocke, J. T., \& Osterman, S. N. 2010, ApJ, 717, 1291

Liou, K. N. 2002, An Introduction to Atmospheric Radiation (Sab Diego: Academic Press)

Liu, M. C., Leggett, S. K., Golimowski, D. A., Chiu, K., Fan, X., Geballe, T. R., Schneider, D. P., \& Brinkmann, J. 2006, ApJ, 647,1393

Livengood, T. A., et al. 2011, AsBio, 11, 907

Lockwood, A. C., Johnson, J. A., Bender, C. F., Carr, J. S., Barman, T., Richert, A. J. W., \& Blake, G. A. 2014, ApJL, 783, L29

Lodders, K. 2002, ApJ, 577, 974

Lodders, K., \& Fegley, B. 1994, Icar, 112, 368

Lodders, K., \& Fegley, B. 2002, Icar, 155, 393

Looper, D. L., Gelino, C. R., Burgasser, A. J., \& Kirkpatrick, J. D. 2008, ApJ, 685, 1183

López-Morales, M., Coughlin, J. L., Sing, D. K., Burrows, A., Apai, D., Rogers, J. C., Spiegel, D. S., \& Adams, E. R. 2010, ApJL, 716, L36

Lovelock, J. E. 1965, Nature, 207, 568

Lucas, P. W., \& Roche, P. F. 2000, MNRAS, 314, 858

Lucas, P. W., Hough, J. H., Bailey, J. A., Tamura, M., Hirst, E., \& Harrison, D. 2009, MNRAS, 393, 229

Lucas, P. W., et al. 2010, MNRAS, 408, L56

Luhman, K. L. 2013, ApJL, 767, L1

Luhman, K. L. 2014, ApJL, 768, L18

Luhman, K. L., Burgasser, A. J., \& Bochanski, J. J. 2011, ApJL, 730, L9 
Luhman, K. L., Burgasser, A. J., Labbé, I., Saumon, D., Marley, M. S., Bochanski, J. J., Monson, A. J., \& Pearson, S. E. 2012, ApJ, 744, 135

Mace, G. N., et al. 2013, ApJS, 205, 6

Machalek, P., McCullough, P. R., Burke, C. J., Valenti, J. A., Burrows, A., \& Hora, J. L. 2008, ApJ, 684, 1427

Machalek, P., McCullough, P. R., Burrows, A., Burke, C. J., Hora, J. L., \& Johns-Crull, C. M. 2009, ApJ, 701, 514

Machalek, P., Greene, T., McCullough, P. R., Burrows, A., Burke, C. J., Hora, J. L., Johns-Krull, C. M., \& Deming, D. 2010, ApJ, 711,111

Macintosh, B. A., et al. 2012, in Ground-based and Airborne Instrumentation for Astronomy IV, Proc. SPIE, Vol. 8446, ed. I. S. McLean, S. K. Ramsay, \& H. Takami, 84461U

Madhusudhan, N., \& Seager, S. 2009, ApJ, 707, 24

Madhusudhan, N., \& Seager, S. 2010, ApJ, 725, 261

Madhusudhan, N., et al. 2011, Nature, 469, 64

Madhusudhan, N. 2012, ApJ, 758, 36

Majeau, C., Agol, E., \& Cowan, N. B. 2012, ApJL, 747, L20

Mandell, A. M., Deming, L. D., Blake, G. A., Knutson, H. A., Mumma, M. J., Villanueva, G. L., \& Salyk, C. 2011, ApJ, 728, 18

Mandell, A. M., Haynes, K., Sinukoff, E., Madhusudhan, N., Burrows, A., \& Deming, D. 2013, ApJ, 779, 128

Mahtani, D. P., et al. 2013, MNRAS, 432, 693

Marcy, G. W., Butler, R. P., Williams, E., Bildsten, L., Graham, J. R., Ghez, A. M., \& Jernigan, J. G. 1997, ApJ, 481, 926

Marley, M. S., Seager, S., Saumon, D., Lodders, K., Ackerman, A. S., Freedman, R. S., \& Fan, X. 2002, ApJ, 568, 335

Marley, M. S., Saumon, D., \& Goldblatt, C. 2010, ApJL, 723, L117

Marsh, K. A., Wright, E. L., Kirkpatrick, J. D., Gelino, C. R., Cushing, M., Griffith, R. L., Skrutskie, M. F., \& Eisenhardt, P. R. 2013, ApJ, 762, 119

Marois, C., Macintosh, B., Barman, T., Zuckerman, B., Song, I., Patience, J., Lafreniere, D., \& Doyon, R. 2008, Sci, 322, 1348

Marois, C., Zuckerman, B., Konopacky, Q. M., Macintosh, B., \& Barman, T. 2010, Nature, 468, 1080

Martín, E. L., Delfosse, X., \& Guieu, S. 2004, AJ, 127, 449

Maxted, P. F. L., et al. 2013, MNRAS, 428, 2645

Mayne, N. J., et al. 2014, A\&A, 561, A1

Mayor, M., \& Queloz, D. 1995, Nature, 378, 355

Mayor, M., et al. 2009, A\&A, 507, 487

McCullough, P., Crouzet, N., Deming, D., \& Madhusudhan, N. 2014, ApJ, 791, 55

Ménard, F., Delfosse, X., \& Monin, J.-L. 2002, A\&A, 396, L35

Menou, K., \& Rauscher, E. 2009, ApJ, 700, 887

Miller, N., \& Fortney, J. J. 2011, ApJL, 736, L29

Mohanty, S., Jayawardhana, R., Huélamo, N., \& Mamajek, E. 2007, ApJ, 657, 1064

Morley, C. V., Fortney, J. J., Marley, M. S., Channon, V., Saumon, D., \& Leggett, S. K. 2012, ApJ, 756, 172

Moro-Martín, A., Reike, G. H., \& Su, K. Y. L. 2010, ApJL, 721, L199

Morton, T. D., \& Johnson, J. A. 2011, ApJ, 738, 170

Moses, J. I., et al. 2011, ApJ, 737, 15

Moses, J. I. 2014, PTRSL, 372, 20130073

Mountain, M., et al. 2009, Astro2010: the Astronomy and Astrophysics Decadal Survey, Technology Development Papers, no. 12
Moya, A., Amado, P. J., Barrado, D., García Hernández, A., Aberasturi, M., Montesinos, B., \& Aceituno, F. 2010, MNRAS, 405, L81

Murgas, F., Pall'e, E., Zapatero Osorio, M. R., Nortmann, L., Hoyer, S., \& Cabrera-Lavers, A. 2014, A\&A, 563, A41

Nakajima, T., Oppenheimer, B. R., Kulkarni, S. R., Golinowski, D. A., Matthews, K., \& Durrance, S. T. 1995, Nature, 378, 463

Nakajima, T., Tsuji, T., \& Yanagisawa, K. 2004, ApJ, 607, 499

Nikitin, A., Boudon, V., Wenger, C., Albert, S., Brown, L., Baurecker, S., \& Quack, M. 2013a, PCCP, 15, 10071

Nikitin, A. V., Rey, M., \& Tyuterev, V. G. 2013b, CPL, 565, 5

Nikolov, N., et al. 2014, MNRAS, 437, 46

Noll, K. S., Geballe, T. R., Leggett, S. K., \& Marley, M. S. 2000, ApJL, 541, L75

Nyemeyer, S., et al. 2011, ApJ, 742, 35

O’Donovan, F. T., Charbonneau, D., Harrington, J., Madhusudhan, N., Seager, S., Deming, D., \& Knutson, H. A. 2010, ApJ, 710, 1551

O’Rourke, J. G., et al. 2014, ApJ, 781, 109

Oppenheimer, B. R., Kulkarni, S. R., Matthews, K., \& Nakajima, T. 1995, Sci, 270, 1478

Oppenheimer, B. R., et al. 2013, ApJ, 768, 24

Owen, T., Mahaffy, P., Niemann, H. B., Atreya, S., Donahue, T., Bar-Nun, A., \& de Pater, I. 1999, Nature, 402, 269

Pallé, E., Zapatero Osorio, M. R., García Muñoz, A. 2011, ApJ, 728,19

Patience, J., King, R. R., de Rosa, R. J., \& Marois, C. 2010, A\&A, 517, A76

Patten, B. M., et al. 2006, ApJ, 651, 502

Perna, R., Menou, K., \& Rauscher, E. 2010, ApJ, 724, 313

Pepe, F., et al. 2011, A\&A, 534, A58

Petigura, E. A., Howard, A. W., \& Marcy, G. W. 2013, PNAS, 110, 19273

Pierrehumbert, R., \& Gaidos, E. 2011, ApJL, 734, L13

Pinfield, D. J., et al. 2008, MNRAS, 390, 304

Pollacco, D. L., et al. 2006, PASP, 118, 1407

Pont, F., Knutson, H., Gilliland, R. L., Moutou, C., \& Charbonneau, D. 2008, MNRAS, 385, 109

Pont, F., Sing, D. K., Gibson, N. P., Aigrain, S., Henry, G., \& Husnoo, N. 2013, MNRAS, 432, 2917

Quintana, E. V., et al. 2013, ApJ, 767, 137

Quintana, E. V., et al. 2014, Sci, 344, 277

Radigan, J., Jayawardhana, R., Lafreniere, D., Artigau, E., Marley, M., \& Saumon, D. 2012, ApJ, 750, 105

Rajpurohit, A. S., Reylé, C., Allard, F., Homeier, D., Schultheis, M., Bessell, M. S., \& Robin, A. C. 2013, A\&A, 556, A15

Ranjan, S., Charbonneau, D., Désert, J.-M., Madhusudhan, N., Deming, D., Wilkins, A., \& Mandell, A. V. 2014, 785, 148

Rauer, H., et al. 2013, submitted to ExA, arXiv:1310.0696

Rauscher, E., \& Menou, K. 2012, ApJ, 750, 96

Rauscher, E., \& Menou, K. 2013, ApJ, 764, 103

Rayner, J. T., Cushing, M. C., \& Vacca, W. D. 2009, ApJS, 185, 289

Rebolo, R., Martín, E. L., \& Magazzu, A. 1992, ApJ, 389, L83

Rebolo, R., Zapatero-Osorio, M. R., \& Martin, E. L. 1995, Nature, 377, 129

Rebolo, R., Martín, E. L., Basri, G., Marcy, G. W., \& ZapateroOsorio, M. R. 1996, ApJ, 469, L53

Redfield, S., Endl, M., Cochran, W. D., \& Koesterke, L. 2008, ApJL, 673, L87 
Reid, I. N., Burgasser, A. J., Cruz, K. L., Kirkpatrick, J. D., \& Gizis, J. E. 2001, AJ, 121, 1710

Rey, M., Nikitin, A. V., \& Tyuterev, V. G. 2013, JMoSp, 291, 85

Rey, M., Nikitin, A. V., \& Tyuterev, V. G. 2014, ApJ, 789, 2

Richard, C., et al. 2012, JQSRT, 113, 1276

Richardson, L. J., Deming, D., Horning, K., Seager, S., \& Harrington, J. 2007, Nature, 445, 892

Ricker, G. R., et al. 2014, submitted to Proc. SPIE, arXiv:1406.0151

Robertson, P., Mahadevan, S., Endl, M., Roy, A. 2014, Sci, 345, 440

Robie, R. A., \& Hemingway, B. S. 1995, UGSB, 2131

Robinson, T. D., Meadows, V. S., \& Crisp, D. 2010, ApJL, 721, L67

Robinson, T. D., et al. 2011, AsBio, 11, 393

Rodler, F., Kurster, M., \& Henning, T. 2008, A\&A, 485, 859

Rodler, F., Kurster, M., \& Henning, T. 2010, A\&A, 514, A23

Rodler, F., Lopez-Morales, M., \& Ribas, I. 2012, ApJL, 753, L25

Rodler, F., Kürster, M., \& Barnes, J. R. 2013, MNRAS, 432, 1980

Rogers, J. C., Apai, D., López-Morales, M., Sing, D. K., \& Burrows, A. 2009, ApJ, 707, 1707

Rogers, T. M., \& Showman, A. P. 2014, ApJL, 782, L4

Rossow, W. 1978, Icar, 36, 1

Rostron, J. W., et al. 2014, MNRAS, 441, 36666

Rowe, J. F., et al. 2008, ApJ, 689, 1345

Rosenkranz, P. W. 2001, ITGRS, 39, 2429

Ruiz, M. T., Leggett, S. K., \& Allard, F. 1997, ApJ, 491, 107

Sanromá, E., Pallé, E., Parenteau, M. N., Kiang, N. Y., GutiérrezNavarro, A. M., López, R., \& Montañés-Rodríguez, P. 2014, ApJ, 780, 52

Santerne, A., Bonomo, A. S., Hébrard, G., Deleuil, M., Moutou, C., Almenara, J.-M., Bouchy, F., \& Díaz, R. F. 2011, A\&A, 536, A70

Saumon, D., Marley, M. S., Lodders, K., \& Freedman, R. S. 2003, in Brown Dwarfs, Proc. IAU Symp. vol. 211, ed. E. Martín, 345

Saumon, D., \& Marley, M. S. 2008, ApJ, 689, 1327

Saumon, D., Hubbard, W. B., Burrows, A., Guillot, T., Lunine, J. I., \& Chabrier, G. 1996, ApJ, 460, 993

Saumon, D., Marley, M. S., Abel, M., Frommhold, L., \& Freedman, R. S. 2012, 750, 74

Schindler, T. L., \& Kasting, J. F. 2000, Icar, 145, 262

Schlawin, E., Zhao, M., Teske, J. K., Herter, T. 2014, ApJ, 783, 5

Schmid, H. M., et al. 2005, in Direct Imaging of Exoplanets: Science and Techniques, IAU Coll. Vol. 200, ed. C. Aime \& F. Vakili (Cambridge: Cambridge University Press), 165

Schneider, J., Dedieu, C., Le Sidaner, P., Savalle, R., \& Zolotukhin, I. 2011, A\&A, 532, A79

Schweitzer, A., Gizis, J. E., Hauschildt, P. H., Allard, F., Howard, E. M., \& Kirkpatrick, J. D. 2002, ApJ, 566, 435

Seager, S., Whitney, B. A., \& Sasselov, D. D. 2000, ApJ, 540, 504

Seager, S., et al. 2014, Exo-S Science and Technology Development Team Interim Report (Pasadena: Jet Propulsion Laboratory)

Segura, A., et al. 2005, AsBio, 5, 706

Segura, A., Meadows, V. S., Kasting, J. F., Crisp, D., \& Cohen, M. 2007, A\&A, 472, 665

Seiff, A., et al. 1998, JGR, 103, E10, 22857

Selsis, F., Despois, D., \& Parisot, J.-P. 2002, A\&A, 388, 985

Selsis, F., Kasting, J. F., Levrard, B., Paillet, J., Ribas, I., \& Delfosse, X. 2007, A\&A, 476, 1373

Sengupta, S., \& Marley, M. S. 2010, ApJL, 722, L142

PASA, 31, e043 (2014)

doi:10.1017/pasa.2014.38
Shabram, M., Fortney, J. J., Greene, T. P., \& Freedman, R. S. 2011, ApJ, 727, 65

Sharp, C. M., \& Huebner, W. F. 1990, ApJ, 72, 417

Sharp, C. M., \& Burrows, A. 2007, ApJS, 168, 140

Showman, A. P., \& Guillot, T. 2002, A\&A, 385, 166

Showman, A. P., \& Polvani, L. M. 2011, ApJ, 738, 71

Showman, A. P., Fortney, J. J., Lian, Y., Marley, M. S., Freedman, R. S., Knutson, H. A., \& Charbonneau, D. 2009, ApJ, 699, 564

Showman, A. P., Cho, J. Y.-K., \& Menou, K. 2010, in Exoplanets, ed. S. Seager (University Arizona Press), 471

Showman, A. P., \& Kaspi, Y. 2013, ApJ, 776, 85

Shporer, A., et al. 2014, ApJ, 788, 92

Simons, D. A., \& Tokunaga, A. 2002, PASP, 114, 169

Sing, D. K., Vidal-Madjar, A., Désert, J. M., Lecavelier des Etangs, A., \& Ballester, G. 2008, ApJ, 686, 658

Sing, D. K., Désert, J.-M., Lecavelier des Etangs, A., Ballester, G. E., Vidal-Madjar, A., Parmentier, V., Hebrard, G., \& Henry, G. W. 2009, A\&A, 505, 891

Sing, D. K., et al. 2011a, MNRAS, 416, 1443

Sing, D. K., et al. 2011b, A\&A, 527, A73

Sing, D. K., et al. 2012, MNRAS, 426, 1663

Sing, D. K., et al. 2013, MNRAS, 436, 2956

Skemer, A., et al. 2014, ApJ, 792, 17

Smith, A. M. S., Anderson, D. R., Skillen, I., Collier-Cameron, A., \& Smalley, B. 2011, MNRAS, 416, 2096

Smith, A. M. S., et al. 2012, A\&A, 545, 93

Snellen, I. A. G., de Mooij, E. J. W., \& Albrecht, S. 2007, Nature, 459,543

Snellen, I. A. G., Albrecht, S., de Mooij, E. J. W., \& Le Poole, R. S. 2008, ApJ, 487, 357

Snellen, I. A. G., de Mooij, E. J. W., \& Albrecht, S. 2009, Nature, 459,543

Snellen, I. A. G., de Kok, R. J., de Mooij, E. J. W., \& Albrecht, S. 2010a, Nature, 465, 1049

Snellen, I. A. G., de Mooij, E. J. W., \& Burrows, A. 2010b, A\&A, 513, A76

Snellen, I., de Kok, R., Le Poole, R., Brogi, M., \& Birkby, J. 2013, ApJ, 764, 182

Snellen, I., Brandl, B. R., de Kok, R. J., Brogi, M., Birkby, J., \& Schwarz, H. 2014, Nature, 509, 63

Sorahana, S., \& Yamamura, I. 2012, ApJ, 760, 151

Soter, S. 2006, AJ, 132, 2513

Soummer, R., et al. 2009, in Techniques and Instrumentation for Detection of Exoplanets IV, Proc. SPIE Vol. 7440, ed. S. B. Shaklan, 74400A

Spergel, D., et al. 2013, WFIRST-AFTA Science Definition Team Final Report, IPAC, Pasadena

Spurr, R. J. D. 2001, JQSRT, 68, 689

Stam, D. M., \& Hovenier, J. W. 2005, A\&A, 444, 275

Stamnes, K., Tsay, S. C., Wiscombe, W., \& Jayaweera, K. 1988, ApOpt, 27, 2502

Stapelfeldt, K. R. 2005, in Scientific Requirements for Extremely Large Telescopes, ed. P. Whitelock, M. Dennefeld, \& B. Leibundgut, IAU Symp., No 232, 149

Stapelfeldt, K. 2014, Exo-C Science and Technology Definition Team Interim Report (Pasadena: Jet Propulsion Laboratory)

Stephens, D. C., \& Leggett, S. K. 2004, PASP, 116, 9

Stephens, D. C., et al. 2009, ApJ, 702, 154

Sterzik, M. F., Bagnulo, S., \& Palle, E. 2012, Nature, 483, 64

Stevenson, K. B., et al. 2010, Nature, 464, 1161

Stevenson, K. B., et al. 2012, ApJ, 754, 136 
Stevenson, K. B., et al. 2014, AJ, 147, 161

Sudarsky, D., Burrows, A., \& Pinto, P. 2000, ApJ, 538, 885

Swain, M. R., Vasisht, G., \& Tinetti, G. 2008, Nature, 452, 329

Swain, M. R., Vasischt, G., Tinetti, G., Bouwman, J., Chen, P., Yung, Y., Deming, D., \& Deroo, P. 2009a, ApJL, 690, L114

Swain, M. R., et al. 2009, ApJ, 704, 1616

Swain, M. R., et al. 2010, Nature, 463, 637

Swain, M. R., et al. 2013, Icar, 225, 432

Sudol, J. J., \& Haghighipour, N. 2012, ApJ, 755, 38

Takahashi, J., Itoh, Y., Akitaya, H., Okazaki, A., Kawabata, K., Oasa, Y., \& Isogai, M. 2013, PASJ, 65, 38

Tarter, J. 1975, PhD thesis, California University, Berkeley

Tashkun, S. A., Perevalov, V. I., Teffo, J. -L., Bykov, A. D., \& Lavrentieva, N. N. 2003, JQSRT, 82, 165

Tata, R., Martín, E. L., Sengupta, S., Phan-Bao, N., Zapatero Osorio, M. R., \& Bouy, H. 2009, 508, 1423

Tennyson, J., \& Yurchenko, S. N. 2012, MNRAS, 425, 21

Tinetti, G., Meadows, V. S., Crisp, D., Fong, W., Fishbein, E., Turnbull, M., \& Bibring, J.-P. 2006, AsBio, 6, 34

Tinetti, G., et al. 2010, ApJL, 712, L139

Tinetti, G., et al. 2012, ExA, 34, 311

Tinney, C. G., Burgasser, A. J., \& Kirkpatrick, J. D. 2005, AJ, 126 , 975

Tinney, C. G., Faherty, J. K., Kirkpatrick, J. D., Wright, E. L., Gelino, C. R., Cushing, M. C., Griffith, R. L., \& Salter, G. 2012, ApJ, 759, 60

Todorov, K. O., Deming, D., Harrington, J., Stevenson, K. B., Bowman, W. C., Nymeyer, S., Fortney, J. J., \& Bakos, G. A. 2010, ApJ, 708, 498

Todorov, K. O., et al. 2012, ApJ, 746, 111

Todorov, K. O., et al. 2013, ApJ, 770, 102

Traub, W. A., et al. 2006, in Advances in Stellar Interferometry, Proc. SPIE, Vol. 6268, ed. J. D. Monnier, M. Scholler, \& W. C. Danchi, 62680T

Tsuji, T. 1973, A\&A, 23, 411

Tsuji, T. 2002, ApJ, 575, 264

Tsuji, T. 2005, ApJ, 621, 1033

Tsuji, T., \& Nakajima, T. 2003, ApJ, 585, L151

Tsuji, T., Yamamura, I., \& Sorahana, S. 2011, ApJ, 734, 73

Tsuji, T., Ohnaka, K., Aoki, W., \& Nakajima, T. 1996, A\&A, 308, L29

Tuomi, M., et al. 2012, A\&A, 551, A79

Tuomi, M., et al. 2013, A\&A, 549, A48

Turnbull, M. C., et al. 2012, PASP, 124, 418

Venot, O., Hébrard, E., Agúndez, M., Dobrijevic, M., Selsis, F, Hersant, F, Iro, N., Bounaceur, R. 2012, A\&A, 546, A43

Vidal-Madjar, A., Lecavelier des Etangs, A., Désert, J.-M., Ballester, G. E., Ferlet, R., Hébrard, G., \& Mayor, M. 2003, Nature, 422, 143

Vidal-Madjar, A., et al. 2004, ApJL, 604, L69

Vogt, S. S., Butler, R. P., Rivera, E. J., Haghighipour, N., Henry, G. W., \& Williamson, M. H. 2010, ApJ, 723, 954

Vrba, F. J., et al. 2004, ApJ 127, 2948

Wakeford, H. R., et al. 2013, MNRAS, 435, 3481

Waldmann, I. P., Tinetti, G., Drossart, P., Swain, M. R., Deroo, P., \& Griffith, C. A. 2012, ApJ, 744, 35

Wang, L., Mondelain, D., Kassi, S., \& Campargue, A. 2012, JQSRT, 113,47

Wang, W., van Boekel, R., Madhusudhan, N., Chen, G., Zhao, G., \& Henning, Th. 2013, ApJ, 770, 70
Warren, S. J., et al. 2007, MNRAS, 381, 1400

Weiss, L. M., et al. 2013, ApJ, 768, 14

Welsh, W. F., et al. 2010, ApJL, 713, L145

Wenger, C., \& Champion, J. P. 1998, JQSRT, 59, 471

Wiktorowicz, S. J. 2009, ApJ, 696, 1116

Wiktorowicz, S. J., \& Matthews, K. 2008, PASP, 120, 1282

Wilkins, A., Deming, D., Madhusushan, N., Burrows, A., Knutson, H., McCullough, P., \& Ranjan, S. 2014, ApJ, 783, 113

Williams, D. M., \& Gaidos, E. 2008, Icar, 195, 927

Williams, P. K. G., Charbonneau, D., Cooper, C. S., Showman, A. P., \& Fortney, J. J. 2006, ApJ, 649, 1020

Wittenmyer, R. A., Tinney, C. G., Butler, R. P., O’Toole, S. J., Jones, H. R. A., Carter, B. D., Bailey, J., \& Horner, J. 2011, ApJ, 738, 81

Wittenmyer, R. A., et al. 2014, ApJ, 791, 114

Woitke, P., \& Helling, Ch. 2003, A\&A, 399, 297

Woitke, P., \& Helling, Ch. 2004, A\&A, 414, 335

Wolf, E. T., \& Toon, O. B. 2014, GeoRL, 41, 167

Wong, M. H., Mahaffy, P. R., Atreya, S. K., Niemann, H. B., \& Owen, T. C. 2004, Icar, 171, 153

Wood, P. L., Maxted, P. F. L., Smalley, B., \& Iro, N. 2011, MNRAS, 412, 2376

Woolf, N. J., Smith, P. S., Traub, W. A., \& Jucks, K. W. 2002, ApJ, 574,430

Wright, E. L., et al. 2010, AJ, 140, 1868

Wright, J. T., \& Gaudi, B. S. 2013, in Planets, Stars and Stellar Systems, Solar and Stellar Planetary Systems, Vol. 3, ed. T. D. Oswalt, L. M. French, \& P. Kalas, (Dordrecht: Springer), 489

Yamamura, I., Tsuji, T., \& Tanabé, T. 2010, ApJ, 722, 682

Yurchenko, S. N., Barber, R. J., \& Tennyson, J. 2012, MNRAS, 413, 1828

Yurchenko, S. N., Tennyson, J., Barber, R. J., \& Thiel, W. 2013, JMoSp, 291, 69

Yurchenko, S. N., \& Tennyson, J. 2014, MNRAS, 440, 1649

Yurchenko, S. N., Tennyson, J., Bailey, J., Hollis, M. D. J., Tinnetti, G. 2014, PNAS, 111, 9379

Zahnle, K., Marley, M. S., Freedman, R., Lodders, K., \& Fortney, J. J. 2009, ApJL, 701, L20

Zahnle, K., \& Marley, M. S. 2014, submitted to ApJ, arXiv: 1408.6283

Zapatero Osorio, M. R., Caballero, J. A., \& Béjar, V. J. S. 2005, ApJ, 621, 445

Zellem, R. T., et al. 2014, ApJ, 790, 53

Zhang, X., \& Showman, A. P. 2014, ApJL, 788, L6

Zhao, M., Monnier, J. D., Swain, M. R., Barman, T., \& Hinkley, S. 2012a, ApJ, 744, 122

Zhao, M., Milburn, J., Barman, T., Hinkley, S., Swain, M. R., Wright, J., \& Monnier, J. D. 2012b, ApJL, 748, L8

Zhou, G., \& Bayliss, D. R. 2012, ApJ, 426, 2483

Zhou, G., Kedziora-Chuczer, L., Bayliss, D. R., \& Bailey, J. 2013, ApJ, 774, 9

Zhou, G., Bayliss, D. D. R., Kedziora-Chudczer, L., Salter, G., Tinney, C. G., \& Bailey, J. 2014, MNRAS, in press, arXiv: 1409.2775

Zsom, A., Seager, S., de Wit, J., \& Stamenković, V. 2013, ApJ, 778, 109

Zugger, M. E., Kasting, J. F., Williams, D. M., Kane, T. J., \& Philbrick, C. R. 2010, ApJ, 723, 1168

Zugger, M. E., Kasting, J. F., Williams, D. M., Kane, T. J., \& Philbrick, C. R. 2011, ApJ, 739, 12 